\title{
The 2005 Study of Organic Aerosols at Riverside (SOAR-1): instrumental intercomparisons and fine particle composition
}

\author{
K. S. Docherty ${ }^{1,2, *}$, A. C. Aiken ${ }^{1,2, * *}$, J. A. Huffman ${ }^{1,2, * * *}$, I. M. Ulbrich ${ }^{1,2}$, P. F. DeCarlo ${ }^{1,3, * * * *}$, D. Sueper ${ }^{1,2,4}$, \\ D. R. Worsnop ${ }^{4}$, D. C. Snyder ${ }^{5, * * * *}$, R. E. Peltier ${ }^{6, * * * * *}$, R. J. Weber ${ }^{6}$, B. D. Grover ${ }^{7}$, D. J. Eatough ${ }^{7}$, \\ B. J. Williams ${ }^{8, * * * * * * *}$, A. H. Goldstein ${ }^{8}$, P. J. Ziemann ${ }^{9}$, and J. L. Jimenez ${ }^{1,2}$ \\ ${ }^{1}$ Cooperative Institute for Research in Environmental Sciences, University of Colorado, Boulder, Colorado, USA \\ ${ }^{2}$ Dept. of Chemistry and Biochemistry, University of Colorado, Boulder, Colorado, USA \\ ${ }^{3}$ Dept. of Oceanic and Atmospheric Science, University of Colorado, Boulder, Colorado, USA \\ ${ }^{4}$ Aerodyne Research, Inc., Billerica, Massachusetts, USA \\ ${ }^{5}$ Environmental Chemistry and Technology Program, University of Wisconsin, Madison, Wisconsin, USA \\ ${ }^{6}$ School of Earth and Atmospheric Sciences, Georgia Institute of Technology, Atlanta, Georgia, USA \\ ${ }^{7}$ Dept. of Chemistry and Biochemistry, Brigham Young University, Provo, Utah, USA \\ ${ }^{8}$ Dept. of Environmental Science, Policy, and Management, University of California, Berkeley, California, USA \\ ${ }^{9}$ Air Pollution Research Center and Depts. of Environmental Science and Chemistry, University of California, Riverside, \\ California, USA \\ *currently at: Alion Science and Technology, US EPA Office of Research and Development, Research Triangle Park, North \\ Carolina, USA \\ ** currently at: Los Alamos National Laboratory, Los Alamos, New Mexico, USA \\ **** currently at: Max Planck Institute for Chemistry, Mainz, Germany \\ ***** currently at: AAAS Science and Technology Policy Fellow hosted by the US EPA, Washington, DC, USA \\ ****** currently at: Department of Chemistry, University of Wisconsin, Stevens Point, Wisconsin, USA \\ ******* currently at: School of Public Health, University of Massachusetts, Amherst, Massachusetts, USA \\ ******** currently at: Department of Energy, Environmental, \& Chemical Engineering, Washington University,
}

St. Louis, Missouri, USA

Received: 2 November 2010 - Published in Atmos. Chem. Phys. Discuss.: 23 February 2011

Revised: 28 October 2011 - Accepted: 2 November 2011 - Published: 12 December 2011

\begin{abstract}
Multiple state-of-the-art instruments sampled ambient aerosol in Riverside, California during the 2005 Study of Organic Aerosols at Riverside (SOAR) to investigate the chemical composition and potential sources of fine particles $\left(\mathrm{PM}_{f}\right)$ in the inland region of Southern California. In this paper, we briefly summarize the spatial, meteorological and gas-phase conditions during SOAR-1 (15 July-15 August), provide detailed intercomparisons of high-resolution aerosol mass spectrometer (HR-AMS) measurements against complementary measurements, and report the average composition of $\mathrm{PM}_{f}$ including the composition of the organic fraction measured by the HR-AMS. Daily meteorology and gas-phase species concentrations were highly consistent, dis-
\end{abstract}

Correspondence to: J. L. Jimenez (jose.jimenez@colorado.edu) playing clear diurnal cycles and weekday/weekend contrast. HR-AMS measurements of non-refractory submicron (NR$\mathrm{PM}_{1}$ ) mass are consistent and highly correlated with those from a filter dynamics measurement system tapered-element oscillating microbalance (TEOM), while the correlation between HR-AMS and heated TEOM measurements is lower due to loss of high volatility species including ammonium nitrate from the heated TEOM. Speciated HR-AMS measurements are also consistent with complementary measurements as well as with measurements from a collocated compact AMS while HR-AMS OC is similar to standard semicontinuous Sunset measurements within the combined uncertainties of both instruments. A correction intended to account for the loss of semi-volatile OC from the Sunset, however, yields measurements $\sim 30 \%$ higher than either HRAMS or standard Sunset measurements. On average, organic aerosol (OA) was the single largest component of $\mathrm{PM}_{f}$. OA

Published by Copernicus Publications on behalf of the European Geosciences Union. 
composition was investigated using both elemental analysis and positive matrix factorization (PMF) of HR-AMS OA spectra. Oxygen is the main heteroatom during SOAR-1, with $\mathrm{O} / \mathrm{C}$ exhibiting a diurnal minimum of 0.28 during the morning rush hour and maximum of 0.42 during the afternoon. $\mathrm{O} / \mathrm{C}$ is broadly anti-correlated with $\mathrm{H} / \mathrm{C}$, while $\mathrm{N} / \mathrm{C}$ and $\mathrm{S} / \mathrm{C}$ (excluding organonitrate $(\mathrm{ON})$ and organosulfate (OS) functionalities) are far lower than $\mathrm{O} / \mathrm{C}$ at about 0.015 and $\sim 0.001$, respectively. When $\mathrm{ON}$ and $\mathrm{OS}$ estimates are included $\mathrm{O} / \mathrm{C}, \mathrm{N} / \mathrm{C}$, and S/C increase by factors of 1.21, 2, and 30 , respectively, while $\mathrm{H} / \mathrm{C}$ changes are insignificant. The increase in these ratios implies that $\mathrm{ON}$ accounts for $\sim 1 / 2$ of the organic nitrogen while OS dominate organic sulfur at this location. Accounting for the estimated ON and OS also improves the agreement between anions and cations measured by HR-AMS by $\sim 8 \%$, while amines have only a very small impact $(1 \%)$ on this balance. Finally, a number of primary and secondary OA components were resolved by PMF. Among these a hydrocarbon-like OA and two minor, local OA components, one of which was associated with amines, were attributed to primary emissions and contributed a minor fraction $(\sim 20 \%)$ of OA mass. The remaining OA mass was attributed to a number of secondary oxidized OA (OOA) components including the previously-identified lowvolatility and semi-volatile OOA components. In addition, we also report for the first time the presence of two additional OOA components.

\section{Introduction}

Aerosols are of interest due to their widespread impact on atmospheric processes including radiative forcing, heterogeneous reactions, visibility reduction on both local and regional scales, and their role in increased human morbidity and mortality. The role of aerosols in each of these processes is influenced both by particle size and chemical composition, with many of the effects being correlated with fine particles $\left(\mathrm{PM}_{f}\right.$, typically defined as particles having aerodynamic diameters, $d_{\mathrm{a}}, \leq 2.5 \mu \mathrm{m}\left(\mathrm{PM}_{2.5}\right)$ or $\left.\leq 1 \mu \mathrm{m}\left(\mathrm{PM}_{1}\right)\right)$. The effect of chemical composition on these processes is not well understood due both to the complexity of $\mathrm{PM}_{f}$ and to its rapid variation in space and time. Generally, $\mathrm{PM}_{f}$ is comprised of mixtures of organic species (collectively "organic aerosols", $\mathrm{OA})$, "black" or "elemental" carbon (BC or EC), and inorganic species (both soluble ionic and crustal). Although the contributions of insoluble inorganic species such as mineral dust and metals are uncertain due primarily to instrumental limitations, the bulk of inorganics resulting from secondary atmospheric processes, including ammonium nitrate and ammonium sulfate, are reasonably well characterized due to the small number of species involved and their relatively simple chemistry.
The composition of OA in $\mathrm{PM}_{f}$ remains poorly characterized despite the fact that it contributes a substantial fraction of the $\mathrm{PM}_{f}$ mass globally (Zhang et al., 2007). OA is comprised of thousands of individual compounds either emitted directly into the atmosphere ("primary" OA, POA) or formed in the atmosphere as a result of gas-to-particle conversion ("secondary" OA, SOA). The distinction between POA and SOA can provide greater insight to regulatory and control strategies that seek to reduce emissions from various sources in order to mitigate the negative consequences of $\mathrm{PM}_{f}$ (de Gouw and Jimenez, 2009).

Historically, the majority of efforts to characterize the composition of bulk OA have relied largely on speciated measurement techniques such as gas chromatography/mass spectrometry (GC/MS). While these techniques have proven successful at identifying and quantifying chemically reduced compounds that typically comprise POA, their characterization of SOA has been challenged by either the low volatility, high degree of oxidation, or labile nature of SOA species. For example, while a distinct set of organic molecular marker (OMM) species have been identified from GC/MS analyses and used to successfully apportion POA among various sources in a bottom-up chemical mass balance (CMB-OMM) approach (e.g., Schauer et al., 1996; Schauer, 2002), molecular markers representing SOA have only recently been isolated using derivatization techniques and applied in CMBOMM models (Kleindienst et al., 2007; Stone et al., 2009). Recent advancements in instrumentation, such as an in-situ thermal desorption aerosol gas chromatograph (TAG), also show promise for further characterizing OA composition based on GC/MS measurements (Williams et al., 2010).

In addition to GC/MS analysis, recent advancements in OA instrumentation have enabled new approaches to its characterization (Hallquist et al., 2009). For example, the aerosol mass spectrometer (AMS) (Jayne et al., 2000; Canagaratna et al., 2007) was developed to monitor the bulk chemical composition of OA in real time and complement speciated analysis techniques. The AMS reports non-refractory submicron $\left(\mathrm{NR}-\mathrm{PM}_{1}\right)$ particle mass in good agreement with collocated instruments at a variety of locations (Canagaratna et al., 2007 and references therein). Factor analysis of AMS OA spectra has additionally allowed for the characterization of several OA components (Zhang et al., 2005a; Lanz et al., 2007; Ulbrich et al., 2009) that are generally consistent at many locations throughout the world (Zhang et al., 2007; Jimenez et al., 2009; Ng et al., 2010, 2011). Despite these recent advancements, however, large uncertainties remain regarding the various sources, formation mechanisms, and composition of ambient OA.

The Study of Organic Aerosols at Riverside (SOAR) was organized to advance our understanding of both the sources and composition of $\mathrm{PM}_{f}$ and OA by deploying in the field for the first time several new instruments with more established instruments as well as to apply the results of advanced source apportionment techniques with those of traditional methods. 
SOAR-1 (15 July-15 August 2005) represents one of the most comprehensive efforts to date to characterize ambient OA composition and sources (Docherty and Jimenez, 2005). Table S1 presents a list of participating groups and instrumentation deployed during SOAR and resulting publications to date. Riverside is located $82 \mathrm{~km}$ east of the urban center of Los Angeles at the Eastern end of the South Coast Air Basin (SoCAB), which is bordered on the north and east by tall mountains ranging in height from $1000 \mathrm{~m}$ and $2900 \mathrm{~m}$ that inhibit the movement of air masses out of the basin. Additionally, during the summer the region has a pronounced sea breeze circulation (westerly) that transports air masses inland from the coast and is characterized by strong temperature inversions that limit vertical dilution of pollutants. Thus, the topography and meteorology of the SoCAB cause Riverside and the surrounding communities to routinely experience very high $\mathrm{PM}_{f}$ concentrations, which consistently rank among the highest in the United States on both shortterm and annual bases (American Lung Association, 2011).

Many of the novel instruments that sampled during SOAR1, including the Aerodyne high-resolution aerosol mass spectrometer (HR-AMS), were first deployed in the field during SOAR-1. As described in DeCarlo et al. (2006), the HRAMS enhances the performance and information content of previous AMS versions and has been adopted very rapidly by many research groups for the characterization of aerosol chemical composition. Of the many benefits of the HR-AMS are a high time resolution (DeCarlo et al., 2006) and an enhanced mass resolution $(m / \Delta m) \sim 4000-5000)$, which allows for elemental analysis (EA, Aiken et al., 2008) of OA spectra. In addition to the HR-AMS, other instruments fielddeployed for the first time in an urban location during SOAR1 included the TAG (Williams et al., 2006; Kreisberg et al., 2009), the aircraft aerosol time-of-flight mass spectrometer (A-ATOFMS) (Pratt et al., 2009b), a dual-oven Sunset monitor (Grover et al., 2009), a particle-into-liquid sampler for total organic carbon (PILS-OC) (Peltier et al., 2007), and a water-based nano-particle counter (Iida et al., 2008). SOAR1 was also the first use of the fast-stepping thermodenuder (TD, Huffman et al., 2008) which was interfaced to both the HR-AMS (Huffman et al., 2009) and the A-ATOFMS (Denkenberger et al., 2007; Pratt and Prather, 2009; Pratt et al., 2009a) to investigate chemically-resolved volatility of ambient aerosols. Many of these instruments have subsequently been used widely in aerosol research and air quality monitoring applications and their respective measurements are commonly used in source apportionment efforts.

The majority of inorganic $\mathrm{PM}_{f}$ mass in the SoCAB is contributed by ammonium, nitrate, and sulfate. Among inorganic anions, particulate nitrate concentrations are enhanced in the vicinity of Riverside (Chow et al., 1994; Kim and Hopke, 2007) by high concentrations of ammonium nitrate. This enhancement is largely driven by gas-to-particle conversion of nitric acid by gaseous ammonia in interior regions of the basin (Neuman et al., 2003). Although sources of ammo- nia in the SoCAB include agricultural activities, emissions from light-duty vehicles, and households, the largest emissions originate from dairy operations. While the number of dairies operating within the basin is smaller than it once was, ammonia emissions remain high and were estimated to still be nearly 18 tons day $^{-1}$ in 2004 (Lester and Woods, 2004). Unlike nitrate, sulfate concentrations are more uniform across most SoCAB locations (Chow et al., 1994; Kim and Hopke, 2007) and are driven by the influx of marine background aerosol and primary emissions of sulfur dioxide from an array of stationary and mobile sources. Among the mobile sources, the most significant are commercial shipping operations centered around the ports of Los Angeles and Long Beach, which contribute $>50 \%$ of total basin-wide $\mathrm{SO}_{2}$ emissions (Eckerle et al., 2007). The impact of sulfate emissions from shipping activities on air quality within the basin is projected to substantially increase in the future $(\mathrm{Vu}-$ tukuru and Dabdub, 2008; Huang et al., 2010).

OA contributes the remainder, and often a majority, of $\mathrm{PM}_{f}$ mass in the inland regions of the SoCAB including Riverside (Chow et al., 1994; Kim et al., 2000; Christoforou et al., 2000). A variety of methods, including selective solvent-extraction-carbon analysis and high resolution mass spectrometry (Appel et al., 1979), regional modeling (Pandis et al., 1992), EC-tracer analysis (Gray et al., 1986; Hildemann et al., 1993), and CMB-OMM (Schauer et al., 1996), have been used to apportion OA mass in the SoCAB between POA and SOA. Collectively, these studies concluded that, outside of short-term photochemical smog episodes, characterized by sustained, very elevated ozone concentrations (Turpin and Huntzicker, 1995; Schauer et al., 2002), the majority of OA is directly emitted POA.

In contrast, several independent source apportionment efforts conducted using measurements acquired during SOAR1 consistently indicated that OA was dominated by SOA. For example, Eatough et al. (2008) applied positive matrix factorization (PMF) to aggregate 1-h semi-continuous measurements from a suite of instruments. The analysis of Eatough et al. (2008) which incorporated HR-AMS and ATOFMS data attributed over three-quarters of total $\mathrm{PM}_{f}$ to secondary processes, with about equal amounts due to local production vs. downwind transport from Los Angeles. Similarly, Williams et al. (2010) applied PMF for the first time to hourly TAG measurements obtained during SOAR. Four SOA components were identified from the analysis of SOAR-1 data that were largely attributed to the oxidation of anthropogenic precursor gases and, when combined, contributed the bulk $(68.5 \%)$ of OA mass, on average, consistent with the dominance of SOA during this period. The most comprehensive source apportionment analysis of SOAR-1 data was performed by Docherty et al. (2008), where several independent source apportionment techniques (EC-tracer, CO-tracer, CMB-OMM, water soluble organic carbon (WSOC), and PMF of HR-AMS OA spectra) were applied to SOAR-1 OA measurements. These five methods consistently indicated 
that, on average, the bulk of OA mass (average $78 \%$ ) was secondary in nature, despite the absence of "photochemical smog episode" conditions during SOAR-1.

A number of underlying factors may have led to the observed difference between historical apportionments of POA and SOA and those obtained using SOAR-1 measurements (Docherty et al., 2008). One possibility is that deficiencies in or problems with the methods applied in previous apportionment efforts underreported the contribution of SOA due to difficultiles inherent in its measurement. A second possibility is the success of regulatory strategies targeted at reducing POA emissions (e.g., via cleaner burning diesel fuel and diesel engines) relative to the reduction of SOA precursors, which may have effectively increased the fraction of SOA in the SoCAB as a result. Due to current uncertainties in the dominant precursors and pathways of SOA formation in urban areas, it is difficult to conclusively identify the impact of air quality improvement policies or determine those factors responsible for this discrepancy.

Beyond the overwhelming contribution of SOA during SOAR-1, many publications resulting from the study have indicated the unique natue of $\mathrm{OA}$ in the SoCAB basin. For example, Stone et al. (2009) applied CMB-OMM to filter samples collected in Riverside during SOAR-1 and a number of midwestern United States locations using both traditional POA tracers and a number of SOA tracers identified from chamber photooxidation of isoprene, $\alpha$-pinene, $\beta$ caryophylene, and toluene. In Riverside, only one-quarter of OA mass could be attributed to POA sources, while a similar amount could be attributed to known secondary sources. The remaining $50 \%$ of the measured OA (significantly higher than the unattributed fraction from Midwest locations) could be attributed to neither primary nor secondary sources. This fraction was expected to be secondary in nature based on both the agreement of the POA apportionment with those of multiple other methods (Docherty et al., 2008; Williams et al., 2010) and its chemical characteristics. These results indicate the presence of additional sources and mechanisms of SOA formation in the SoCAB beyond those considered in the Stone et al. (2009) study, namely aromatic and biogenic precursors.

Investigations into the composition of the water soluble OA fraction during SOAR-1 have also indicated the presence of unique compounds attributed to SOA production. For example, Reemtsma et al. (2006) observed several classes of compounds, including fulvic acids and high molecular weight sulfur-, nitrogen-, and sulfur- and -nitrogencontaining compounds using offline electrospray ionization ultrahigh resolution mass spectrometry of the water soluble OA fraction. Such analogues have not previously been identified from terrestrial humic-like substances (HULIS) and were attributed to SOA formation. Previous investigations have identified organosulfates (OS) from chamber reactions (Surratt et al., 2007) and in ambient OA in rural areas (Surratt et al., 2008; Lukacs et al., 2009). Their contribution to urban
OA, however, is largely unknown. Stone et al. (2009) investigated the contribution of functional groups to the water soluble OA fraction in a variety of locations including Riverside using liquid chromatography tandem mass spectrometry to better understand the formation of HULIS in atmospheric aerosols. Results of this analysis suggest that motor vehicles, biomass burning, and SOA each may make significant contributions to HULIS formation. Additionally, OS in the range $m / z$ 200-600 made non-negligible contributions (0.6$3.2 \%$ of total measured carbon) to OA in Riverside, indicating both an appreciable contribution of OS during SOAR and a potential role for oligomers in the formation and growth of OA within the SoCAB.

Oligomers have been frequently identified from OA formed in chamber reactions (Kalberer et al., 2004; Tolocka et al., 2004; Gao et al., 2004), but have not been widely identified in ambient aerosols due to their tendency to thermally decompose back to the constituent monomers when subjected to traditional analytical techniques such as GC/MS (Tolocka et al., 2004). Denkenberger et al. (2007), however, observed signature oligomeric spectra during SOAR1 using the A-ATOFMS. Oligomer concentrations were observed to be highest in small particles $\left(d_{\mathrm{va}}=140-200 \mathrm{~nm}\right)$ and occurred coincident with markers including oxidized $\mathrm{OA}$, amines, nitrate, and sulfate indicating their formation through atmospheric aging. Oligomer signatures were found in both unheated and heated aerosols when the A-ATOFMS was interfaced with the TD. However, the relative intensity of oligomeric spectra increased at high TD temperatures $\left(>150^{\circ} \mathrm{C}\right.$ ) suggesting that either the oligomeric fraction of ambient OA has a relatively low volatility or that oligomer formation is accelerated in the TD at high temperatures due to the removal of semi-volatile species or increased acidity (Denkenberger et al., 2007). Oligomeric spectra were not directly observed by the HR-AMS. However, in analyzing thermally-denuded HR-AMS data, Huffman et al. (2009) observed an OA fraction with very low volatility. Although TD measurements have consistently indicated that NR-PM sulfate (mostly as ammonium sulfate) has a lower average volatility than bulk OA, a small fraction of OA dominated the residual mass at the highest TD temperatures in both Riverside and Mexico City, consistent with the potential presence of oligomers in aerosol in these locations that may be formed or enhanced due to TD heating.

Given the collocation of a wide variety of aerosol instruments, SOAR-1 also provides an opportunity to evaluate the performance of novel instruments against those that are more widely employed in the field and to evaluate the consistency of various measurements in order to identify the existence of any potential biases on the part of either instrument. Although a number of SOAR-1 publications have previously compared complementary measurements from different instruments and found them to be consistent, these comparisons were limited in scope with respect to the number of corresponding measurements included in the comparison or the 
duration of the comparison, particularly with respect to OA measurements. Grover et al. (2008) for example evaluated mass closure using a complement of $\mathrm{PM}_{2.5}$ measurements from several instruments and found that they were consistent and highly correlated, although poor correlation was observed among the various sulfate measurements. With respect to OA measurements, Grover et al. (2008) compared only a limited number (30) of 1-h averaged total carbon measurements from the dual-channel Sunset and the Particle Concentrator-Brigham Young University Organic Sampling System (PC-BOSS) and found measured concentrations from both instruments to be consistent and well correlated.

Both Grover et al. (2009) and Snyder and Schauer (2007) compared EC measurements from the dual-channel and standard Sunset instruments, respectively, with BC measured by collocated instruments. Although measurements from the various instruments were consistent and highly correlated, similar intensive comparisons of OC measurements during SOAR-1 have not been presented. The most comprehensive comparison of OC is that of Peltier et al. (2007) who compared PILS-OC and Sunset OC measurements over a period of three days. Although PILS-OC and Sunset measurements were highly correlated, an intercept of -1.69 indicated either a positive offset for the Sunset instrument or an inability of the PILS-OC instrument to analyze larger insoluble particles (Peltier et al., 2007). These results clearly demonstrate the intrinsic value of comparing complementary measurements in order to understand the limitations of both novel and widely accepted measurement techniques, particularly over long periods.

In order to capitalize on the collocation of many stateof-the-art aerosol instruments and expand instrumental comparisons to a larger set of measurements under conditions where SOA dominated the composition of OA, here we provide additional detailed comparisons among measurements obtained by the HR-AMS and an ensemble of $\mathrm{PM}_{2.5}$ instruments that are routinely employed in the characterization of ambient aerosol composition. Additionally, to establish a unified framework for SOAR publications and other studies in the LA area (such as CalNex-2010) we report the average composition of $\mathrm{PM}_{f}$ measured by both the HR-AMS and the ensemble of $\mathrm{PM}_{2.5}$ instruments. Finally, we report results of elemental analysis and positive matrix factorization of HRAMS OA.

\section{The Study of Organic Aerosols at Riverside (SOAR)}

\subsection{Representativeness of the sampling site}

The sampling site was located near potential local emission sources, including the I-215 highway, a major interstate highway that carries $\sim 170000$ automobiles per day in the vicinity of UC-Riverside (CalTrans, 2010), located $\sim 0.5 \mathrm{~km}$ west of the site. Maps of SoCAB topography and total pollutant emissions are provided in Fig. S8 for reference. During the day, the prevailing winds typically arrive to the site from the west, placing the site downwind of the interstate.

The goal of SOAR-1 was to characterize the composition of $\mathrm{PM}_{f}$ in the Eastern LA basin. To evaluate the representativeness of the site and the extent to which local sources such as this major highway contributed to $\mathrm{PM}_{f}$ we compare on- and off-site $\mathrm{PM}_{2.5}$ measurements. Off-site measurements were obtained from a beta-attenuation monitor (BAM) operated at the California South Coast Air Quality Management District, Rubidoux monitoring site located $\sim 7 \mathrm{~km}$ west of the SOAR-1 sampling site. The Rubidoux site is upwind of I-215 and other major interstates during daytime hours when traffic is heaviest. Rubidoux BAM measurements are compared with those of a tapered element oscillating microbalance with a filter dynamics measurement system (TEOM FDMS $_{\text {) }}$ (Patashnik et al., 2001) located at the SOAR-1 site as these two techniques have been shown to provide equivalent $\mathrm{PM}_{2.5}$ measurements (Schwab et al., 2006; Grover et al., 2006).

TEOM $_{\text {FDMS }}$ and Rubidoux BAM measurements during SOAR-1 are compared in Fig. 1. Overall, the measurements

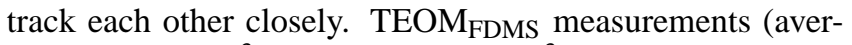
age $=28.6 \mu \mathrm{g} \mathrm{m}^{-3}$; range $=9-68 \mu \mathrm{g} \mathrm{m}^{-3}$ ) are similar to those from Rubidoux BAM $\left(31.0 \mu \mathrm{g} \mathrm{m}^{-3} ; 6-76 \mu \mathrm{g} \mathrm{m}^{-3}\right)$ and are well correlated $\left(r^{2}=0.62\right)$ despite the geographical separation of the two instruments. BAM measurements are consistently higher during overnight and morning hours, possibly due to an increased influence of motor vehicles near Rubidoux. Linear regression yields a slope of 0.87 with a recovery slope (i.e., when a fixed intercept of zero is used) of 0.91 . The similarity and strong correlation of mass concentrations measured at both locations highlight the spatial consistency of $\mathrm{PM}_{f}$ mass in inland regions of the SoCAB. This also suggests that the contribution of $\mathrm{PM}_{f}$ mass from sources within a few miles of the SOAR-1 sampling site is a minor fraction of the measured concentrations. This is consistent with the smooth variations in the time series of most species described below and in previous publications. Although their mass contributions are minor, these sources, particularly traffic along I-215, emit large numbers of ultrafine particles which significantly impact number concentrations at the site (Cubison et al., 2008).

\subsection{Meteorology, gas-phase pollutant concentrations, and lack of biomass burning events during SOAR-1}

Meteorological conditions during SOAR-1 were typical for SoCAB inland valleys during the summer and exhibited little day-to-day variation. Time series and diurnal profiles of meteorological variables and gas-phase species during SOAR-1 (Fig. S9) contrast with typical wintertime conditions during SOAR-2 (15 October-15 November, Fig. S10). During SOAR-1, relative humidity $(\mathrm{RH})$ and temperature were generally anti-correlated with an average maximum 

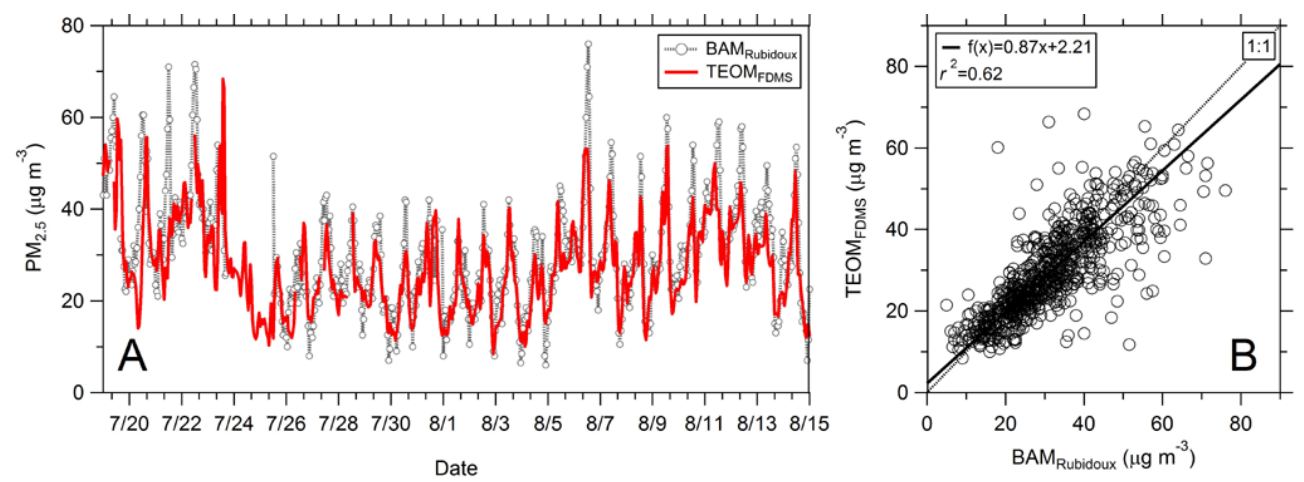

Fig. 1. Comparison of on- and off-site $\mathrm{PM}_{2.5}$ mass concentrations: (a) time series and (b) scatter plot. On-site measurements were obtained from the TEOM $\mathrm{FDMS}$ while off-site measurements were obtained by the Rubidoux BAM.

$(\sim 80 \%)$ coinciding with average minimum temperatures $\left(\sim 20^{\circ} \mathrm{C}\right)$ during the early morning hours while average minimum $(\sim 30 \%)$ coincided with average maximum temperature $\left(\sim 35^{\circ} \mathrm{C}\right)$ during mid-day. Absolute humidity (shown as the dew point in Fig. S9) showed little variation during most of SOAR-1. Inland regions of the SoCAB, including Riverside, were characterized by a prominent sea breeze circulation during SOAR-1. Nighttime wind speeds were low $\left(\sim 0.2 \mathrm{~m} \mathrm{~s}^{-1}\right)$ with variable, generally southerly, direction while maximum speed $\left(\sim 2 \mathrm{~m} \mathrm{~s}^{-1}\right)$ westerly winds were experienced during the warmest part of the day.

Average ozone $\left(\mathrm{O}_{3}\right)$ and odd oxygen $\left(\mathrm{O}_{\mathrm{x}}\right)$ concentrations (Fig. S9d) were lowest during overnight periods with maximum average concentrations of $\sim 90$ and $\sim 100$ ppbv, respectively, observed just after noon. Primary combustion emission markers including carbon monoxide $(\mathrm{CO})$, oxides of nitrogen $\left(\mathrm{NO}_{\mathrm{x}}\right)$, and EC (Fig. S9e) displayed the opposite trend, with elevated concentrations throughout the overnight period and maximum average concentrations of $\sim 600 \mathrm{ppb}, \sim 60 \mathrm{ppb}$, and $2 \mu \mathrm{g} \mathrm{m}^{-3}$, respectively, during the early morning, coincident with morning rush hour traffic. The concentrations of these primary species declined rapidly to their minima during the afternoon and early evening periods, likely due to the combined effects of decreased emissions, increased vertical dilution from the growing boundary layer, and perhaps also horizontal advection.

Although the SoCAB is often severely impacted by wildfires that can significantly increase particle concentrations (Phuleria et al., 2005), the contribution of biomass burning OA (BBOA) during SOAR-1 was minimal according to many different metrics (Docherty et al., 2008), including the MODIS Active Fire Detections database (http://maps.geog. umd.edu/firms/), which confirmed the low incidence of wildfires in and around the SoCAB. Low BBOA contributions during SOAR-1 were confirmed by ground-based measurements at the site, including background levels of the ratio of HR-AMS $\mathrm{m} / \mathrm{z}, 60$ to OA signal (Aiken et al., 2009, 2010; Lee et al., 2010) and measurements of the molecu- lar marker levoglucosan that indicated small BBOA contributions (Docherty et al., 2008). When biomass burning particles were observed, they were highly aged indicating their having been transported long distances to the site (Pratt and Prather, 2009).

\section{Experimental}

\subsection{General considerations}

Sampling was conducted at the Air Pollution Research Center on the University of California-Riverside (UC-Riverside) campus $\left(33^{\circ} 58^{\prime} 18.40^{\prime \prime} \mathrm{N}, 117^{\circ} 19^{\prime} 21.41^{\prime \prime} \mathrm{W}, \sim 210 \mathrm{~m}\right.$ elevation). All times herein refer to Pacific Standard Time (PST, i.e., local time $-1 \mathrm{~h}$.) and all concentrations are reported at ambient temperature and pressure. Unless otherwise stated, all linear regressions were performed in Igor Pro (Wavemetrics, Lake Oswego, OR, USA) using an orthogonal distance regression technique which is appropriate for fitting data when there are measurement errors in both variables.

\subsection{PM $_{2.5}$ measurements}

Hourly $\mathrm{PM}_{2.5}$ mass was measured by two Rupprecht and Patashnick (R\&P, Thermo Fisher Scientific, Waltham, MA, USA) tapered element oscillating microbalances (TEOM). A heated TEOM $\left(\right.$ TEOM $\left._{50 \mathrm{C}}\right)$ was operated with the inlet and instrument filter at $50^{\circ} \mathrm{C}$ to remove particle-bound water and water adsorbed to the filter, which can cause high measurement noise. The elevated temperature results in collateral loss of semi-volatile material (SVM), including ammonium nitrate $\left(\mathrm{NH}_{4} \mathrm{NO}_{3}\right)$ and semi-volatile organic material (SVOM) (Eatough et al., 2003; Patashnik et al., 2001). As a result, the TEOM ${ }_{50 \mathrm{C}}$ measurement is considered a measure of less-volatile $\mathrm{PM}_{2.5}$ mass. In contrast, the TEOM $\mathrm{FDMS}_{\mathrm{F}}$ uses intermittent sampling through a HEPA filter to account for volatilized SVM mass. Therefore, this method is considered a comprehensive measurement of both non-volatile and 
semi-volatile $\mathrm{PM}_{2.5}$ mass (Grover et al., 2008). $\mathrm{PM}_{2.5}$ inorganic nitrate (IC-nitrate) and sulfate (IC-sulfate) concentrations were also measured hourly by a Dionex (Thermo Fisher Scientific, Waltham, MA, USA) GP-IC ion chromatography system (Grover et al., 2008). This system samples ambient aerosol through a parallel plate wet denuder using $0.5 \mathrm{mM}$ hydrogen peroxide as a scrubber liquid to remove soluble inorganic gases while water is condensed on remaining particles. Water is passed through a $0.5 \mathrm{~mm}$ filter prior to analysis to remove insoluble particles. The solution on the downstream side of the filter is aspirated by a peristaltic pump and sent to a Dionex TAC-ULP1 preconcentration column of an ion chromatograph for anion analysis.

Hourly $\mathrm{PM}_{2.5}$ OC and EC were measured using both a standard (Sunset1, Snyder and Schauer, 2007) and a dualoven (Sunset2, Grover et al., 2008) semi-continuous carbon monitor (Sunset Laboratories, Tigard, OR, USA) using the NIOSH 5040 method (Bae et al., 2004). Briefly, Sunset1 sample collection was accomplished at a flow rate of $24.01 \mathrm{~min}^{-1}$ through an inlet equipped with a sharp-cut $\mathrm{PM}_{2.5}$ cyclone and a carbon-impregnated parallel-plate denuder designed to remove gas-phase organic compounds upstream of the collection filter. In contrast, the Sunset2 sampled at $81 \mathrm{~min}^{-1}$ through a $\mathrm{PM}_{2.5}$ sharp-cut cyclone and a similar parallel-plate charcoal-impregnated-filter denuder, both of which were supplied by the manufacturer. Sunset $1 \mathrm{EC}$ concentrations compared well with $\mathrm{BC}$ from two absorption-based instruments and integrated filter EC measurements (Snyder and Schauer, 2007). While Sunset1 concentrations were slightly higher $(\sim 11 \%)$ than comparable filter measurements, they were highly correlated with each set of comparable measurements.

\subsection{NR-PM measurements $_{1}$}

Total and speciated non-refractory submicron $\left(\mathrm{NR}-\mathrm{PM}_{1}\right)$ aerosol measurements were made by a pair of Aerodyne time-of-flight aerosol mass spectrometers (ToF-AMS generally): a high-resolution (HR-AMS, sampling period: 7/148/13/2005) and a unit-mass resolution compact (C-AMS, sampling period: 8/3-8/13/2005) ToF-AMS. Detailed descriptions of these instruments are provided elsewhere (DeCarlo et al., 2006; Drewnick et al., 2005). Ambient air was sampled by both instruments from a height of $\sim 6 \mathrm{~m}$ above ground level through a $\mathrm{PM}_{2.5}$ cyclone (URG Corp., Chapel Hill, NC, USA) at a rate of $\sim 121 \mathrm{~min}^{-1}$. The sampled air was drawn through copper tubing with total transit times of $10-15 \mathrm{~s}$ from the inlet to the ToF-AMS instruments. Unless otherwise stated, ToF-AMS ensemble mass spectra (MS) and major NR-PM 1 component (i.e., ammonium $\left(\mathrm{NH}_{4}\right)$, chloride $(\mathrm{Cl})$, nitrate $\left(\mathrm{NO}_{3}\right), \mathrm{OA}$, and sulfate $\left.\left(\mathrm{SO}_{4}\right)\right)$ concentrations were averaged every five min; ambient sampling was interrupted only for instrument calibrations, maintenance, or to sample from an indoor environmental smog chamber (Aiken et al., 2008; Mohr et al., 2009). Note that we do not use the ion charges in the discussion below when referring to nominally inorganic species (e.g., we use $\mathrm{SO}_{4}$ instead of $\mathrm{SO}_{4}^{2-}$ ) because the ToF-AMS data are known to contain organic contributions, e.g. an OS contribution to the $\mathrm{SO}_{4}^{2-}$ signal, as discussed below.

Unique to SOAR-1 is the fact that both ToF-AMS were operated using specialized sampling protocols including the use of the TD. Details regarding TD design and operation during SOAR-1 are provided in Huffman et al. (2008, 2009), respectively. Briefly, when interfaced with the TD, the sample flow to the ToF-AMS is alternated between ambient (i.e., bypassing the TD) and thermally denuded sampling every 10 min using a set of computer-controlled switching valves. On a longer time scale, the temperature of the TD is cycled through a range of preset temperatures from ambient to $\sim 230^{\circ} \mathrm{C}$. NR-PM 1 mass measured during thermallydenuded sampling periods is lower than during ambient sampling periods due to the removal of a variable mass fraction depending on TD temperature. The alternating sampling modes result in a characteristic saw-tooth time series of ToF-AMS species concentrations. For all analyses discussed herein, except for PMF analysis (ref. Sect. 4.6), thermallydenuded data have been removed, and only routine ambient sampling measurements from both ToF-AMS datasets are compared.

Analysis of unit mass resolution (UMR) ToF-AMS data was conducted using the custom-developed ("Squirrel") software package (Sueper, 2009) which implements the analysis algorithms described previously (Jimenez et al., 2003; Allan et al., 2003, 2004; DeCarlo et al., 2006). A collection efficiency of 0.5 was used for all species in both instruments, typical of aerosols measured in urban locations with similar compositions (e.g., Canagaratna et al., 2007 and references therein) and verified with intercomparisons with collocated instruments (see discussion below). A potentially higher collection efficiency due to high nitrate fraction (Middlebrook et al., 2012) was evaluated and found to increase concentrations by only a few percent. Since this change is much lower than the uncertainty of ToF-AMS measurements, it has not been implemented here. High-resolution (HR) spectra from the HR-AMS were analyzed using a custom data analysis module ("Pika") developed by the Jimenez group (DeCarlo et al., 2006) in Igor Pro. EA of HR OA spectra was conducted according to the procedures of Aiken et al. (2008).

A limited number of spikes present in the HR-AMS OA concentrations during overnight periods were selectively removed during data analysis. These spikes were observed only during a few nights when wind speeds were low and direction was variable, and the spikes were short in duration (5-10 min). OA concentrations more than doubled during a spike, and OA spike mass spectra were similar to those of reduced hydrocarbons such as lubricating oil. Spikes were attributed to emissions from an air compressor that was located at ground level about $8 \mathrm{~m}$ from the ToF-AMS common inlet and operated only at night during the first two weeks of 
SOAR-1 and was then turned off. Spikes were not observed after the use of the compressor was discontinued.

\subsection{PMF of HR-AMS OA mass spectra}

PMF is a receptor-based factorization model which deconvolves complex data using a weighted least squares method (Paatero and Tapper, 1994). The use of PMF to deconvolve AMS OA mass spectra has been discussed in detail elsewhere (Ulbrich et al., 2009). PMF was applied to HR-AMS OA mass spectra obtained between 7/28/2005-8/10/2005. During this period, the HR-AMS was interfaced with the TD to investigate the volatility of the bulk aerosol and its constituents (Huffman et al., 2009). Two separate PMF analyses were conducted using the same nominal dataset to investigate whether TD cycling and the resulting increased variability in OA composition leads to improved resolution of various OA components. In the first (AMS-PMF) analysis, OA spectra collected only during routine ambient sampling (i.e., bypassing the TD) were isolated by excising the thermallydenuded sampling periods. In contrast, a second, separate PMF analysis (TD-AMS-PMF) analyzed combined ambient and thermally-denuded OA spectra. Additional details regarding AMS-PMF and TD-AMS-PMF solutions, their intercomparison, and the choice of a final solution are provided in the Supporting Information.

In each PMF analysis, OA spectra $(\mathrm{m} / z, 12-340)$ were analyzed by combining high resolution (HR, $m / z, 12-100)$ and UMR (m/z 101-340) spectra. HR OA spectra were obtained by removing inorganic (e.g., $\mathrm{N}_{\mathrm{x}} \mathrm{H}_{\mathrm{y}}, \mathrm{N}_{\mathrm{x}} \mathrm{O}_{\mathrm{y}}, \mathrm{S}_{\mathrm{x}} \mathrm{O}_{\mathrm{y}}$ ) and other (e.g., $\mathrm{H}_{\mathrm{x}} \mathrm{O}_{\mathrm{y}}$ ) ions from the total $\mathrm{HR}$ spectrum obtained from the Pika program. UMR OA spectra were calculated with the Squirrel software, which uses the fragmentation tables of Allan et al. (2004). Error matrices for both HR and UMR fragments were calculated using the method of Allan et al. (2003). To equally distribute the weight of HR and UMR spectra, each UMR signal and error value was scaled by the corresponding ratio of $\mathrm{CO}_{2}^{+} / \mathrm{m} / z$ 44. Finally, weak variables $\left(\mathrm{m} / \mathrm{z}^{\prime} \mathrm{s}\right)(0.2<\mathrm{S} / \mathrm{N}<2)$ were downweighted by increasing their calculated error by a factor of three according to the recommendations of Paatero and Hopke (2003). In each case, PMF2.exe (version 4.2) was run in robust mode via a custom panel written for Igor Pro (Ulbrich et al., 2009). Analyses were conducted both with and without imposing an additional PMF model error with little to no change in either time series or mass spectrum of resolved factors. Here, we present results of the analysis using a model error of zero.

\subsection{Gas phase and meteorological measurements}

$\mathrm{CO}, \mathrm{O}_{3}$, and $\mathrm{NO}_{\mathrm{x}}$ concentrations were also measured on-site during SOAR-1. Ambient $\mathrm{CO}$ and $\mathrm{O}_{3}$ were measured every five min by a Thermo Fisher (Thermo Fisher Scientific Inc., Waltham, MA, USA) model 48C nondispersive infrared absorption monitor and a Dasibi (Dasibi Inc., Glendale, CA,
USA) model 1008-RS UV photometric $\mathrm{O}_{3}$ monitor, respectively. $\mathrm{NO}_{\mathrm{x}}$ concentrations were measured every five min by a model $42 \mathrm{C}$ chemiluminescent analyzer (Thermo Fisher Scientific Inc., Waltham, MA, USA) $\mathrm{NO}-\mathrm{NO}_{2}-\mathrm{NO}_{\mathrm{x}}$ monitor. Meteorological conditions were also routinely measured at the SOAR-1 sampling site and are summarized in Fig. S9. Measurements of wind speed and direction were measured every five minutes using an R. M. Young propeller wind monitor (R. M. Young Co., Traverse City, MI, USA).

\section{Results and discussion}

\subsection{Total $\mathrm{PM}_{f}$ intercomparisons}

The TEOM $50 \mathrm{C}$, TEOM ${ }_{\text {FDMS }}$, and HR-AMS measured $\mathrm{PM}_{f}$ mass throughout the duration of SOAR-1. As discussed above, the TEOMs and ToF-AMS differ both in effective size cut, the measurement of refractory material, and the measurement of SVM. Both TEOM instruments sampled through a $\mathrm{PM}_{2.5}$ cyclone (Grover et al., 2008) and their reported concentrations include any refractory material contained within this size fraction. Of the two instruments,

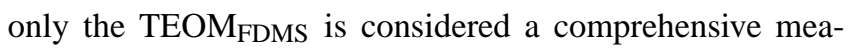
sure of $\mathrm{PM}_{2.5}$ mass. As previously stated, the $\mathrm{TEOM}_{50 \mathrm{C}}$ suffers from evaporation of semi-volatile species including $\mathrm{NH}_{4} \mathrm{NO}_{3}$ and SVOM (Eatough et al., 2003; Patashnik et al., 2001). The difference between the TEOM FDMS $_{\text {S }}$ and $\mathrm{TEOM}_{50 \mathrm{C}}$ has accordingly been used to estimate SVM (Grover et al., 2008). In contrast, the ToF-AMS measures total non-refractory submicron (NR-PM 1 ) mass (e.g., Canagaratna et al., 2007 and references therein). The ToF-AMS does not measure "refractory" materials such as dust, sea salt, and BC as they do not volatilize sufficiently fast at the standard vaporizer temperature and high vacuum conditions to be detected (Canagaratna et al., 2007). As a re-

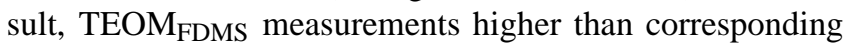
ToF-AMS measurements may be due to (1) non-refractory material between $\mathrm{PM}_{2.5}$ and $\mathrm{PM}_{1}\left(\mathrm{NR}-\mathrm{PM}_{2.5-1}\right)$, (2) $\mathrm{PM}_{2.5}$ refractory material, or a combination thereof. Depending on the actual composition and volatility of the SVM, ToF-AMS measurements can either be larger (e.g., high volatility of SVM) or smaller (e.g., low volatility of SVM) than those obtained by the TEOM $50 \mathrm{C}$. In order to be comparable with the TEOM $_{\text {FDMS }}$, NR-PM 1 must be supplemented by the sum of refractory material in $\mathrm{PM}_{2.5}$ (e.g., $\mathrm{EC}$ and non-EC refractory material such as dust, sea salt, and metals) and NR-PM $2.5-1$. However, because only EC was separately quantified during SOAR-1, we compare the sum of HR-AMS NR-PM ${ }_{1}$ and Sunset1 EC (HR-AMS + EC) with available TEOM measurements.

Although non-EC refractory material was not measured during SOAR-1, an estimate is available from average measurements of $\mathrm{PM}_{2.5}$ aged sea salt and dust. Qin et al. (2011) used ATOFMS measurements to estimate an 

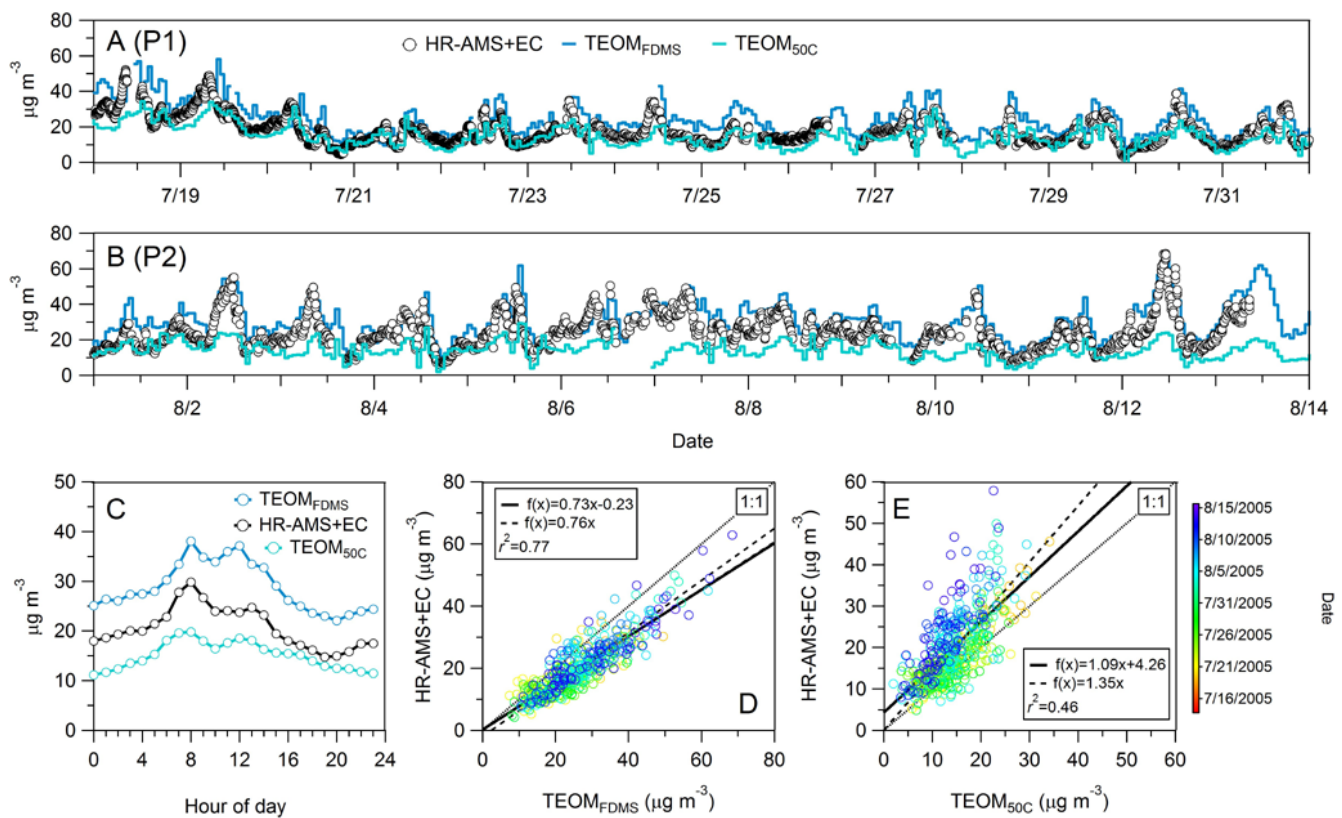

Fig. 2. Comparison of HR-AMS + EC, TEOM ${ }_{F D M S}$, and $\mathrm{TEOM}_{50 \mathrm{C}}$. Time series of each measurement are shown in panels $(\mathbf{A})$ and $(\mathbf{B})$ over the periods 7/17-7/31 and 8/1-8/13, respectively, while panel (C) shows average diurnal profiles of HR-AMS + EC, TEOMFDMS, and $\mathrm{TEOM}_{50 \mathrm{C}}$ throughout the duration of SOAR-1. HR-AMS + EC concentrations are plotted against TEOM $_{\mathrm{FDMS}}$ and the TEOM $50 \mathrm{C}$ concentrations in panels (D) and (E), along with the results of linear regression, with and without a fixed-zero intercept, and coefficients of correlation $\left(r^{2}\right)$.

average concentration of sea salt and dust of $4.7 \mu \mathrm{g} \mathrm{m}^{-3}$, which is consistent with previous measurements in the vicinity of Riverside. For example, measurements by Christoforou et al. (2000) in Rubidoux in 1982, 1986, and 1993 consistently indicated that $\mathrm{PM}_{2.5}$ non-EC refractory material contributes $3-4 \mu \mathrm{g} \mathrm{m}^{-3}$ throughout the year. Similarly, Chow et al. (1994) used measured concentrations of aluminum, silicon, calcium, and iron to obtain an estimate of $1.6 \mu \mathrm{g} \mathrm{m}^{-3}$ for crustal material in Rubidoux during the summer. This latter estimate did not consider contributions from sea salt and is, therefore, likely be a lower bound estimate of non-EC refractory material. More recently, Kim and Hopke (2007) reported a non-EC refractory material estimate of $\sim 3.6 \mu \mathrm{g} \mathrm{m}^{-3}$ in Rubidoux.

TEOM $_{\text {FDMS }}$, TEOM $50 \mathrm{C}$, and HR-AMS + EC time series (TS) are shown in Fig. 2, together with the diurnal profile of each measurement. HR-AMS + EC tracks TEOM ${ }_{\text {FDMS }}$ measurements closely throughout SOAR-1. As expected, average HR-AMS + EC concentrations (average $=19.9 \mu \mathrm{g} \mathrm{m}^{-3}$; range $=4.2-62.9 \mu \mathrm{g} \mathrm{m}^{-3}$ ) are lower than those of the TEOM due to contributions from non-EC refractory and NR$\mathrm{PM}_{2.5-1}$ mass. Diurnal profiles of each measurement (shown in Fig. 2c) are also similar, with prominent maxima at $\sim 08: 00$, concurrent with morning rush-hour traffic, and a second smaller maximum in the early afternoon. As expected from their similar diurnal profiles, the absolute dif- ference between HR-AMS + EC and TEOMFDMS measurements is nearly constant throughout the day. The scatter plot of HR-AMS + EC versus TEOM FDMS (Fig. 2d) shows that HR-AMS + EC and TEOM FDMS are well correlated with an $r^{2}=0.77$, a slope of 0.73 with a small intercept $(0.8 \%$ of

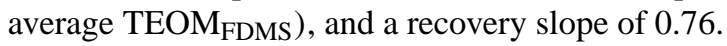

As a result of SVM loss, the 24-h TEOM $50 \mathrm{C}$ average concentration $\left(15.1 \mu \mathrm{g} \mathrm{m}^{-3} ; 2.0-39.7 \mu \mathrm{g} \mathrm{m}^{-3}\right)$ is lower than corresponding TEOM FDMS and HR-AMS + EC values. This is consistent with significant SVM contributions to $\mathrm{PM}_{f}$ previously reported in Riverside (Grover et al., 2005, 2008) and other locations (Cheng et al., 2009; Eatough et al., 2003; Wilson et al., 2006). Interestingly, the contribution of SVM varies during SOAR-1 throughout the day as well as over extended periods. As shown by the diurnal profiles in Fig. 2c, the similarity between TEOM ${ }_{\mathrm{FDMS}}$, HR-AMS + EC, and TEOM $_{50 \mathrm{C}}$ measurements is highest during the late afternoon indicating a low SVM contribution at this time. Daily minimum concentrations are slightly different among the three measurements. While TEOM $\mathrm{FDMS}$ and HR-AMS + EC concentrations reach a minimum during the early evening, $\mathrm{TEOM}_{50 \mathrm{C}}$ concentrations are lowest overnight. Moreover, the contribution of SVM is substantially higher during P2 $(8 / 2-8 / 14)$ as indicated by the larger difference between $\mathrm{TEOM}_{\mathrm{FDMS}}$ and $\mathrm{TEOM}_{50 \mathrm{C}}$ measurements (Table S2). This general trend can also be observed in TS shown in Fig 2a and $b$. A plot of HR-AMS + EC versus TEOM $_{50 C}$ is shown 
in Fig. 2e. The slope in this case is near or higher than unity depending on the choice of intercept. However, these results are likely influenced by the much lower correlation $\left(r^{2}=0.46\right)$ between the measurements caused by the loss of SVM from the TEOM $50 \mathrm{C}$.

A majority of SVM measured during SOAR-1 is $\mathrm{NH}_{4} \mathrm{NO}_{3}$. As shown in Fig. S11, the agreement between TEOM $_{50 \mathrm{C}}$ and TEOM $\mathrm{FDMS}_{\mathrm{F}}$ measurements during both $\mathrm{P}_{1}$ and $\mathrm{P}_{2}$ improves substantially when $\mathrm{TEOM}_{50 \mathrm{C}}$ measurements are supplemented with $\mathrm{PM}_{2.5} \mathrm{NH}_{4} \mathrm{NO}_{3}$ concentrations $\left(\mathrm{TEOM}_{50 \mathrm{C}}+\mathrm{NH}_{4} \mathrm{NO}_{3}\right)$ calculated using IC-nitrate measurements and assuming full neutralization by $\mathrm{NH}_{4}$ (Sect. 4.9). Linear regression of TEOM $50 \mathrm{C}$ against TEOM $\mathrm{FDMS}_{\text {during }}$ $\mathrm{P}_{1}$ and $\mathrm{P}_{2}$ (Fig. S11a) results in slopes of 0.61 and 0.42 , respectively. The value of the slope obtained by linear regression of $\mathrm{TEOM}_{50 \mathrm{C}}+\mathrm{NH}_{4} \mathrm{NO}_{3}$ versus TEOM $\mathrm{FDMS}$ measurements (Fig. S11b) is consistently higher during both periods. However, the increase in slope obtained during $\mathrm{P}_{2}$ is nearly double that during $\mathrm{P}_{1}$, indicating a much higher contribution of $\mathrm{NH}_{3} \mathrm{NO}_{4}$ during the latter sampling period. Assuming that the difference between TEOM $+\mathrm{NH}_{4} \mathrm{NO}_{3}$ were due to volatilization of $\mathrm{SVOM}$, this comparison suggests that SVOM contributions are relatively constant at approximately $24 \%(+2 \%)$ of total $\mathrm{PM}_{2.5}$ mass during both $\mathrm{P}_{1}$ and $\mathrm{P}_{2}$, consistent with the findings of Grover et al. (2008) using measurements of Sunset2.

\subsection{Intercomparison of ToF-AMS measurements}

Previous intercomparisons between two quadrupole AMS (Q-AMS) instruments (Salcedo et al., 2005) and between a Q-AMS and C-AMS (Hings et al., 2007) have shown that collocated AMS instruments generally provide consistent and highly correlated measurements. The collocated operation of the HR-AMS and C-AMS during 11 days of SOAR$1(8 / 3-8 / 13 / 2005)$ provides an opportunity to make, to our knowledge, the first published intercomparison of two ToFAMSs. Measured HR-AMS and C-AMS total (i.e., the sum of all speciated NR-PM 1 measurements without EC) NR$\mathrm{PM}_{1}$ are shown in Fig. 3a along with $\mathrm{PM}_{2.5}$ TEOM ${ }_{\mathrm{FDMS}}$ measurements for reference. HR-AMS total measurements are also plotted against those of the C-AMS in Fig. 3b, while each speciated HR-AMS measurement is plotted against those of the C-AMS in Fig. 3c-g. Linear regressions were performed using a fixed-zero intercept (confirmed for both ToF-AMSs by sampling ambient air through a HEPA filter and measuring the resulting signal of particle-free air) and are shown for each scatter plot. Overall, ToF-AMS total NR-PM $M_{1}$ measurements track each other and TEOM $_{\mathrm{FDMS}}$ $\mathrm{PM}_{2.5}$ closely throughout this period. Linear regression of total NR-PM $\mathrm{PM}_{1}$ yields a slope of 1.06 with high correlation $\left(r^{2}=0.83\right)$. Similar results are obtained from the comparison of speciated measurements including OA (slope $=1.11$; $\left.r^{2}=0.81\right), \mathrm{NO}_{3}(0.98 ; 0.89)$, and $\mathrm{NH}_{4}(0.97 ; 0.79), \mathrm{SO}_{4}$ (1.06; 0.77), and $\mathrm{Cl}(1.13 ; 0.77)$. In all cases the differences are smaller than the combined estimated ToF-AMS uncertainty of $\pm 25 \%$. Thus both instruments provided consistent and highly correlated total and speciated mass concentrations throughout the SOAR-1 comparison period. Results obtained here are similar to or, in some cases, slightly better than those obtained from the comparisons of Salcedo et al. (2005) and Hings et al. (2007) despite the fact that these earlier studies compared $30 \mathrm{~min}$ average measurements, whereas five min averages were used in the current comparison. This could perhaps be due to either physical separation $(\sim 30 \mathrm{~m})$ and the use of separate inlets in the case of Salcedo et al. (2005) or the comparison of Q- and C-AMS measurements in the case of Hings et al. (2007).

\subsection{Comparison of NR-PM 1 vs. $\mathrm{PM}_{2.5}$ inorganics}

HR-AMS NR-PM $\mathrm{NO}_{3}$ and $\mathrm{SO}_{4}$ concentrations are compared with corresponding $\mathrm{PM}_{2.5}$ IC-nitrate and IC-sulfate measurements, respectively, in Fig. S12. Similar comparisons of $\mathrm{Cl}$ and $\mathrm{NH}_{4}$ are not possible because $\mathrm{PM}_{2.5}$ concentrations of these species were not measured. $\mathrm{NO}_{3}$ measurements from both instruments are very highly correlated (Fig. S12a), with linear regression providing similar standard and recovery slope values near unity. The consistency of these different measurements given the differerence in size cut between the instruments suggests that only a small amount of $\mathrm{NO}_{3}$ is contained in supermicron particles. Diurnal plots of NR-PM 1 and $\mathrm{PM}_{2.5} \mathrm{NO}_{3}$ are shown in Fig. S12b. The diurnal profiles from the two techniques exhibit similar broad, bi-modal maxima during the morning and early afternoon, and minima during the late afternoon and early evening.

HR-AMS NR-PM $\mathrm{SO}_{4}$ is plotted against $\mathrm{PM}_{2.5}$ ICsulfate measurements in Fig. S12c while diurnal profiles of each are provided in Fig. S12d. In contrast to the consistency and high correlation of NR-PM $\mathrm{PM}_{1}$ and $\mathrm{PM}_{2.5} \mathrm{NO}_{3}$ measurements, comparison of $\mathrm{SO}_{4}$ measurements exhibits substantially higher scatter as evidenced by a low degree of correlation $\left(r^{2}=0.42\right)$ between the measurements. Due to this scatter, linear regression results differ widely depending on whether a fixed-zero intercept is used. In the absence of a fixed-zero intercept, a slope of 0.51 is obtained. A recovery slope of 0.82 is obtained when an intercept of zero is used, which is likely more accurate considering the amount of scatter associated between the two measurements, the fact that $\mathrm{SO}_{4}$ concentrations rarely decreased below $2 \mu \mathrm{g} \mathrm{m}{ }^{-3}$ during SOAR-1, and the fact that the zero values of the HR-AMS was regularly checked by sampling particle-free air using a HEPA filter. Much of the scatter between NR-PM 1 and $\mathrm{PM}_{2.5} \mathrm{SO}_{4}$ may be due to the different particle size fractions sampled by each instrument. It is worth noting, however, that Grover et al. (2008) compared a limited number of IC-sulfate and PC-BOSS sulfate measurements and found a similar low degree of correlation (0.16-0.37). Diurnal profiles of NR$\mathrm{PM}_{1}$ and $\mathrm{PM}_{2.5} \mathrm{SO}_{4}$ (Fig. S12d) do not exhibit prominent 

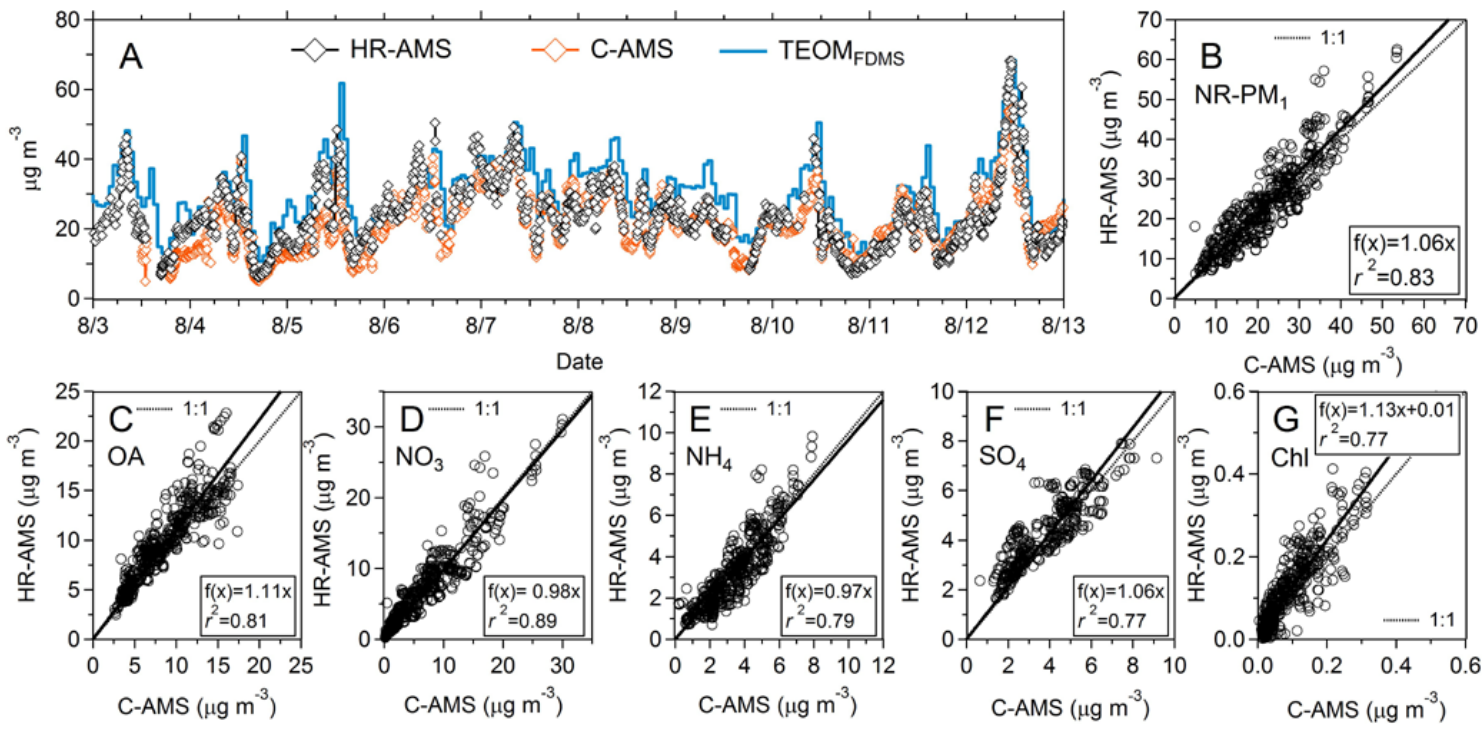

Fig. 3. Time series of NR-PM 1 concentrations measured by each ToF-AMS over the period 8/3-8/13-2005 are shown in panel A. TEOM $_{\text {FDMS }}$ concentrations are also provided for visual reference. Scatter plots of total and speciated NR-PM 1 are shown in panels B$\mathrm{G}$ with the results of linear regression and correlation $\left(r^{2}\right)$ values also provided in each plot.

features; instead, $\mathrm{SO}_{4}$ concentrations in each case are relatively constant throughout the day indicating the continuous presence of $\mathrm{SO}_{4}$ in the particles and its insensitivity to a range of ambient temperatures. Additionally, the presence of a supermicron $\mathrm{SO}_{4}$ fraction is suggested by $\mathrm{PM}_{2.5} \mathrm{SO}_{4}$ measurements being higher on average than corresponding NR-PM 1 measurements.

\subsection{Comparison of HR-AMS and Sunset OC}

HR-AMS OA and Sunset OC measurements cannot be compared directly. Instead, one measurement must be converted using an organic mass/organic carbon (OM/OC) ratio, which varies with OA composition (Turpin and Lim, 2001). Previous studies have converted AMS OA to OC using either a single estimated OM/OC ratio (Takegawa et al., 2005) or different OM/OC ratios for each component identified by factor analysis (Zhang et al., 2005a). Here, we apply a timedependent $\mathrm{OM} / \mathrm{OC}$ ratio determined from elemental analysis (see Sect. 4.7) to calculate and compare HR-AMS OC with three Sunset measurements as shown in Fig. 4. Two different measurements are available from Sunset 2 due to its separate measurement of semi-volatile OC (SVOC) (Grover et al., 2008). Herein, "Sunset2" corresponds to standard Sunset2 measurements (i.e., without SVOC correction) while "Sunset $2+$ SVOC" corresponds to Sunset2 OC measurements corrected for SVOC loss. Sunset1 measurements are shown as measured without any correction. Given the similar setup of both Sunset instruments during SOAR-1 (e.g., similar size cut and the use of charcoal-impregnated denuders upstream of both instruments to remove volatile organics) standard (i.e., without SVOC correction) measurements from both in- struments are similar in principle although differences such as individual sampling lines could still translate to measurement differences.

HR-AMS OC is plotted against Sunset1 measurements in Fig. 4a. Linear regression calculated using a fixed-intercept of zero results in a slope of 1.08 and moderate correlation $\left(r^{2}=0.53\right)$. Average OC concentrations during different periods throughout the day (i.e., 05:00-10:00, 10:0015:00, 15:00-20:00, and 20:00-05:00) are shown in Fig. 4b and are also quite similar. The results of this comparison are somewhat different than previous comparisons between PILS-OC and Sunset1 OC during SOAR-1 as reported by Peltier et al. (2007). In that case, linear regression provided a slope near unity with an intercept of $-1.69 \pm 0.22$, which was attributed to either a positive bias in the Sunset1 OC measurement or the inability of the PILS-OC to measure large insoluble particles (Peltier et al., 2007). The intercept is poorly determined in our regression due to nonzero OC background concentrations (i.e., no measurements $<2.5 \mu \mathrm{g} \mathrm{m}^{-3}$ ), so we cannot provide further insight into the possible reasons for that discrepancy. HR-AMS and Sunset 2 OC are compared in Fig. 4c. The slope obtained from regression with a fixed-zero intercept is near unity, although the data have a large amount of scatter as evidenced by the low degree of correlation $\left(r^{2}=0.36\right)$ between the measurements. As with Sunset1 measurements, average HR-AMS and Sunset2 OC concentrations are quite similar for the different periods shown in Fig. 4d. HR-AMS and Sunset2+SVOC are compared in Fig. 4e and average Sunset $2+$ SVOC concentrations throughout the day are shown in Fig. 4f. HR-AMS OC and Sunset2+SVOC measurements 

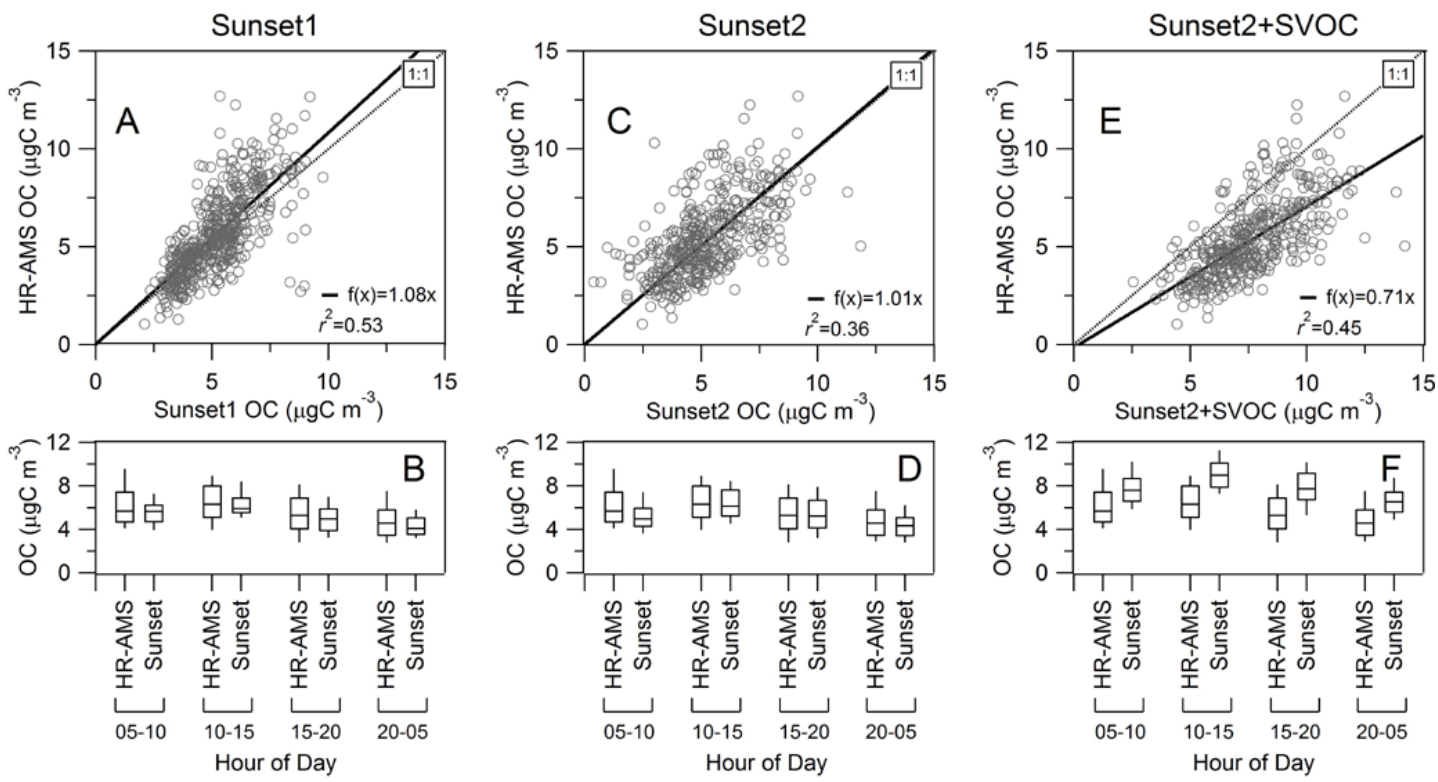

Fig. 4. Comparison of HR-AMS and Sunset OC concentrations. HR-AMS OC concentrations are plotted against Sunset1, Sunset2, and Sunset $2+$ SVOC along with results of linear regression and correlation values in panels A, C, and E, respectively. EA results (i.e., the diurnal profile of OM/OC) were used to convert HR-AMS OM to OC to allow these comparisons. Box and whisker plots located below each scatter plot compare the various percentiles of HR-AMS and Sunset OC throughout SOAR-1 for different time periods (05:00-10:00, 10:00-15:00, 15:00-20:00, and 20:00-05:00). Boxes correspond to 25th and 75th percentiles while whiskers correspond to 10th and 90th percentiles.

are more highly correlated relative to Sunset $2\left(r^{2}=0.45\right.$ vs. $0.36)$ and the slope obtained in this case $(m=0.71)$ is likely more precise given the lower amount of scatter between HRAMS OC and Sunset $2+$ SVOC. Sunset $2+$ SVOC concentrations, however, are consistently higher than both HR-AMS and Sunset1 measurements suggesting that the correction used here overestimates SVOC concentrations.

Comparison of EC, OC, and total carbon (TC) measured by both Sunset instruments (Fig. S13) reveals a substantially lower degree of correlation in each case than that observed between corresponding ToF-AMS NR-PM 1 OA measurements. EC (Fig. S5a) is the most highly correlated of the Sunset measurements with an $r^{2}=0.77$, however, the consistent slope of best fit lines (whether using a fixed intercept or not) of 0.63-0.64 shows a systematic difference between the two measurements. In contrast, a slope near unity is obtained from linear regression of standard Sunset OC (Fig. S13b) and TC (Fig. S13c) measurements using a fixed-zero intercept due to the low incidence of measurements $<3 \mu \mathrm{gC} \mathrm{m}{ }^{-3}$. However, regression results are likely influenced by the low degree of correlation between corresponding OC $\left(r^{2}=0.42\right)$ and TC $\left(r^{2}=0.59\right)$ measurement pairs. Results from linear regression of Sunset1 and Sunset2+SVOC OC (0.63, Fig. S13d) and TC (0.62, Fig. S13e) measurements are similar, substantially lower than unity and, like EC, indicate a systematic difference between the two Sunset instruments. In general, these results are unlike previous intercomparisons of Sunset EC (Park et al., 2006; Snyder and Schauer, 2007) and OC (Bae et al., 2004; Schauer et al., 2003) that found measurements from different instruments to be highly correlated.

Table S3 provides a summary of statistical comparisons between all OC measurements, including the difference between HR-AMS and Sunset OC measurements. Not only is the correlation between similar Sunset OC measurements equivalent to or lower than the correlation between HR-AMS and Sunset OC measurements, but the differences between either standard Sunset or Sunset2+SVOC concentrations are also as large or larger than those observed between HR-AMS and Sunset measurements. Diurnal profiles of HR-AMS and the various Sunset OC concentrations (Fig. S14a), and the absolute difference between the HR-AMS and each Sunset measurement (Fig. S14b), highlight similarities and differences among each OC measurement.

Overall, the average discrepancy between HR-AMS and Sunset 1 OC is $\sim 10 \%$ most of which could partially arise from errors in converting HR-AMS OM to OC values. Aiken et al. (2008) found an average error of $6 \%$ associated with reconstructing OM/OC values from atomic ratios of laboratory standards. The slightly higher discrepancy observed here may be due to higher error in the conversion of ambient OA compared to standards, or it may suggest an additional source of error beyond that involved in OM to OC conversion. For example, the largest difference between HR-AMS and Sunset OC occurs during overnight and early morning hours. These periods correspond to periods with 

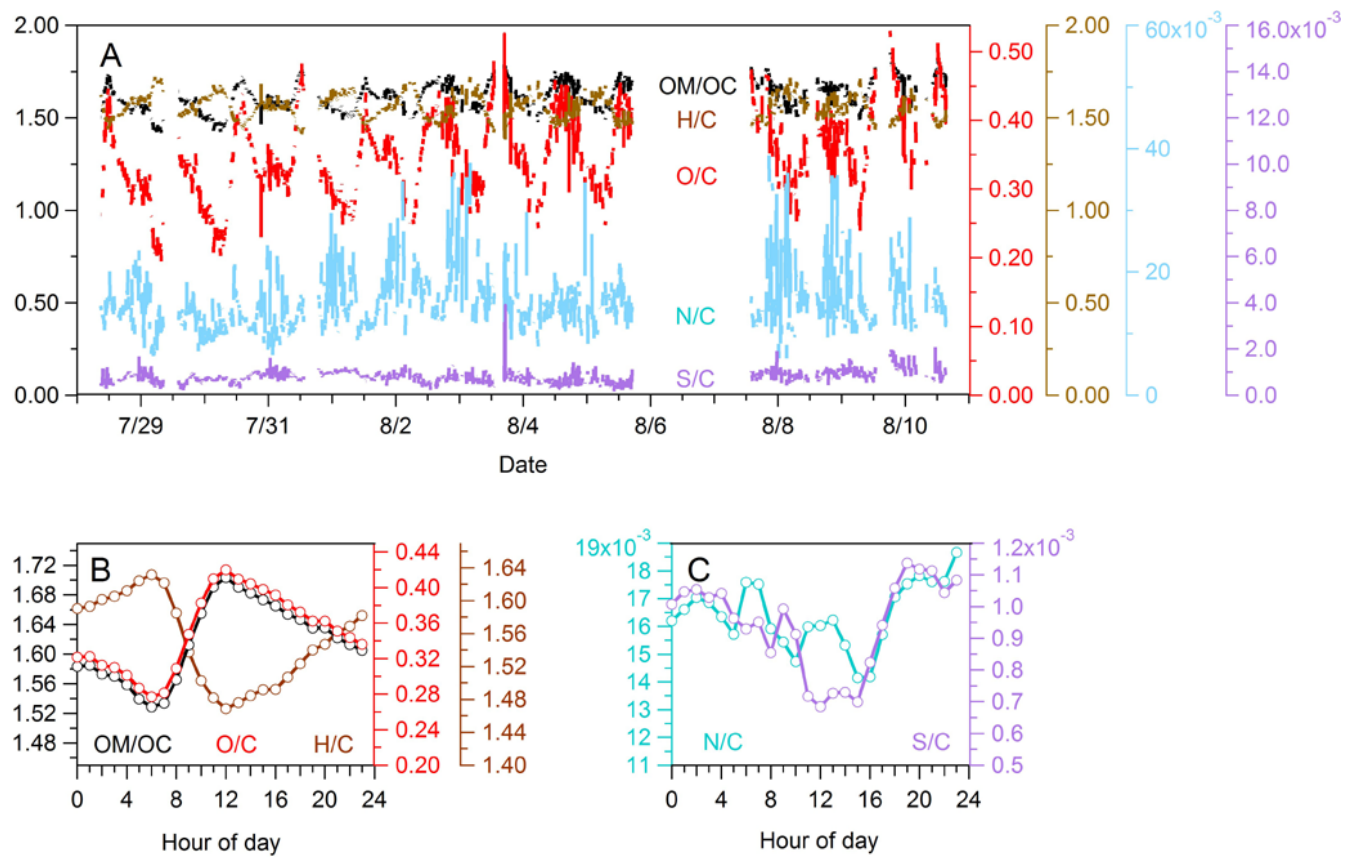

Fig. 5. Elemental analysis (EA) results for SOAR-1 OA. Time series and diurnal profiles of elemental ratios and organic mass to organic carbon $(\mathrm{OM} / \mathrm{OC})$ ratio are shown in panel a. Panel b shows diurnal profiles of OM/OC, O/C, and $\mathrm{H} / \mathrm{C}$ while panel $\mathrm{C}$ shows diurnal profiles of $\mathrm{N} / \mathrm{C}$ and $\mathrm{S} / \mathrm{C}$ ratios.

increased contributions of hydrocarbon-like organic aerosol (HOA) (Docherty et al., 2008), the volatility of which is on the higher end of OA components identified thus far (Huffman et al., 2009) thereby indicating potential evaporative losses on the part of the Sunset instruments. It should be noted, however, that even during these times, the difference between HR-AMS and the Sunset OC measurements are mostly within $\pm 15 \%$ and otherwise within $\pm 25 \%$ of the HR-AMS OC, which is within the uncertainties of both measurements. The difference between Sunset and HR-AMS measurements during overnight and morning hours could also be due, in part, to either a positive bias on the part of the HR-AMS due to potentially higher relative ionization efficiency (RIE) or collection efficiency (CE) for HOA (Jimenez et al., 2003; Zhang et al., 2005b; Aiken et al., 2009). If the difference were due entirely to such a bias, the observed difference between HR-AMS and Sunset OC suggest that the HR-AMS response could be $\sim 10-20 \%$ higher when sampling OA in which HOA and oxidized OA (OOA) contribute equally. This difference should be considered an upper limit, as other effects such as biases in HR-AMS EA, small differences in size cuts, variations in OC artifacts in the Sunset filter, or a small variation of the HR-AMS CE due to nitrate could also contribute to the observed time dependence. We recommend that similar comparisons are carried out in other studies to evaluate whether this variation is consistent across locations.

The composition of OA during the mid- to late afternoon throughout SOAR-1 is dominated by more highly oxidized aerosol types such as OOA which tends to have lower relative volatility (Huffman et al., 2009). Differences in HRAMS and Sunset OC are considerably smaller during those hours. Large measurement differences during times that are impacted by higher-volatility species, and conversely, low measurement differences during periods dominated by low-volatility species, further suggest a loss of SVOC from standard Sunset measurements leading to the observed differences between HR-AMS and Sunset OC. We would expect, therefore, minimal difference between HR-AMS and Sunset2+SVOC measurements during hours when the OA composition is dominated by SOA. Consistently higher Sunset $2+$ SVOC concentrations over the entire 24 -h period and particularly during overnight and early morning hours (ref. Fig. 4f), however, make this difficult to conclusively address.

\subsection{Elemental analysis (EA) of HR-AMS OA}

EA of HR-AMS OA mass spectra has been used to characterize ambient (Aiken et al., 2008) and smog chamber OA (Shilling et al., 2009; Chhabra et al., 2010). EA results for SOAR-1 OA are presented in Fig. 5. Most elemental ratios show little day-to-day variability, indicating a consistent OA composition during this period. Diurnal profiles provided in Fig. 5b-c show these trends more clearly. Diurnal profiles of $\mathrm{OM} / \mathrm{OC}$ and $\mathrm{O} / \mathrm{C}$ ratios are broadly anti-correlated with $\mathrm{H} / \mathrm{C}$, as expected from the low contributions of $\mathrm{N}$ and $\mathrm{S}$ to $\mathrm{OM}$ in this study (see below). OM/OC and O/C exhibit 
minima at 07:00 and maxima at 12:00. Following the midday maximum, average $\mathrm{OM} / \mathrm{OC}$ and $\mathrm{O} / \mathrm{C}$ values steadily decrease throughout the late afternoon, while average $\mathrm{H} / \mathrm{C}$ values increase from their minimum values. Several characteristics of the EA ratios are similar to those observed by Aiken et al. (2008) in Mexico City. For example, OM/OC and $\mathrm{O} / \mathrm{C}$ are similar since oxygen is the dominant heteroatom at both locations. Average (range) of $\mathrm{O} / \mathrm{C}$ and $\mathrm{OM} / \mathrm{OC}$ values for SOAR-1 are $0.35(0.19-0.53)$ and $1.62(1.42-1.85)$, respectively. These values are both slightly lower than $\mathrm{O} / \mathrm{C}$ (0.42) and OM/OC (1.72) obtained by Aiken et al. (2008). The lower latitude and intense photochemistry of Mexico City and the presence of BBOA $(\mathrm{O} / \mathrm{C}=0.31)$ in that study (Aiken et al., 2009) but not SOAR-1 may explain these differences. The average OM/OC value obtained from SOAR-1 $\left(1.6 \pm 0.2 \mu \mathrm{g} \mu \mathrm{gC}^{-1}\right)$ is similar to the recommended value of Turpin and Lim (2001) for urban areas. The relative changes of $\mathrm{O} / \mathrm{C}$ and $\mathrm{H} / \mathrm{C}$ also follow a slope of -1 as recently reported by Heald et al. (2010).

Results from Mexico City indicate that $\mathrm{O} / \mathrm{C}$ obtained from EA is correlated with the ratio of unit resolution $\mathrm{m} / z$, 44 to total OA signal $\left(f_{44}\right)$, which can be used to estimate $\mathrm{O} / \mathrm{C}$ from unit-resolution data in the field (Aiken et al., 2008). As shown in Fig. S15, $f_{44}$ and O/C ratios of SOAR-1 bulk $\mathrm{OA}$ are consistent with this observation and follow a similar general trend. Linear regression of SOAR-1 values is similar, although slightly lower $(\sim 8 \% \pm 9 \%)$, for the range of $\mathrm{O} / \mathrm{C}$ observed during this campaign. Although the intercept is substantially lower than observed in Mexico City, its value is poorly constrained during SOAR-1 as no measurements were observed at very low average $\mathrm{O} / \mathrm{C}$ due to the continuous dominance of SOA.

A variety of $\mathrm{N}$ - and S-containing compounds can contribute to the N/C and S/C ratios of ambient aerosol. For instance, characteristic mass spectral fragments have indicated the presence of amines in particles sampled in a number of locations (Murphy et al., 2007; Angelino et al., 2001). Additionally, $\mathrm{N}$-containing products formed from amines (Murphy et al., 2007) have been observed in SOA from chamber reactions. Organic nitrates $(\mathrm{ON})$ have been suggested to contribute to the $\mathrm{N}$ content of ambient OA based on smog chamber results (Matsunaga et al., 2009; Fry et al., 2009); however, ON may be destroyed in the atmosphere by hydrolysis reactions (Day et al., 2010). N from ON is not included in EA as carried out here, since most of the $\mathrm{N}$ therein is contained in $\mathrm{NO}_{\mathrm{x}}$ ions which are assigned to "nitrate" and not OA when using standard HR-AMS field data analysis procedures (Farmer et al., 2010). Similarly, S from species such as sulfonic acids will be captured by our analysis, but $\mathrm{S}$ arising from organosulfates (OS) is assigned to "sulfate" and is not separately quantified in standard HR-AMS field analysis (Farmer et al., 2010).

As shown in Fig. 5a, the contribution of $\mathrm{N}$ and $\mathrm{S}$ (excluding ON and OS) to OA mass in Riverside is far smaller than that of $\mathrm{O}$ with $\mathrm{N} / \mathrm{C}$ and $\mathrm{S} / \mathrm{C}$ ratios being between one and two orders of magnitude lower than $\mathrm{O} / \mathrm{C}$ ratios, respectively. $\mathrm{S} / \mathrm{C}$ was calculated using a calibration factor of 1.0 and obtained ratios should be considered a lower bound, since $\mathrm{S}$ is an electronegative element and similar to $\mathrm{O}$, it may have a somewhat reduced tendency to retain the charge during the ion fragmentation process (Aiken et al., 2007), although this effect should be far smaller than an order of magnitude. Average N/C and S/C ratios of 0.016 and 0.001 , respectively were obtained by EA. Diurnal profiles of N/C and S/C are shown in Fig. 5c. Both ratios show highest values during overnight hours when the site experienced low speed, variable winds.

Not including the contributions of $\mathrm{ON}$ and $\mathrm{OS}$ to $\mathrm{NO}_{\mathrm{x}}^{+}$and $\mathrm{SO}_{\mathrm{x}}^{+}$ions, respectively, can have a large impact on calculated elemental ratios, particularly in the case of S/C. To evaluate this impact we recalculate these ratios using the estimates of Farmer et al. (2010), i.e., assuming that ON contributes $\sim 10 \%$ of $\mathrm{NO}_{3}$ and $\mathrm{OS}$ contributes $\sim 12 \%$ of $\mathrm{SO}_{4}$ during SOAR-1. Using these values we estimate average N/C and $\mathrm{S} / \mathrm{C}$ ratios of 0.032 and 0.024 , respectively. Although both ratios are still an order-of-magnitude below O/C, N/C doubles and S/C increases by a factor of $\sim 24$ when including the estimated $\mathrm{ON}$ and $\mathrm{OS}$, respectively. Also $\mathrm{H} / \mathrm{C}$ increases only minimally $(<1 \%)$ and $\mathrm{O} / \mathrm{C}$ increases by $21 \%$ when ON and OS estimates are taken into account. Note that according to Farmer et al. (2010), the oxygen which is bonded to a carbon in ON and OS is detected by the HR-AMS as part of "organic" ( $C$-containing) fragments and is thus included in the $\mathrm{O} / \mathrm{C}$ of $\mathrm{OA}$ in the standard EA procedures, while only the $-\mathrm{NO}_{2}$ part of $-\mathrm{ONO}_{2}$ and the $-\mathrm{SO}_{3}$ part of $-\mathrm{OSO}_{3}$ are not included in standard EA since they are not bound to a carbon when detected. In summary, these estimates suggest that neglecting ON and OS in the standard EA method can result in a minor difference in $\mathrm{H} / \mathrm{C}$, substantial increases in $\mathrm{O} / \mathrm{C}$ and N/C, and an order-of-magnitude increase in S/C in urban areas. We recommend that these conclusions are evaluated at other locations, especially as direct ON and OS measurements become available.

\subsection{PMF analysis of $O A$ composition}

Separate PMF analyses of HR-AMS data excluding (AMSPMF) and including (TD-AMS-PMF) thermally denuded sampling periods were conducted to investigate both the composition of NR-PM 1 OA during SOAR-1 and the potential for the TD-AMS-PMF analysis to improve the resolution of OA components. PMF solutions containing one through twelve factors from each analysis were investigated in detail to determine that solution from either analysis that best determines the composition of SOAR-1 OA. Details regarding the comparison of AMS-PMF and TD-AMS-PMF solutions as well as criteria for choosing the best solution are provided in the Supporting Information. Here, we describe the composition of OA as resolved from the 7-component TD-AMS-PMF solution. 

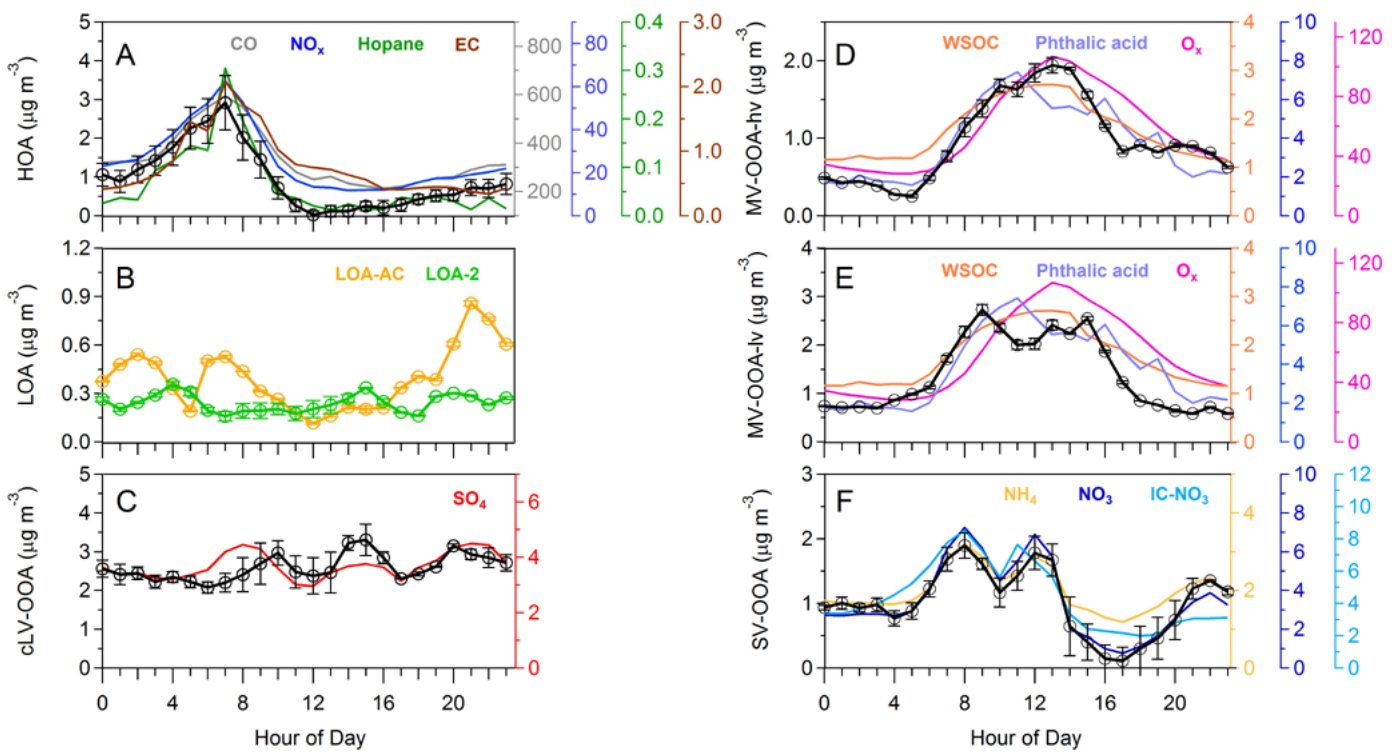

Fig. 6. Diurnal profiles of OA components identified in the 7-component TD-AMS-PMF solution. Error bars correspond to the standard error of the average hourly concentration calculated using the final, $p-1, p$, and $p+1$ solutions (standard errors for MV-OOA-hv and MVOOA-lv were calculated using final and $p+1$ solutions only). Concentrations of gas phase $\left(\mathrm{CO}, \mathrm{NO}_{\mathrm{x}}\right.$, and $\left.\mathrm{O}_{\mathrm{x}}\right)$ species are reported in ppbv. Concentrations of particulate carbon (EC and WSOC) and inorganic $\left(\mathrm{SO}_{4}, \mathrm{NH}_{4}, \mathrm{NO}_{3}\right.$, and IC-nitrate) species are reported in $\mu \mathrm{gC} \mathrm{m}^{-3}$ and $\mu \mathrm{g} \mathrm{m}^{-3}$, respectively, while particle associated hopane and phthalic acid concentrations are reported in arbitrary units.

\subsubsection{Hydrocarbon-like OA (HOA)}

Several factorization methods including custom principle component analysis (Zhang et al., 2005a), multi-component analysis (Zhang et al., 2007), and PMF (Ulbrich et al., 2009) of NR-PM ${ }_{1}$ OA from a wide variety of locations have consistently identified the presence of HOA. In each case, the spectra of this component are generally consistent and dominated by a reduced alkane fragment series that includes prominent fragments such as $m / z 57$ (mostly $\mathrm{C}_{4} \mathrm{H}_{9}^{+}$), which is a common fragment observed in spectra of reduced hydrocarbon standards (e.g., fuel and lubricating oil, Canagaratna et al., 2004) and ambient OA during periods heavily influenced by emissions from combustion sources (e.g., during morning rush hour). $m / z 57$ in ambient $\mathrm{OA}$ is strongly correlated with gas- and particle-phase tracers of primary emissions including $\mathrm{CO}, \mathrm{NO}_{\mathrm{x}}$, and EC (Zhang et al., 2005a). Due to the prominence of $\mathrm{m} / \mathrm{z} 57$ and other reduced alkane fragments in its spectrum and their strong correlation with primary tracer species, HOA is considered to be associated with primary emissions and to be analogous to POA.

On average, HOA contributes $13.8 \%$ of $\mathrm{NR}-\mathrm{PM}_{1}$ OA mass during the time period encompassed by the TD-AMSPMF analysis. Assuming a similar relative contribution over the entire duration of SOAR-1 yields an average HOA concentration of $\sim 1.20 \mathrm{\mu g} \mathrm{m}^{-3}$. Diurnal profiles of HOA and primary tracer species (e.g., $\mathrm{CO}, \mathrm{NO}_{\mathrm{x}}, \mathrm{EC}$, and particleassociated hopanes measured by TAG (Williams et al., 2010)) measurements over the duration of the TD-AMS-
PMF analysis are shown in Fig. 6a. Note that error bars in Fig. 6 correspond to the standard error of the mean calculated using the $(p-1)-, p$-, and $(p+1)$ solutions (where $p=$ number of factors in 7-component TD-AMS-PMF solution) and have been included to indicate the stability of component TS in the vicinity of our chosen solution. The corresponding TS of HOA and tracer species over a limited range of dates are also provided in Fig. S16a. Average HOA and primary tracer species concentrations track each other closely with a maximum in each occurring during the early morning commensurate with morning rush hour traffic. Following this maximum, HOA concentrations decrease quickly to a global minimum around noon. It is interesting to note that HOA and hopane concentrations at this time are nearly zero while $\mathrm{CO}, \mathrm{NO}_{\mathrm{x}}$, and $\mathrm{EC}$ concentrations are substantially higher. This could be due to the semi-volatile nature of HOA (Huffman et al., 2009) as discussed above resulting in partitioning of HOA to the gas phase during the hot mid-afternoon period. Gas-phase $\mathrm{CO}, \mathrm{NO}_{\mathrm{x}}$, and nonvolatile EC concentrations, on the other hand, are not susceptible to changes in ambient temperature and indicate that primary emissions continue, albeit with relatively lower contributions well into the early afternoon.

HOA emissions factors calculated from SOAR-1 are shown in Fig. S17 along with similar factors obtained from Pittsburgh (Zhang et al., 2005a), Mexico City (Aiken et al., 2009), and a tunnel environment (Ban-Weiss et al., 2008) where available. In general, the ratio of HOA to primary tracer species during SOAR-1 are of the same order as those 
determined from previous studies. HOA concentrations of Zhang et al. (2005a) and Aiken et al. (2009) were obtained through factorization of NR-PM 1 OA and emissions factors for those studies are analogous to those presented here. BanWeiss et al. (2008), on the other hand, measured total OA in the Caldecott Tunnel. Although OA in this case should be mostly primary in which case it would correlate with HOA obtained by factorization, emission ratios could be lower than the values observed in ackground urban air due to the contribution of sources with higher POA/CO and POA/ $\mathrm{NO}_{\mathrm{x}}$ ratios, such as food cooking, in the latter case. For example, the HOA:CO ratio during SOAR-1 $\left(4.62 \mu \mathrm{g} \mathrm{m}^{-3} / \mathrm{ppm}^{-3}\right)$ is between corresponding ratios obtained from sampling in Pittsburgh (4.30) and Mexico City (5.75) while the OA:CO ratio from the Caldecott Tunnel (3.10) is low by comparison. Similarly, the HOA: $\mathrm{NO}_{\mathrm{x}}$ ratio observed during SOAR$1\left(35.68 \mu \mathrm{g} \mathrm{m}^{-3} / \mathrm{ppm}^{-3}\right)$ is close to the value obtained from Pittsburgh (42.00) while the tunnel value (19.00) is again comparatively low. Finally, the HOA:EC ratios obtained from SOAR-1 (0.99 $\left.\mathrm{g} \mathrm{m} \mathrm{m}^{-3} / \mu \mathrm{gC} \mathrm{m}{ }^{-3}\right)$, Mexico City (1.25), and the Caldecott Tunnel (0.71) follow a similar trend. With respect to each tracer, SOAR-1 HOA concentrations are moderately correlated with values $\left(r^{2}\right)$ ranging from $0.52-$ 0.66. The correlation of HOA with the full complement of tracer species investigated are reported in Fig. S18. Overall, HOA is well correlated with the entire set of primary tracer species while correlations with secondary, mixed, and inorganic species are low.

The MS of HOA is shown in Fig. 7a along with results of EA and $f_{44}$ values. The MS of SOAR-1 HOA is characterized by a prominent alkane fragment series dominated by $\mathrm{C}_{\mathrm{x}} \mathrm{H}_{\mathrm{y}}^{+}$fragments including $\mathrm{C}_{4} \mathrm{H}_{9}^{+}$at $m / 2,57$ and is similar to spectra of combustion OA, fuel, and lubricating oil (ref. Fig. S19). EA results indicate a low contribution of oxygen $(\mathrm{O} / \mathrm{C}=0.019)$ and nitrogen $(\mathrm{N} / \mathrm{C}=0.003)$. Together with the highest $\mathrm{H} / \mathrm{C}$ ratio of any identified component (1.96), EA results indicate that HOA is composed nearly exclusively of carbon and hydrogen. The OM/OC value (1.20) of HOA obtained here is in good agreement with an equivalent estimate of molecular to carbon weight ratio $(\mathrm{MWt} / \mathrm{CWt}=1.20)$ for $n$ alkanes in the $\mathrm{C}_{24}-\mathrm{C}_{32}$ range (Turpin and Lim, 2001).

Along with HOA, two local OA (LOA) components resolved from the majority of TD-AMS-PMF solutions exhibit characteristics that do not indicate either their long distance transport or extensive atmospheric processing. Measured concentrations of the LOA components, for example, were highest and highly variable (varying by a factor of two within a period of 5-10 min ) during overnight periods which were characterized by low and variable wind speed and direction, respectively. These TS differ from those of other resolved OA components which exhibit sustained high concentrations over long periods with relatively small variability on shorter timescales. Autocorrelation profiles, showing the degree of correlation between each component TS and itself as one is shifted relative to the other in time clearly illustrate this dif- ference (ref. Fig. S20). Those components having high, relatively stable concentrations exhibit relatively flat autocorrelation profiles. For example, oxidized organic aerosol (OOA) component concentrations show little diurnal variability (see also text below) and the resulting autocorrelation profiles are relatively flat. In contrast, the autocorrelation profiles of each LOA component exhibit sharp declines on both sides of the origin with a full width, half height value of $\sim 6 \mathrm{~min}$. Based on an average overnight wind speed of $0.25 \mathrm{~m} \mathrm{~s}^{-1}$, this full width, half height value corresponds to an average plume width of $\sim 90 \mathrm{~m}$ suggesting sources relatively close to the sampling site. Characteristics of their MS also indicate that each LOA component is not highly oxidized, which again suggests these components represent relatively fresh emissions that have not been subject to atmospheric processing (Jimenez et al., 2009).

\subsubsection{Amine-containing LOA (LOA-AC)}

LOA-AC contributes $4.4 \%$ of OA mass in our final solution. The diurnal profile of LOA-AC (Fig. 6b) lacks a prominent diurnal profile due to its highly variable TS (ref. Fig. S16b). As a result, the LOA-AC TS has negligible correlations with any of the primary, secondary, and inorganic tracer species investigated (ref. Fig. S18) thereby providing few clues as its chemical identity and potential sources. On average, LOA$\mathrm{AC}$ concentrations are highest overnight and lowest during the daytime. The MS of LOA-AC, shown in Fig. 7b, is quite distinct from those of other resolved OA components. It is distinguished by prominent fragments characteristic of reduced amines, including $\mathrm{m} / \mathrm{z}, 86$ (mostly $\mathrm{C}_{5} \mathrm{H}_{12} \mathrm{~N}^{+}$). Additional fragments characteristic of reduced amines including $m / z$ 's $30\left(\mathrm{CH}_{4} \mathrm{~N}^{+}\right), 42\left(\mathrm{C}_{2} \mathrm{H}_{4} \mathrm{~N}^{+}\right), 56\left(\mathrm{C}_{3} \mathrm{H}_{6} \mathrm{~N}^{+}\right), 58$ $\left(\mathrm{C}_{3} \mathrm{H}_{8} \mathrm{~N}^{+}\right)$, and $84\left(\mathrm{C}_{5} \mathrm{H}_{10} \mathrm{~N}^{+}\right)$are also present. These ions are typically representative of both aliphatic acyclic $\left(\mathrm{C}_{n} \mathrm{H}_{2 n+2} \mathrm{~N}\right.$, e.g., at $\left.m / z, 30,58,86\right)$ and cyclic $\left(\mathrm{C}_{n} \mathrm{H}_{2 n} \mathrm{~N}\right.$, e.g., at $m / z 42,56,84$ ) alkylamines (McLafferty and Turecek, 1993). As expected based on the presence of these ions, the contribution of nitrogen to LOA-AC $(\mathrm{N} / \mathrm{C}=0.10)$ is substantially higher than in other identified components while a relatively low degree of oxidation is indicated by low $\mathrm{O} / \mathrm{C}$ $(0.143)$ and $f_{44}(0.03)$ values.

In addition to automobiles (Westerholm et al., 1993; Kean et al., 2000), a wide variety of sources including animal husbandry operations, marine sources, and biomass burning (Schade and Crutzen, 1995) contribute gas phase alkylamines to the atmosphere. During the summer months, Riverside is generally downwind of the Chino Air Basin which is home to several hundred dairy farms which emit substantial amounts of alkylamines. Assuming an alkylamine/ $\mathrm{NH}_{3}$ emission ratio similar to that determined by (Schade and Crutzen, 1995) and applying the ammonia emission rates of Lester and Woods (2004), these sources can potentially emit $\sim 0.13$ tons day ${ }^{-1}$ of amines. Lower mixing layer heights at night would increase alkylamine 

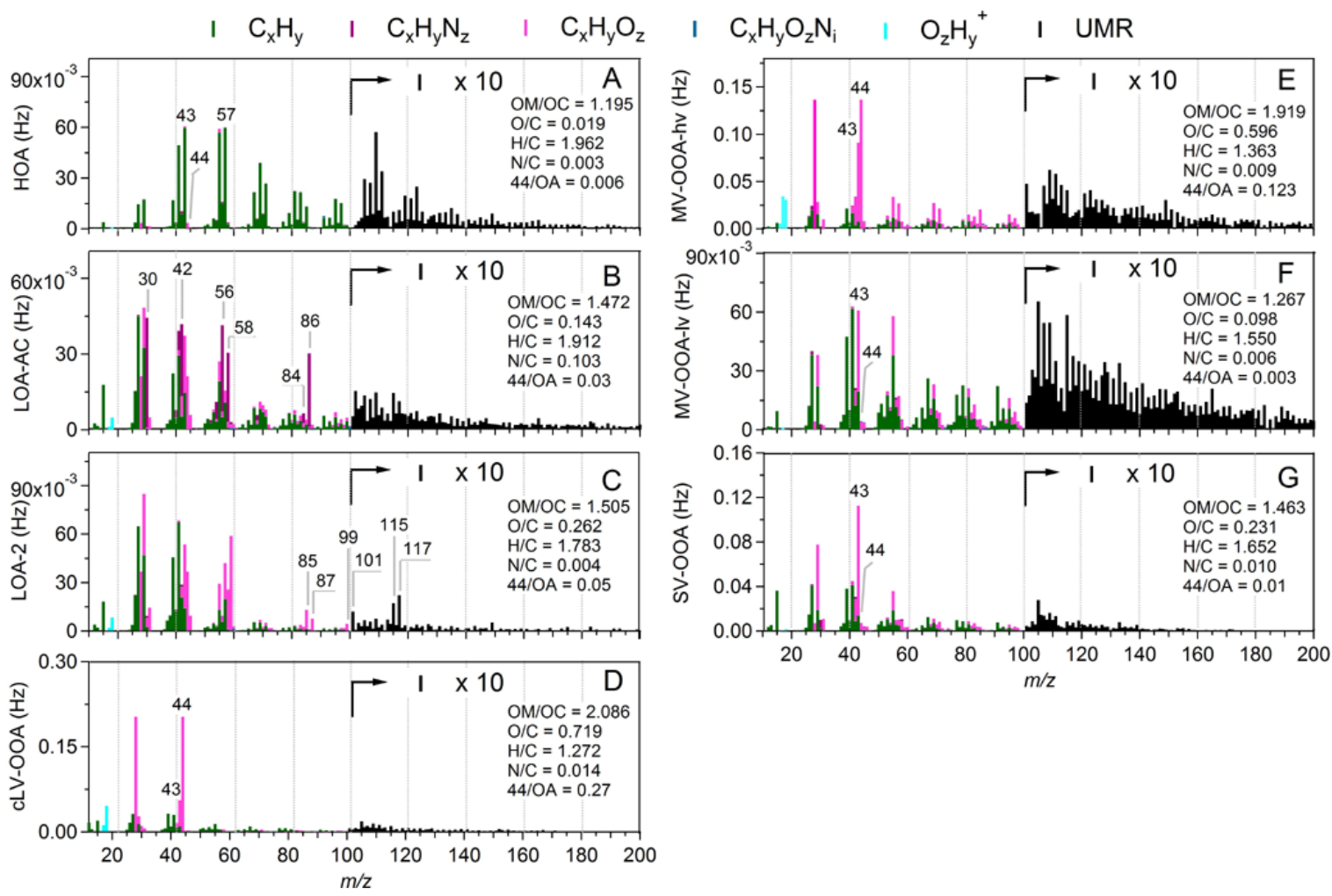

Fig. 7. Mass spectra of OA components identified from the 7-component TD-AMS-PMF solution. The intensity of unit-mass-resolution signals $>m / z 100$ have been adjusted to clearly show structure in this range. EA results and $f_{44}$ for each identified component have also been included.

concentrations consistent with maximum nighttime LOAAC concentrations. Several ambient studies have identified the presence of characteristic amine spectral fragments in aerosol mass spectra from a variety of locations (e.g., Ge et al. (2011) and references therein) including Riverside (Angelino et al., 2001; Pratt et al., 2009a). With particular regard to the HR-AMS, characteristic alkylamine ions have previously been observed in PMF-resolved components following analysis of OA from both Mexico City (Aiken et al., 2009) and New York (Sun et al., 2011). Despite these observations, the importance of alkylamines as a source of particulate nitrogen and the mechanism for the incorporation of alkylamines in aerosol remain speculative. Proposed mechanisms for the partitioning of otherwise high volatility alkylamines include the formation of alkylaminium salts through acid-base chemistry and the oxidation of gas-phase amines.

Alkylamines have been shown to form low-volatility alkylaminium salts which can partition to the particle phase. For example, Murphy et al. (2007) found alkylamines can react rapidly with nitric acid in environmental chamber reactions forming alkylaminium nitrate. Although subject to significant uncertainty, dissociation constants for at least some aminium nitrate salts are thought to be lower than that of ammonium nitrate suggesting that alkylamines can efficiently compete with ammonium as a sink for atmospherically avail- able nitric acid (Murphy et al., 2007). Similar heterogeneous reactions of alkylamines with acidic sulfate aerosol have also been found to irreversibly form alkylaminium sulfates (Wang et al., 2010).

Formation of alkylaminium salts can also occur through displacement of ammonium in both ammonium nitrate (Lloyd et al., 2009) and ammonium sulfate (Murphy et al., 2007). Lloyd et al. (2009) found that displacement of ammonium by trimethylamine is competitive under ambient conditions over the course of a few hours with trimethylamine concentrations as low as $1 \mathrm{ppb}$. Reactions to investigate displacement in the case of ammonium sulfate were performed at relatively high alkylamine concentrations and, as a result, the ability of this reaction to compete under atmospheric conditions is not as well established (Murphy et al., 2007). However, experimental results have also been supported by both modeling (Barsanti et al., 2009) and ambient studies that have found large contributions of aminium salts to aerosol in coastal (Facchini et al., 2008; Muller et al., 2009) and interior regions (Sorooshian et al., 2008) particularly those downwind of major bovine sources (Smith et al., 2010). Smith et al. (2010) investigated the volatility of alkylaminium salts formed by combining a series of alkylamines (methyl-, dimethyl-, and trimethylamine) with both acetic and propanoic acid and found that the volatility of the 
resulting alkylaminium salts were lower than that of ammonium sulfate. As a result, these salts could readily partition to the particle phase and contribute the amine fragments seen in ambient spectra.

If the amine fragments observed here were due to the presence of aminium salt, one would reasonably expect that the LOA-AC component, or at least the characteristic amine fragments, would display both a relatively low volatility and moderate to high correlation with inorganic anions. These characteristics were attributed to SOAR-1 amine fragments $(\mathrm{m} / \mathrm{z}$ 86) observed by Pratt et al. (2009a). During periods of SOAR-1 when the A-ATOFMS was interfaced with the $\mathrm{TD}$, alkylamine fragments were observed even at the highest TD temperatures $\left(230^{\circ} \mathrm{C}\right)$ while their relative intensity were found to be well correlated with A-ATOFMS $\mathrm{NO}_{3}$ and $\mathrm{SO}_{4}$ signals, particularly at elevated TD temperatures. Pratt et al. (2009a) accordingly attributed amine fragments to aminium salts.

However, the volatility of $\mathrm{m} / \mathrm{z} 86$ observed by Pratt et al. (2009a) differs from that of Huffman et al. (2009) and the current analysis where $m / z, 86$ and LOA-AC had among the highest volatilities of any fragment or identified OA component, respectively. Thermograms of both $\mathrm{C}_{5} \mathrm{H}_{12} \mathrm{~N}^{+}$and LOA-AC resolved from the 7-component TD-AMS-PMF solution and are shown in Fig. S21b. As this plot shows, the mass fraction of $\mathrm{C}_{5} \mathrm{H}_{12} \mathrm{~N}^{+}$decreases quickly with only $\sim 20 \%$ remaining at $50^{\circ} \mathrm{C}$. Additionally, $\mathrm{C}_{5} \mathrm{H}_{12} \mathrm{~N}^{+}$is poorly correlated with both $\mathrm{NO}_{3}$ and $\mathrm{SO}_{4}$ (not shown). The relatively high volatility of prominent alkylamine fragments and LOA-AC combined with the low correlation of the alkylamine ions with inorganic anions does not indicate the presence of low volatility alkylaminium salts as the main form of alkylamines in SOAR-1 OA.

A second proposed route for the incorporation of alkylamines into aerosols is through atmospheric oxidation. SOA formed following oxidation of gas phase alkylamines with the hydroxyl radical and ozone (Angelino et al., 2001; Murphy et al., 2007) as well as with the nitrate radical (Silva et al., 2008; Malloy et al., 2009) have been investigated in smog chamber reactions. LOA-AC concentrations have low correlation with hydroxyl and ozone concentrations (e.g., due to low daytime concentrations). As a result, it is unlikely that these oxidants play a prominent role in the formation of LOA-AC. Due to its prominence at night, the formation of LOA-AC could be due to nitrate radical reactions. Rate constants for these reactions have not been measured and their relevance, as a result, under ambient conditions is not well established. However, MS of SOA formed from these reactions displays a number of similar reduced amine fragments, including $m / z$ 86, as well as fragments representative of oxidized organics (Erupe et al., 2008).

In SOA formed from nitrate reaction of trimethylamine, the most abundant alkylamine in the atmosphere, prominent fragments in HR-AMS MS include $\mathrm{C}_{2} \mathrm{H}_{6} \mathrm{NO}_{2}^{+}$(nominal $\mathrm{m} / \mathrm{z}$ 76) in addition to characteristic reduced amine ions
$\mathrm{C}_{3} \mathrm{H}_{8} \mathrm{~N}^{+}$(nominal $m / z$ 58) and $\mathrm{C}_{5} \mathrm{H}_{12} \mathrm{~N}^{+}(\mathrm{m} / z$ 86) (Erupe et al., 2008). If LOA-AC were due to similar reactions, its MS should display fragments characteristic of oxidized amines. However, ions representative of oxidized amines do not appear to be particularly prominent in the LOA-AC MS. High resolution MS at nominal $\mathrm{m} / \mathrm{z} 58,76$, and 86 are shown in Fig. S22 both during periods of low (Fig. S22a-c) and high (Fig. S22d-f) LOA-AC concentrations. As expected, the signal for each ion is low when the concentration of LOA-AC is low. Reduced amine ions (e.g., $\mathrm{C}_{3} \mathrm{H}_{8} \mathrm{~N}^{+}$and $\mathrm{C}_{5} \mathrm{H}_{12} \mathrm{~N}^{+}$) increase substantially during periods of high LOA-AC concentrations while oxidized amine ions either decrease slightly or are unchanged. The dominance of reduced alkylamine ions and the apparent absence of oxidized amine ions again provide little support for the formation of LOA-AC through the oxidation of amines by the nitrate radical.

The precise chemical nature and source of LOA-AC remain unclear at present. The apparent inconsistencies of A-ATOFMS and HR-AMS alkylamine characterizations, including relative volatility and correlation of characteristic amine fragments with inorganic species, highlight the need for additional studies on the chemical nature of these species in ambient aerosol as well as the difference between various instruments used for its characterization.

\subsubsection{LOA-2}

LOA-2 contributes $2.6 \%$ of OA mass in the 7-component TD-AMS-PMF solution. As with LOA-AC, the TS of LOA2 (ref. Fig. S16b) is highly variable with highest concentrations overnight on average although a small number of large spikes were occasionally observed during the day. Due to this variability, LOA-2 again lacks a prominent diurnal profile (Fig. 6b), has generally weak correlation with any of the potential tracer species investigated (ref. Fig. S18), and, as a result, the comparison of the LOA-2 TS with those of tracer species again provides little insight as to either the identity or potential sources of LOA-2.

The MS of LOA-2 is shown in Fig. 7c along with results of EA analysis and $f_{44}$ ratio. The MS is characterized by a small number of prominent oxygen-containing ion pairs such as those at $m / z 85\left(89 \% \mathrm{C}_{5} \mathrm{H}_{9} \mathrm{O}^{+}\right)$and $m / z 87$ $\left(89 \% \mathrm{C}_{5} \mathrm{H}_{11} \mathrm{O}^{+}\right), m / z 99\left(93 \% \mathrm{C}_{6} \mathrm{H}_{11} \mathrm{O}^{+}\right)$and $\mathrm{m} / z .101$, and $\mathrm{m} / \mathrm{z}, 115$ and $\mathrm{m} / \mathrm{z} 117$. The contribution of oxygen-containing ions $<m / z, 100$ and the $\mathrm{O} / \mathrm{C}$ ratio show that this component is moderately oxidized although this is not equally represented in the $f_{44}$ value. Many prominent fragments observed in the LOA-2 spectrum can also be found in MS of the majority of other OA components. As a result, these fragments do not, by themselves, provide a sufficient information to conclusively identify this OA component. 


\subsubsection{Composite low-volatility OOA (cLV-OOA)}

cLV-OOA is the largest single OA component contributing $31.3 \%$ of OA mass on average in the 7-component TDAMS-PMF solution. Compared with the majority of identified OA components, cLV-OOA concentrations are relatively stable throughout the day as shown by its diurnal profile (ref. Fig. 6c). The diurnal profile of NR-PM $\mathrm{PMO}_{4}$ is also provided in this figure for reference due to its correlation with cLV-OOA. cLV-OOA also has weaker correlations (ref. Fig. S18) with known secondary (e.g., $\mathrm{O}_{3}, \mathrm{O}_{\mathrm{x}}$, and WSOC) and mixed source tracer species due to high daytime concentrations for cLV-OOA and those specific tracer species. Correlations with known primary tracer species, $\mathrm{NH}_{4}$, and $\mathrm{NO}_{3}$ are negligible.

The MS of cLV-OOA, corresponding EA results, and $f_{44}$ are provided in Fig. 7d. The spectrum is dominated by $\mathrm{m} / \mathrm{z} 44$ $\left(98 \% \mathrm{CO}_{2}^{+}\right.$), followed by substantially lower $\mathrm{m} / \mathrm{z}, 43$ (84\% $\mathrm{C}_{2} \mathrm{H}_{3} \mathrm{O}^{+}$) and very low contributions from fragments in the $\mathrm{m} / \mathrm{z}>44$ range. Large contributions at $\mathrm{m} / \mathrm{z} 44$ and the highly oxidized nature of this component are reflected in both high $f_{44}(0.20)$ and $\mathrm{O} / \mathrm{C}(0.72)$ ratios, which are slightly higher than corresponding values for comparable components reported from studies at different sites ( $\mathrm{Ng}$ et al., 2010). It is worthwhile to note, however, that the majority of previous PMF analyses identified fewer OA components than are identified here and, as a result, cLV-OOA is likely to have smaller contributions from unresolved OA components relative to previous analyses. Given the likelihood that any unresolved components would have a lower degree of oxidation any such contributions would reduce the apparent degree of oxidation for comparable components. For example, in the analysis of HR-AMS data obtained from Mexico City, Aiken et al. (2009) identified a single OOA factor having a moderate degree of oxidation $\left(\mathrm{O} / \mathrm{C}=0.60 ; f_{44}=0.14\right)$. If we assume that this bulk OOA component is a mixture of the two most commonly identified OOA components (i.e., LVOOA and SV-OOA) and synthesize a comparable component here by combining CLV-OOA and SV-OOA identified here, we find that the $f_{44}$ ratio $(0.15)$ of this synthetic component is similar to that of the single OOA component obtained by Aiken et al. (2009).

The MS of cLV-OOA is similar to comparable components identified in ambient OA from other geographical locations and those of highly oxidized organic analogs measured in the laboratory. Correlations of the cLV-OOA MS with library spectra are reported in Fig. S19b. With few exceptions, the cLV-OOA spectrum is highly correlated with mass spectra of fulvic acid (Alfarra, 2004), aged rural aerosol (Alfarra et al., 2004), and LV-OOA components of ambient OA from other locations (Lanz et al., 2007; Ulbrich et al., 2009). In contrast, the cLV-OOA spectrum has low correlations with primary OA laboratory standards or PMF-resolved HOA component spectra.
Based on the highly oxidized nature of cLV-OOA it would be reasonable to expect its high solubility in water. Surprisingly, cLV-OOA is only moderately correlated with WSOC on average throughout the day. This appears to be inconsistent with previous studies investigating the relationship between WSOC and bulk OOA (i.e., comparable to the non-HOA component from a two-component PMF solution) that found the two to be highly correlated (Kondo et al., 2007). WSOC in polluted regions is either directly emitted by biomass burning or is formed through secondary atmospheric processes (Weber et al., 2007). As the contribution from biomass burning events was minimal during SOAR-1 (Docherty et al., 2008), the vast majority of measured WSOC should be due to SOA. However, when daytime (i.e., 06:0018:00) and overnight (18:00-06:00) cLV-OOA concentrations are compared with corresponding WSOC concentrations (ref. Fig S23a), a large disparity is observed. Overnight cLV-OOA measurements are highly correlated with WSOC while daytime cLV-OOA measurements are considerably less well correlated. This suggests that cLV-OOA contributes the bulk of WSOC during overnight periods while one or more OA component(s) contribute the bulk of WSOC mass during the day. Interestingly, the effect is not observed between cLV-OOA and $\mathrm{SO}_{4}$ during similar periods (ref. Fig. S23b).

\subsubsection{Medium-volatility OOA (MV-OOA) and subcomponents}

To our knowledge, this is the first time MV-OOA or a comparable component has been resolved from PMF analyses of NR-PM 1 OA. As discussed in the Supporting Information, a single MV-OOA component was resolved in many of the solutions, but two MV-OOA components were further resolved in the TD-AMS-PMF analysis due to their different volatilities: a higher-volatility MV-OOA (MV-OOA-hv) and; a lower-volatility MV-OOA (MV-OOA-lv). Each of these components has a very similar TS, but are distinguished by both different volatilities (ref. Fig. 16e) and different MS. Despite the similarity of their TS, the observed differences between the volatilities and spectra of these two components suggest that each is a unique OA component.

MV-OOA-hv contributes $13.1 \%$ to total OA mass in the 7-component TD-AMS-PMF solution. The diurnal profile of this component is shown in Fig. 6d. High concentrations are observed throughout the day with lower concentrations overnight. Due to its prominence during the day, MV-OOA-hv is highly correlated (ref. Fig. S18) with the majority of secondary OA marker species including WSOC $\left(r^{\mathrm{TS}}=0.75\right), \mathrm{O}_{\mathrm{x}}\left(r^{\mathrm{TS}}=0.71\right)$, and particle-associated phthalic acid $\left(r^{\mathrm{TS}}=0.75\right)$. Correlations with each of the primary tracer species are negligible while only weak correlations are observed with inorganic tracer species. MV-OOAlv contributes $17.1 \%$ of OA mass and has a diurnal profile (ref. Fig. 6e) and TS (ref. Fig. S16e) very similar to those of MV-OOA-hv. As a result, MV-OOA-hv is highly correlated 
with the same set of measured tracer species (e.g., WSOC, Ox, and phthalic acid). Daytime and nighttime MV-OOA-hv, MV-OOA-lv, and the combined MV-OOA component (i.e., sum of MV-OOA-hv and MV-OOA-lv) concentrations are plotted versus corresponding WSOC, $\mathrm{O}_{\mathrm{x}}$, and phthalic acid measurements in Fig. S24 while results of linear regression are provided in Table S4. Concentrations of each component have been converted to carbon-based concentrations using component-dependent OM/OC ratios obtained from EA.

MV-OOA-hv and MV-OOA-lv contribute substantially to daytime WSOC concentrations and may likely contribute to the low correlation between cLV-OOA and WSOC during the day. Interestingly, daytime concentrations of MV-OOA (in $\mu \mathrm{gC} \mathrm{m}^{-3}$ ) are nearly equal those of WSOC resulting in a regression slope near unity. Although cLV-OOA values are low during the day, they are not zero. As a result, the sum of MV-OOA and cLV-OOA (comparable to bulk OOA) is substantially higher than WSOC during the day as shown in Fig. S25. The same is true during overnight periods as well with the sum of MV-OOA and CLV-OOA amounting to $\sim 150 \%$ of WSOC during both periods. This is likely due to the presence of water-insoluble oxidized organic carbon (OOC) (Kondo et al., 2007). Kondo et al. (2007) found that 6-26\% of summertime OOC in Tokyo was water-insoluble based on comparisons of PILS-WSOC and AMS measurements while others have estimated that this fraction could account for as much as $60 \%$ (Favez et al., 2007). Data presented in Fig. S25 suggest that up to $50 \%$ of OOC in Riverside could be water-insoluble which is toward the upper end of the range of values suggested by Kondo et al. (2007) and Favez et al. (2007).

The MS of MV-OOA-hv and MV-OOA-lv are shown in Fig. $7 \mathrm{e}$ and $7 \mathrm{f}$, respectively, along with EA results and the value of $f_{44}$ in each case. $\mathrm{m} / z 43$ (mostly $\mathrm{C}_{2} \mathrm{H}_{3} \mathrm{O}^{+}$) and $\mathrm{m} / \mathrm{z} 44$ (mostly $\mathrm{CO}_{2}^{+}$) contribute prominently in the MS of MV-OOA-hv along with smaller contributions from additional oxidized fragments in the range $44>m / z>100$. As expected based on the presence of numerous oxidized fragments, $\mathrm{O} / \mathrm{C}(0.60)$ and $f_{44}(0.12)$ ratios indicate that MVOOA-hv is moderately oxidized with negligible contributions from nitrogen. In contrast, $\mathrm{C}_{2} \mathrm{H}_{3} \mathrm{O}^{+}$and $\mathrm{CO}_{2}^{+}$have very small contributions in the MS of MV-OOA-lv which instead tends to be dominated by $\mathrm{C}_{\mathrm{x}} \mathrm{H}_{\mathrm{y}}^{+}$fragments indicating a more reduced nature which is confirmed by low $\mathrm{O} / \mathrm{C}(<0.10)$ and $f_{44}(<0.01)$ ratios along with the highest $\mathrm{H} / \mathrm{C}(1.55)$ ratio of any identified OOA component. Due to these spectral characteristics, we cannot rule out the possiblity that MVOOA-lv represents aged POA advected from the Los Angeles area. The contribution of high mass (i.e., $m / z>100$ ) ions in the MV-OOA-lv MS is the largest among all of the resolved OA components. Fragments in this mass range, however, lack clear structure as was present in either HOA or LOA-2 spectrum. Although the spectrum of MV-OOA-lv is similar to some POA and aged POA spectra, its identification as a single MV-OOA factor in many PMF solutions and its time series consistent with SOA tracers suggests that the unusual spectrum resembling POA may be due to imperfect separation of the spectra in the PMF solution process. Although it is also possible that MV-OOA-lv actually represents aged POA, we consider that less likely, and in the remainder of the paper we continue to discuss it as an OOA component.

In general, the degree to which the MV-OOA subcomponents are oxidized and their volatility appears to be contrary to the generally accepted framework that lower volatilities are observed for components with a higher degree of oxidation (e.g., Jimenez et al., 2009 and references therein). The relatively low degree of oxidation and low volatility of MVOOA-lv along with the large contribution from higher mass fragments suggest that this component could be indicative of oligomers. As discussed previously, common oligomeric fragment patterns observed by Denkenberger et al. (2007) were found to accompany characteristic indicators of aged OA including oxidized OC, sulfate, and nitrate suggesting that these species are strongly associated with secondary OA. Although Denkenberger et al. (2007) did not provide a TS or diurnal profile of these oligomeric species, it is reasonable to assume that these species were likely highest during the day similar to MV-OOA-lv concentrations.

The MV-OOA-lv spectrum, however, does not appear to display the characteristic oligomer fragmentation patterns, which often include high molecular weight fragments $(\mathrm{m} / \mathrm{z}>$ 300 ) with a repetitive pattern suggesting a highly functionalized carbon backbone (Kalberer et al., 2004; Tolocka et al., 2004). These characteristics were noted by both Reemtsma et al. (2006) and Denkenberger et al. (2007) during SOAR1. The absence of these characteristics from the HR-AMS spectrum could potentially be due to the ensemble nature of the HR-AMS. Denkenberger et al. (2007) noted that oligomeric fragments exhibited strong correlation with particle size during SOAR-1 with signal intensities being largest in small $(\sim 100-200 \mathrm{~nm})$ particles. Unlike the single particle A-ATOFMS, the HR-AMS samples particles in the $50 \mathrm{~nm}$ $<d_{p}<1000 \mathrm{~nm}$ range (Liu et al., 2007) simultaneously and, as a result, characteristic oligomeric fragments present in the smallest particles may have been diluted by more abundant species.

In the end, the observed correlation of the MV-OOA subcomponent TS with those of available tracer species are insufficient in and of themselves to determine either the source or production mechanism of these OA components. However, we would note that, relative to MV-OOA-lv, MVOOA-hv appears to have a stronger relationship to the extent of photochemical processing in the atmosphere as indicated by measured $\mathrm{O}_{\mathrm{x}}$ concentrations (Herndon et al., 2007) while daytime concentrations of MV-OOA-lv are much more strongly correlated with measurements of the SOA tracer phthalic acid (Schauer et al., 2002; Zheng et al., 2002; Fraser et al., 2003) which can result from photochemical oxidation of polycyclic aromatic hydrocarbons such as naphthalene (Jang and McDow, 1997). While this would seem to 
be consistent given the oxidation state of each component, measured $\mathrm{O} / \mathrm{C}$ ratios of SOA formed secondary to PAH oxidation are substantially higher than that obtained here for MV-OOA-lv (Chhabra et al., 2010).

\subsubsection{Semi-volatile OOA (SV-OOA)}

SV-OOA contributes $14.4 \%$ of OA mass on average in the 7-component TD-AMS-PMF solution. The diurnal profile of SV-OOA (ref. Fig. 6f) tracks those of inorganic ammonium and nitrate closely. SV-OOA mass concentrations are also plotted versus $\mathrm{NH}_{4}, \mathrm{NO}_{3}$, and IC-nitrate in Fig. S26. As shown in Fig. S26 and Fig. S18, SV-OOA is highly correlated with both $\mathrm{NO}_{3}, \mathrm{NH}_{4}$, as well as NR-PM $\mathrm{Cl}$, consistent with previous characterizations of this component $(\mathrm{Ng}$ et al., 2010). Correlations with the majority of other primary, secondary, and mixed source tracer species measured during SOAR-1 are relatively low with the exception of phthalimide. The strong correlation between the MV-OOA components with phthalic acid and between SV-OOA and phthalimide suggest the influence of different oxidation mechanisms for polycyclic aromatic hydrocarbons in the atmosphere.

The MS of SV-OOA is shown in Fig. 7g along with EA results and $f_{44}$ value. In contrast to cLV-OOA and MVOOA-hv, $m / z, 44\left(\mathrm{CO}_{2}^{+}\right)$contributes minimally to the SVOOA spectrum whereas $\mathrm{m} / \mathrm{z} 43\left(88 \% \mathrm{C}_{2} \mathrm{H}_{3} \mathrm{O}^{+}\right)$, which is contributed by non-acid oxygenates including saturated carbonyl groups (McLafferty and Turecek, 1993) in addition to others, has large contributions. $\mathrm{C}_{\mathrm{x}} \mathrm{H}_{\mathrm{y}} \mathrm{O}_{n}^{+}$and $\mathrm{C}_{\mathrm{x}} \mathrm{H}_{\mathrm{y}}^{+}$ions in the $m / z>44$ range have smaller contributions with little substantial contributions from fragments in the $m / z>100$ range. SV-OOA is not very highly oxidized as evidenced by low $\mathrm{O} / \mathrm{C}(0.23)$ and $f_{44}(0.01)$ ratios. We note that the $\mathrm{O} / \mathrm{C}$ ratio and $f_{44}$ reported here are slightly lower than corresponding values reported by $\mathrm{Ng}$ et al. (2010). This could again be due to the lower likelihood of other unresolved components contributing to SV-OOA in this case. Notwithstanding the difference in these absolute values, the spectral characteristics of SOAR-1 SV-OOA are highly similar to those of identified comparable components reported by $\mathrm{Ng}$ et al. (2010). Correlations between the UMR SV-OOA spectrum and those of various laboratory and ambient OA types are provided in Fig. S19 and shows that the SV-OOA spectrum resolved from SOAR-1 is generally similar to comparable components identified from PMF analysis of ambient OA in Pittsburgh (Ulbrich et al., 2009) and, in particular, Zurich (Lanz et al., 2007).

The volatility of SV-OOA is among the highest among all of the OOA components resolved during SOAR-1 (Huffman et al., 2009) and is similar to that of HOA (ref. Fig. S21f) with $>90 \%$ of SV-OOA mass removed at TD temperatures just over $100^{\circ} \mathrm{C}$. The relatively high volatility of SV-OOA is consistent with its low concentrations during the late afternoon when temperatures were highest. Due to its low oxidation and relatively high volatility, SV-OOA has been charac- terized as an intermediate in the transformation of directlyemitted OA to the highly oxidized LV-OOA due to OA aging (Jimenez et al., 2009). The volatility profile of $\mathrm{NO}^{+}$is also shown in Fig. S21f. Interestingly, the volatility profile of SVOOA and $\mathrm{NO}^{+}$are nearly identical indicating that not only is SV-OOA highly correlated with nitrate in temporal space but in volatility space as well. It is tempting to attribute this SV-OOA to organic nitrates based on the similar volatilities and the similarity between relative contribution of SV-OOA to $\mathrm{OA}(\sim 14 \%)$ and the estimated contribution of $\mathrm{ON}$ to $\mathrm{OA}$ $(9.5-19 \%)$ (Farmer et al., 2010). However, the low N/C ratio $(0.01)$ for SV-OOA suggest that this is not the case.

\subsubsection{Summary of OA composition}

OA during SOAR-1 was dominated by OOA components including the previously identified LV-OOA and SV-OOA components and two newly identified MV-OOA components. Smaller contributions arise from components which are not highly oxidized including the commonly identified HOA and the local OA components LOA-AC and LOA-2. As expected, SOAR-1 aerosol was dominated by carbon, oxygen, and hydrogen, with much smaller contributions from nitrogen and sulfur. The contribution of carbon, oxygen, hydrogen, nitrogen, and sulfur to OA during SOAR-1 and their distribution among the resolved OA components are shown in Fig. 8. Note that the relative contribution of each atom to OA is indicated at the top of each pie chart both as a percentage of total $\mathrm{OA}$ and average mass concentration.

Carbon contributes $71 \%$ of OA $\left(6.38 \mu \mathrm{g} \mathrm{m}^{-3}\right)$ and is distributed throughout each identified component nearly proportional to its relative contribution to OA mass. Hydrogen, on the other hand, contributes only $9 \%\left(0.81 \mu \mathrm{g} \mathrm{m}^{-3}\right)$ to overall OA mass and has a similar distribution among the identified components with HOA having a slightly larger fraction of hydrogen due to its highly reduced nature. Oxygen contributes $18 \%\left(1.62 \mu \mathrm{g} \mathrm{m}^{-3}\right)$ of overall OA mass. Unlike the distribution of carbon and hydrogen, that of oxygen is heavily weighted toward the OOA components, particularly cLVOOA and MV-OOA-hv, which collectively contribute nearly half of total oxygen. Nitrogen and sulfur contribute only $2 \%$ and $0.01 \%$ of OA mass, respectively, during SOAR-1. In the case of nitrogen, LOA-AC contributes just over a third of the ON with large contributions also from the OOA components, particularly cLV-OOA (26\%). cLV-OOA contributes just under half $(46 \%)$ of all the organic sulfur followed closely by SV-OOA (34\%). As discussed above, however, the fragmentation of OS and ON to nominally inorganic fragments can lead to EA underestimating the relative contributions of nitrogen and sulfur to OA mass, particularly in the case of sulfate.

Potential regions of origin of each of these components are explored in the wind rose plots shown in Fig. 9. Meteorological conditions during SOAR-1 including wind speed and direction were similar from day to day. Wind speed was 

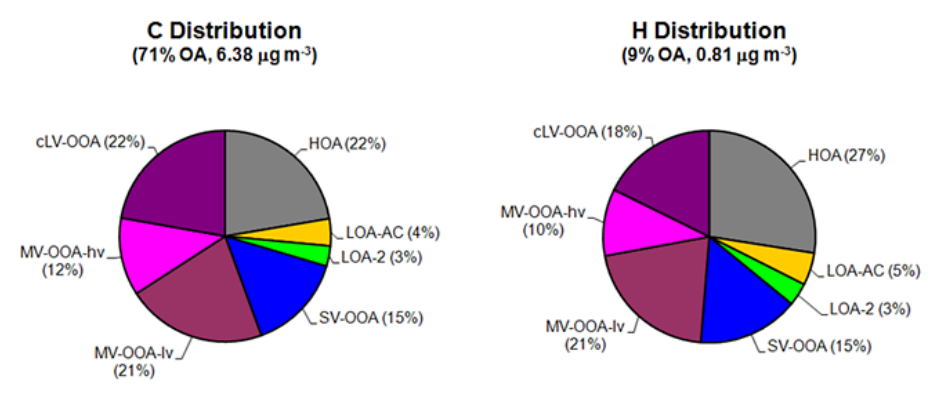

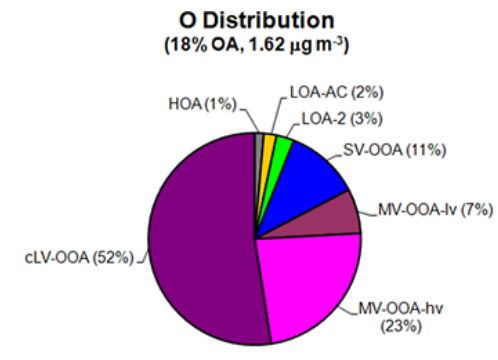

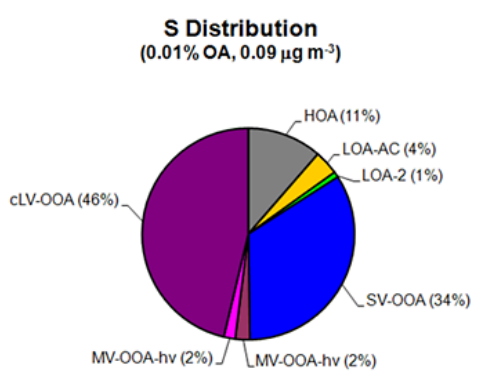

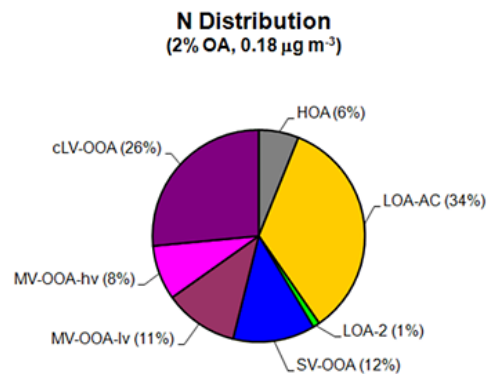

Fig. 8. Distribution of carbon, hydrogen, oxygen, sulfur, and nitrogen among OA components identified in the 7-component solution during SOAR-1.

low and direction variable during overnight periods but increased substantially out of the west during the day. In general, wind rose plots of HOA, LOA, and LOA-2 indicate that the sources of the components are more local as these components arrive from variable directions when wind speed was low and concentrations decrease during the day when wind speeds were highest. Specifically, HOA arrives to the sampling site from variable directions at night. As wind speeds increase, HOA generally arrives from the west indicating some HOA mass due to transport. However, HOA input is minimal as wind speed increases beyond $2 \mathrm{~m} \mathrm{~s}^{-1}$ indicating limited downwind transport. This pattern is consistent with the analysis of Williams et al. (2010) who identified two primary OA components that exhibit similar source patterns. LOA-AC and LOA-2 on the other hand arrive to the sampling site from a variety of directions exclusively when wind speed is low. Consistent with its occasionally large fluctuations during the day, LOA-2 appears to have a small inputs from downwind transport. The wind rose profile of LOA-AC provides little evidence to support that this component is subject to transport as inputs are essentially negligible as wind speeds increase $>1.5 \mathrm{~m} \mathrm{~s}^{-1}$.

Wind rose plots for the OOA components are substantially different and indicate that each of these components is transported into inland regions such as Riverside through long range transport. cLV-OOA (Fig. 9d) has some small inputs from variable directions when wind speed is low, but the vast majority of cLV-OOA mass arrives from the west when wind speeds increase beyond $1.5 \mathrm{~m} \mathrm{~s}^{-1}$. This same pattern is observed in wind rose plots of MV-OOA-hv, MV-OOA-lv, and SV-OOA as well. In the case of SV-OOA, some small inputs can be seen from variable directions at low wind speed indicating either a relatively more local source or a widespread presence of SV-OOA throughout the SoCAB.

\subsection{Average NR-PM + EC composition}

Time series and diurnal profiles for the relative contribution of major NR-PM 1 components and Sunset1 EC are shown in Figs. 10 and 11, respectively, while average concentrations for each component are also reported in Table S4. Overall, $\mathrm{OA}$ is the largest single component contributing nearly half of HR-AMS + EC mass on average. The majority ( $89 \%)$ of $\mathrm{EC}$ in the SoCAB during SOAR-1 should be due to diesel vehicles based on the reported emissions factors of Ban-Weiss et al. (2008) and CARB fuel usage data (California Air Resources Board, 2009). EC accounts for a few percent of the mass, with maximum concentrations in the early morning during rush hour as a result of both increased emissions and shallow mixing layer heights. EC concentrations decline after $\sim 08: 00$ mostly due to increased dilution due to breakdown of the nocturnal boundary layer at this time causing rapid downward mixing of cleaner air from aloft (Snyder et al., 2008). While absolute OA concentrations in the morning $\left(11.9 \mu \mathrm{g} \mathrm{m}^{-3}\right)$ and mid afternoon $\left(11.4 \mu \mathrm{g} \mathrm{m}^{-3}\right)$ are similar, the relative contribution of OA to HR-AMS + EC increases from $\sim 43 \%$ during morning rush hour to $\sim 55 \%$ during the late afternoon, mostly due to the lower contribution of $\mathrm{NH}_{4} \mathrm{NO}_{3}$ during the afternoon. The disparity between the diurnal cycles of EC and $\mathrm{OA}$ and $\mathrm{PM}_{f}$ is similar to that observed in Mexico City (Paredes-Miranda et al., 2009), and it is thought to be due mostly to dilution in the growing 


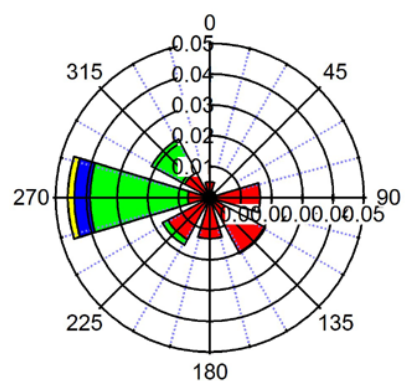

A. HOA

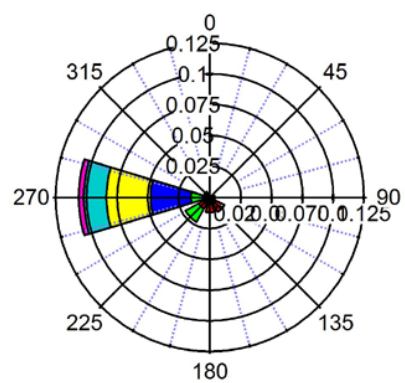

D. $c L V-O O A$

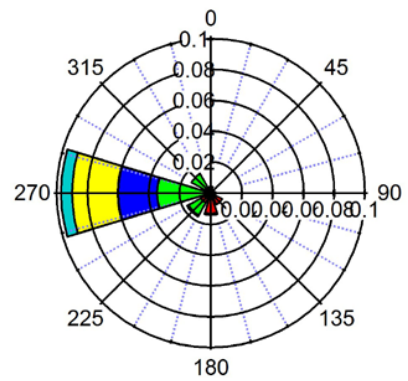

G. SV-OOA

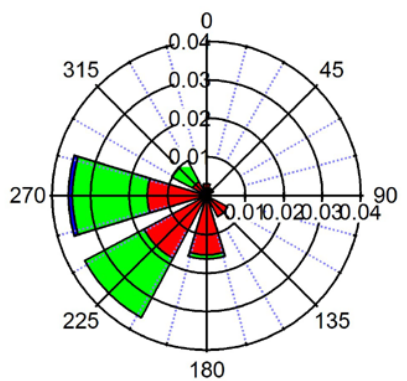

B. LOA-AC

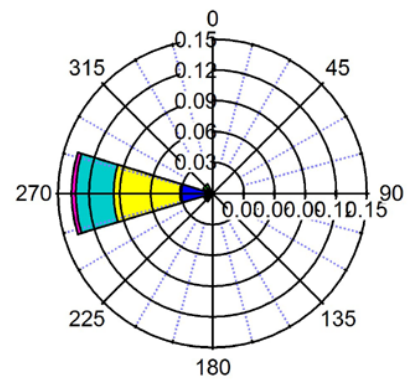

E. MV-OOA-hv

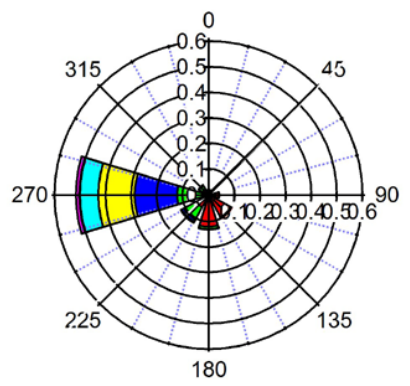

H. wind, generally

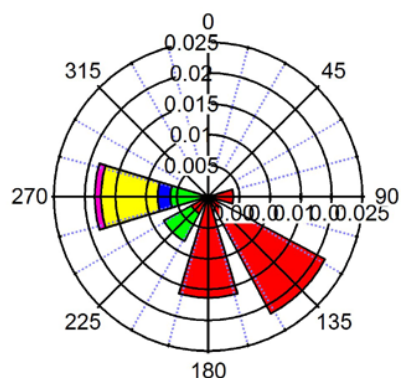

C. LOA-2

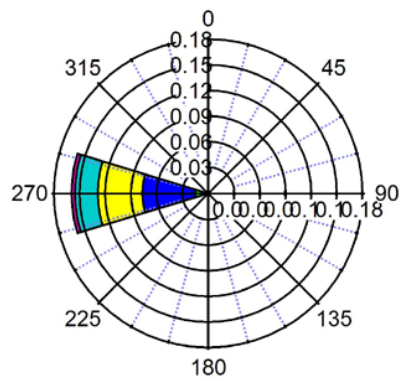

F. MV-OOA-IV

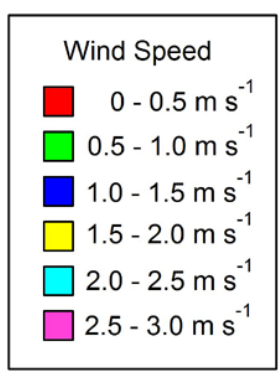

Fig. 9. Wind rose plots for OA components identified from the 7-factor TD-AMS-PMF solution.

boundary layer and SOA formation (Paredes-Miranda et al., 2009; Docherty et al., 2008).

Inorganics contribute the remainder of HR-AMS + EC mass with $\mathrm{Cl}$ contributing the smallest amount of mass $(0.44 \%)$ on average. Semi-volatile ammonium chloride is thought to be responsible for at least some of the HR-AMS chloride, since sodium chloride is thought to be poorly detected (Salcedo et al., 2006). The presence of semi-volatile chloride species is consistent with the diurnal profile during SOAR-1 which shows highest and lowest concentrations during overnight and morning periods and mid afternoon, respectively. While $\mathrm{SO}_{4}$ and $\mathrm{NO}_{3}$ contribute nearly equally to HR-AMS + EC throughout SOAR-1, the diurnal profiles of both their mass concentrations and relative contributions (ref. Fig. 11) display distinctly different behaviors consistent with these ions having different volatilities and sources. Average $\mathrm{SO}_{4}$ concentrations show little diurnal variation, which is consistent with its non-volatile nature and the ubiquity of particulate $\mathrm{SO}_{4}$ in inland regions of the SoCAB. This sug- gests that a larger fraction of $\mathrm{SO}_{4}$ originates from non-local production through regional secondary processes (Zhang et al., 2005b; DeCarlo et al., 2010).

Unlike $\mathrm{SO}_{4}, \mathrm{NO}_{3}$ concentrations are significantly elevated in inland $\mathrm{SoCAB}$ regions relative to locations closer to the coast (Neuman et al., 2003). The bulk of particulate $\mathrm{NO}_{3}$ in the vicinity of Riverside is due to secondary formation of $\mathrm{NH}_{4} \mathrm{NO}_{3}$. As mentioned above, $\mathrm{NH}_{4} \mathrm{NO}_{3}$ was determined to be among the most volatile aerosol components during SOAR-1. Due in large part to its relatively high volatility, $\mathrm{NO}_{3}$ has a strong diurnal profile (ref. Fig. 11a) that is very different from that of $\mathrm{SO}_{4}$ and is highlighted by its exhibiting two distinct maxima. The first occurs in the morning concurrent with rush hour while the second occurs in the early afternoon. This temporal behavior has been previously noted in the Riverside area (Stolzenburg and Hering, 2000; Fine et al., 2003). For example, Stolzenburg and Hering (2000) observed a morning maximum following an increase in gasphase nitric oxide during the morning rush hour but before an 


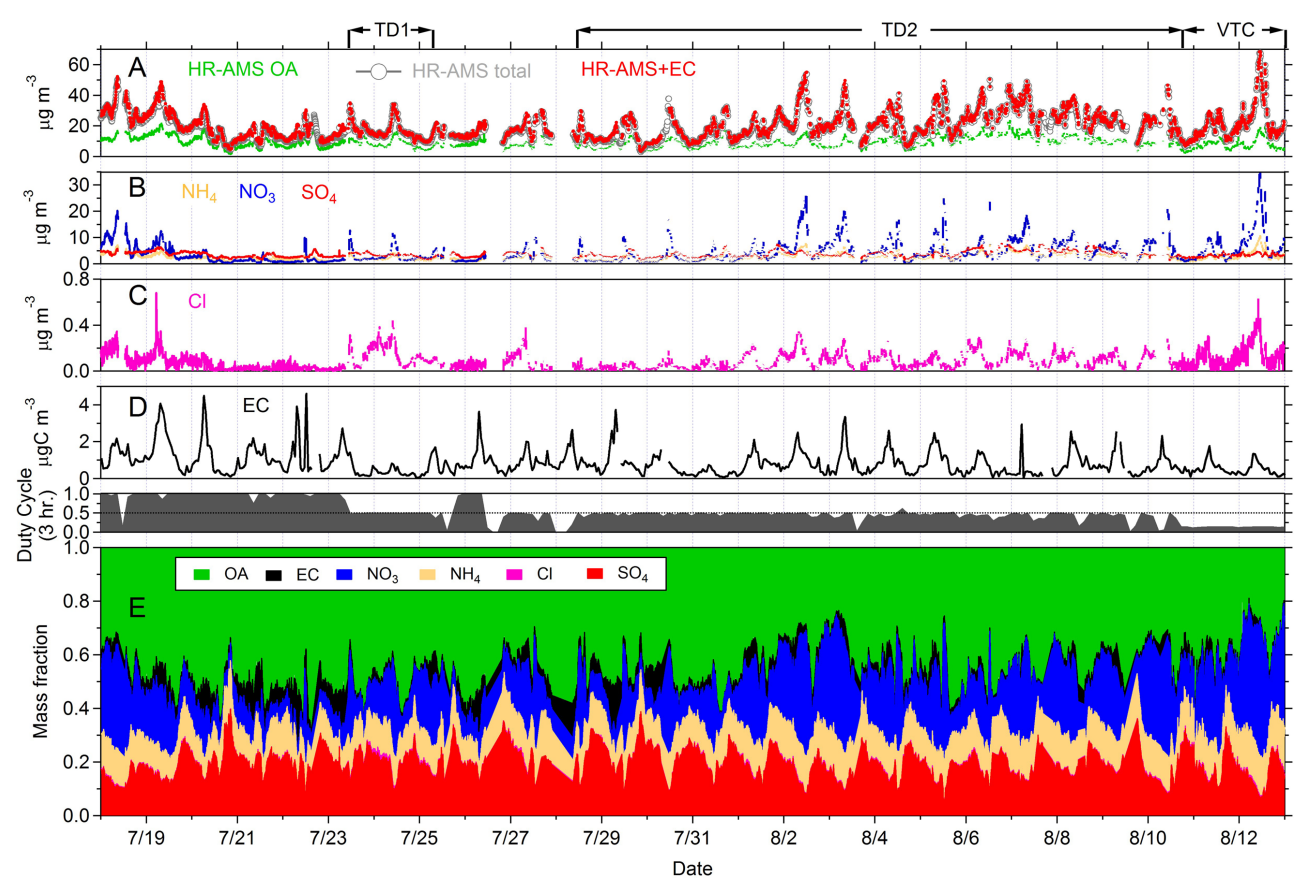

Fig. 10. Time series (Fig. 10a-d) and relative contributions of major NR-PM 1 species (Fig. 10e) to HR-AMS + EC during SOAR-1. The ambient sampling duty cycle of the HR-AMS (i.e., the fraction of time sampling under standard conditions) averaged over a period of 3 hrs. is shown in Fig. 10d. the use of thermodenuder (e.g., TD1, TD2) and vaporizer temperature cycling (VTC) protocols result in duty cycles of $50 \%$ and $\sim 13 \%$, respectively.

increase in ozone concentrations while the afternoon maximum coincided with the increase in ozone with the maximum occurring near the time of maximum daily temperature. Diurnal profiles of NR-PM $\mathrm{NO}_{3}$, nitric oxide, ozone, and ambient temperature during SOAR-1 are presented in Fig. S27. Similar to the observations of Stolzenburg and Hering (2000), average morning maximum $\mathrm{NO}_{3}$ concentrations during SOAR-1 occur at or just after maximum nitric oxide concentrations while the second maximum occurred just prior to maximum daily temperature and ozone measurements. Consistent with the semi-volatile nature of $\mathrm{NH}_{4} \mathrm{NO}_{3}$, minimum $\mathrm{NO}_{3}$ concentrations were observed during the late afternoon concurrent with maximum average daily temperatures and minimum relative humidities.

Diurnal profiles of NR-PM $\mathrm{P}_{1}$ OA components from the 7component TD-AMS-PMF solution and the relative contribution of each during SOAR-1 are also shown in Fig. 11. Diurnal profiles of absolute OA component concentrations along with the PMF residual is shown in Fig. 11e and $\mathrm{f}$ while the relative contribution of each is shown in Fig. 11f. Relative contributions from the identified LOA components are fairly minor and do not exhibit a pronounced diurnal pattern but have relatively low contributions throughout the day. HOA exhibits a prominent maximum during the early morning hours contributing $\sim 30 \%$ of NR-PM 1 OA mass at this time decreasing quickly to a noontime minimum of $\sim 3 \%$. Throughout the day, however, NR-PM 1 OA compo- sition is overwhelmingly dominated by contributions from OOA components with contributions from individual components varying over the course of the day. cLV-OOA has the largest overall contribution to OA throughout the day while that of the remaining OOA components range from $\sim 13$ $17 \%$ of total OA. The contribution of total OOA (i.e., sum of the individual OOA contributions) is $\sim 50 \%$ in the early morning and increases to over $90 \%$ just after noon. Interestingly, the relative contribution of SV-OOA decreases dramatically just in the late afternoon likely due to its shifting to the gas phase during the warmest time of the day as its high volatility would suggest.

\subsection{Anion/cation balance in NR-PM 1}

Ammonia $\left(\mathrm{NH}_{3}\right)$ is the principal anion in ambient aerosols, particularly in the SoCAB due to high emissions from a variety of sources. $\mathrm{NH}_{3}$ emissions in the basin originate from livestock and soils as well as from mobile, industrial, and domestic sources with livestock contributing approximately one-third of total emissions (Chitjian and Koizumi, 2000). The Chino/Ontario area is directly upwind of Riverside under prevailing wind conditions and is one of the most dense dairy cow populations in the United States having approximately 300 dairies with over 250,000 cows. Although a number of dairy operations have been relocated out of the basin in recent years, 314 operations remained as of 2002 with the 

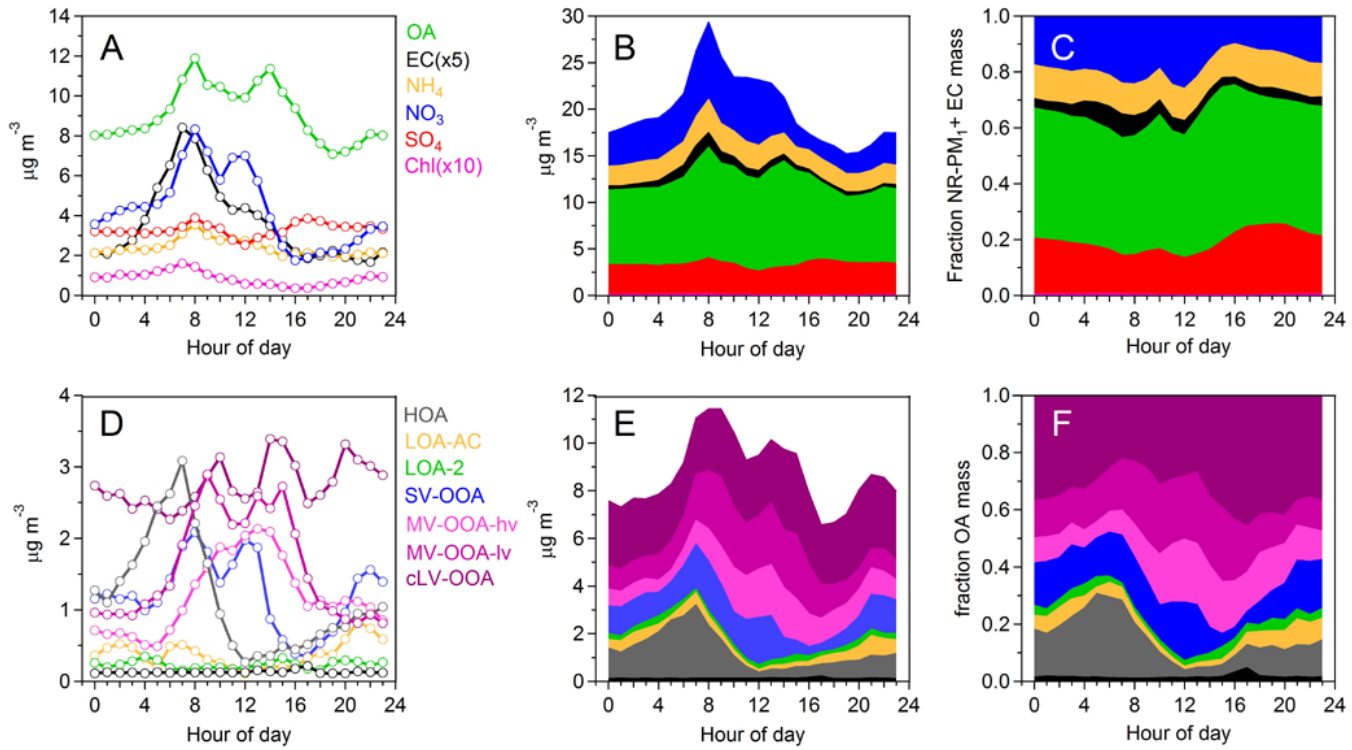

Fig. 11. Hourly average concentrations of major NR-PM 1 and EC (a-c), and OA components (d-f) identified from the 7-component solution during SOAR-1. Note that the absolute concentration of chloride has been multiplied by a factor of 10 in (a) for clarity. Diurnal profiles in (b) and e are stacked to show the overall composition of NR-PM 1 and OA, respectively, while profiles in (c) and (f) show the relative contribution of major NR-PM 1 and EC to HR-AMS and identified PMF components to OA, respectively. The black trace at the bottom of panels (d)-(f) is the PMF residual.

vast majority $(87 \%)$ being located in the Chino valley directly upwind of Riverside (Lester and Woods, 2004). Due to high $\mathrm{NH}_{3}$ emissions upwind and high concentrations in the area of Riverside, NR-PM 1 anionic species $\left(\mathrm{NO}_{3}, \mathrm{SO}_{4}\right.$, and chloride) and bulk aerosols should be largely neutralized. The charge balance of NR-PM 1 anions and cations measured during SOAR-1 is quantitatively evaluated in Fig. 12 where measured $\mathrm{NH}_{4}$ concentrations are compared against concentrations required for full neutralization of measured NR-PM cations, ignoring ON and OS (Zhang et al., 2005b) and using inorganic species concentrations determined from highresolution HR-AMS data. This comparison also assumes that mineral and sea salt cations (e.g. $\mathrm{Ca}^{2+}, \mathrm{Na}^{+}$) are low although some interference from dust is possible for SOAR-1. Overall, measured and predicted $\mathrm{NH}_{4}$ are highly correlated with $r^{2}=0.99$. Linear regression results in a slope of 0.86 while the ratio of average values is 0.91 indicating that essentially all of the $\mathrm{NO}_{3}$ and $\mathrm{SO}_{4}$ are present as ionic species and are neutralized by $\mathrm{NH}_{4}$ during SOAR-1 within the experimental uncertainty of this determination.

The predicted $\mathrm{NH}_{4}$ concentrations, however, are slightly larger than measured. The inset in Fig. 12 shows the range of concentrations $\left(<3 \mu \mathrm{g} \mathrm{m}^{-3}\right)$ where $\left(\mathrm{NH}_{4}\right)_{2} \mathrm{SO}_{4}$ represents nearly the entire contribution of NR-PM $\mathrm{NH}_{4}$. Linear regression of measured and predicted $\mathrm{NH}_{4}$ concentrations within this range results in a slightly larger slope (0.92) and smaller intercept (0.01) again suggesting that NR-PM $\mathrm{SO}_{4}$ was nearly completely neutralized. Additionally, this suggests that the deviation between measured and predicted $\mathrm{NH}_{4}$ is driven by slightly larger differences at higher $\mathrm{NH}_{4}$ concentrations (i.e., $>3 \mu \mathrm{g} \mathrm{m}^{-3}$ ) where the majority of $\mathrm{NH}_{4}$ is associated with $\mathrm{NO}_{3}$.

The presence of ON and OS as well as the unaccounted presence of other particulate cations may contribute to the observed difference between measured and predicted $\mathrm{NH}_{4}$. Fig. 12b investigates the impact of particulate OS, ON, and amines on the $\mathrm{NH}_{4}$ balance. To explore their impact on HRAMS ion balance, ON and OS have been assumed to contribute on the order of $10 \%$ and $12 \%$ of measured $\mathrm{NO}_{3}$ and $\mathrm{SO}_{4}$ mass and that $\mathrm{OS}$ is partially (here we assume $50 \%$ ) neutralized by $\mathrm{NH}_{4}$ (Farmer et al., 2010). The influence of amines on the HR-AMS ion balance is unknown. Amines may contribute to fragments nominally assigned to $\mathrm{NH}_{4}$ (e.g., $m / z, 16,17$, and 18) in which case they would artificially increase both measured "ammonium" and apparent particle basicity. As discussed above, amines can compete with $\mathrm{NH}_{4}$ for particulate anions such as inorganic $\mathrm{SO}_{4}$ and $\mathrm{NO}_{3}$ forming aminium salts (Murphy et al., 2007; Lloyd et al., 2009), thereby increasing nominal anion concentrations and apparent acidity. However, if alkylaminium salts produced $\mathrm{NH}_{\mathrm{x}}^{+}$ions with similar molar efficiency as $\mathrm{NH}_{4}$, the ammonium balance calculation would only be minimally affected by their presence, as they would contribute equal amounts of nominal anions and cations to the balance. In order to estimate an upper bound as to the impact of amines on the ion balance, we assume here that the detected amines do not contribute appreciable signal to fragments nominally assigned to $\mathrm{NH}_{4}$. The LOA-AC component identified in the 7- 

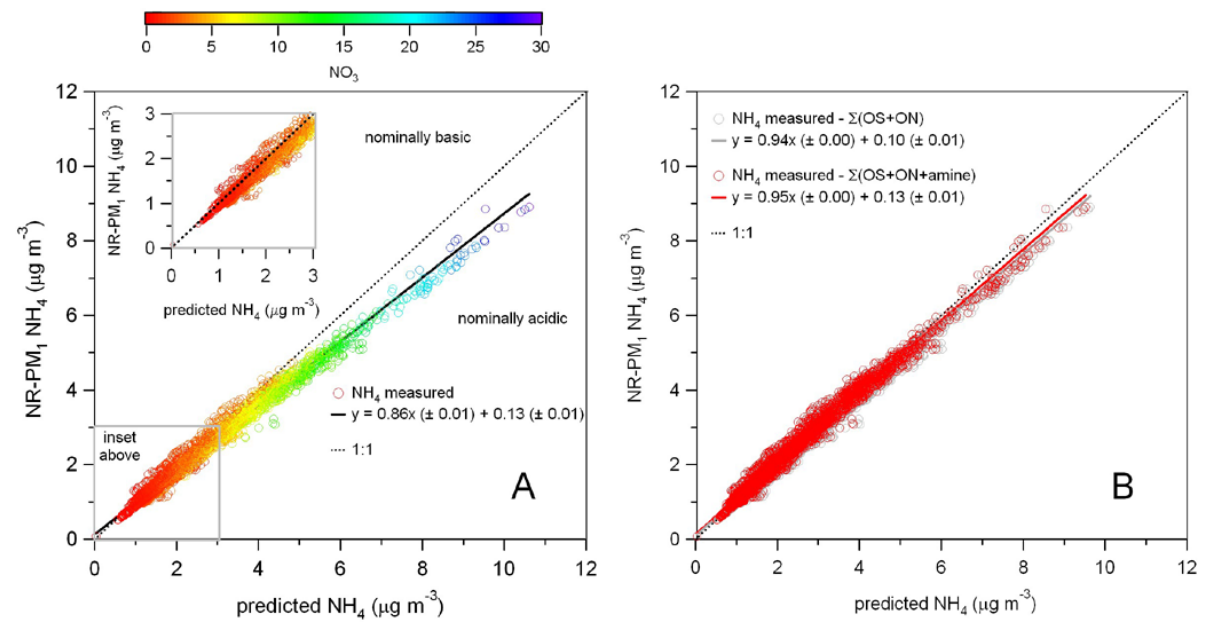

Fig. 12. Anion/cation balance based on HR-AMS measurements. In (a), measured $\mathrm{NH}_{4}$ is plotted against $\mathrm{NH}_{4}$ predicted assuming full neutralization of particulate anions (e.g., $\mathrm{NO}_{3}, \mathrm{SO}_{4}$, and $\mathrm{Cl}$ ). In (b), predicted $\mathrm{NH}_{4}$ has been adjusted to include the contributions from organonitrates, organosulfates, and amine (see text for details).

component TD-AMS-PMF solution contributes $4 \%$ of NR$\mathrm{PM}_{1} \mathrm{OA}$ on average during SOAR-1. We estimate that $17 \%$ of the mass of LOA-AC (i.e. $0.7 \%$ of the OA mass) is accounted by amine functional groups and assume that amines were present as salts which were more strongly associated with $\mathrm{NO}_{3}$ and $\mathrm{SO}_{4}$ during SOAR-1 as indicated by Pratt et al. (2009a). As can be seen by results of linear regression of measured versus predicted $\mathrm{NH}_{4}$ values shown in Fig. $12 \mathrm{~b}$ for each case (i.e., including OS and ON and including OS, ON, and amines), including OS and ON has the largest impact on the HR-AMS ion balance increasing the slope from 0.86 to 0.94 . In contrast, the presence of amines increases the slope only marginally from 0.94 to 0.95 when included in the ion balance calculation.

\subsection{Average $\mathrm{PM}_{2.5}$ composition}

HR-AMS NR-PM 1 measurements and those from an ensemble of $\mathrm{PM}_{2.5}$ instruments were used to separately reconstruct the average $\mathrm{PM}_{2.5}$ composition during SOAR-1 which is shown in Fig. 13. The $\mathrm{PM}_{2.5}$ composition was created using TEOM $_{\mathrm{FDMS}}$, IC-nitrate, and IC-sulfate measurements as well as OC and EC measurements from Sunset1. OC mass was converted to OA mass using hourly $\mathrm{OM} / \mathrm{OC}$ values obtained from EA of HR-AMS OA spectra. Particulate $\mathrm{NH}_{4}$ and non-EC refractory material were not specifically measured but rather were calculated using available data. For example, $\mathrm{NH}_{4}$ was calculated using $\mathrm{PM}_{2.5} \mathrm{NO}_{3}$ and $\mathrm{SO}_{4}$ measurements from the IC-nitrate and IC-sulfate, respectively, assuming full neutralization of these species. Similarly, $\mathrm{PM}_{2.5}$ non-EC refractory material was calculated as the difference between the TEOM ${ }_{\mathrm{FDMS}}$ mass and the sum of $\mathrm{PM}_{2.5}$ species. $\mathrm{PM}_{2.5}$ chloride was also not measured during SOAR-1. However, as discussed above, HR-AMS measurements indicate that NR-PM 1 chloride concentrations are minor and it is therefore likely that the concentration of NR chloride in $\mathrm{PM}_{2.5-1}$ is also very small. Refractory chloride, mostly due to sea salt, can be significantly larger and is absorbed here into non-EC refractory material. Similarly, mass concentrations of $\mathrm{PM}_{2.5}$ non-EC refractory material and NR-PM ${ }_{2.5-1}$ are needed to create the HR-AMS-based ensemble composition. Non-EC refractory material concentrations were determined as above, while NR-PM $2.5-1$ was estimated as the difference between measured TEOM FDMS $_{\text {S }}$ concentrations and the sum of the individual NR-PM $\mathrm{PM}_{1}$ components, Sunset1 EC, and non-EC refractory material. The $\mathrm{PM}_{2.5}$ composition is shown in Fig. 13a while the composition of $\mathrm{PM}_{2.5}$ based on NR-PM $\mathrm{PM}_{1}$ measurements is shown in Fig. 13b. Overall, there is high similarity between both ensemble compositions. Each similarly indicates that $\mathrm{OA}$ is the largest single component of $\mathrm{PM}_{2.5}$ mass. In contrast, the contribution of the major inorganic components $\mathrm{NO}_{3}, \mathrm{SO}_{4}$, and $\mathrm{NH}_{4}$ are slightly higher in the $\mathrm{PM}_{2.5}$ composition. This may be due to inorganic contributions in supermicron particles or other differences in the $\mathrm{SO}_{4}$ or calculated $\mathrm{NH}_{4}$ concentrations as discussed previously. Results from the CalNex study, conducted in the summer of 2010 in Pasadena, also suggest that $\mathrm{OA}$ is largely confined to the submicron mode in the LA basin while the inorganic anions have a supermicron fraction (R. Weber, personal communication, 2010), which is consistent with our results. If the estimates of ON and OS discussed above are correct, this would cause a difference between the NR-PM $\mathrm{PM}_{1}$ and $\mathrm{PM}_{2.5} \mathrm{NO}_{3}, \mathrm{SO}_{4}$ (and the calculated $\mathrm{NH}_{4}$ ) about $10 \%$, since the former would include the signal from $\mathrm{ON}$ and $\mathrm{OS}$ while the latter would not. This would in turn suggest that the fraction of $\mathrm{SO}_{4}$ and $\mathrm{NO}_{3}$ in $\mathrm{PM}_{2.5-1}$ is slightly larger than in the default estimate in Fig. 13a. 
A

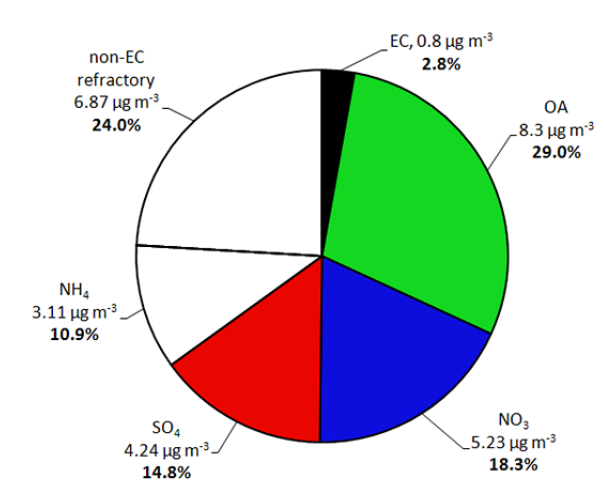

C

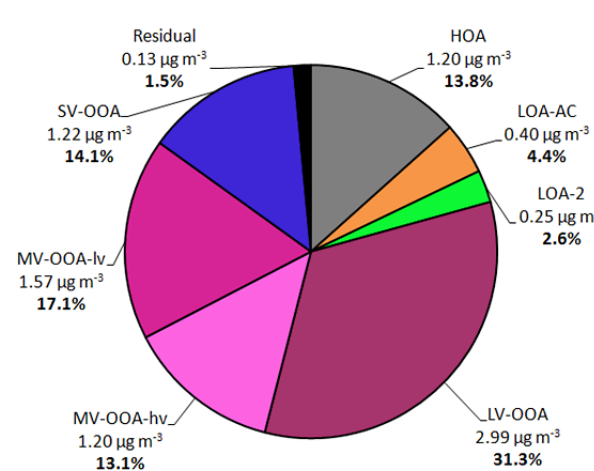

B $\quad \mathrm{PM}_{2.5}$ (based on $\left.\mathrm{PM}_{1}\right)$

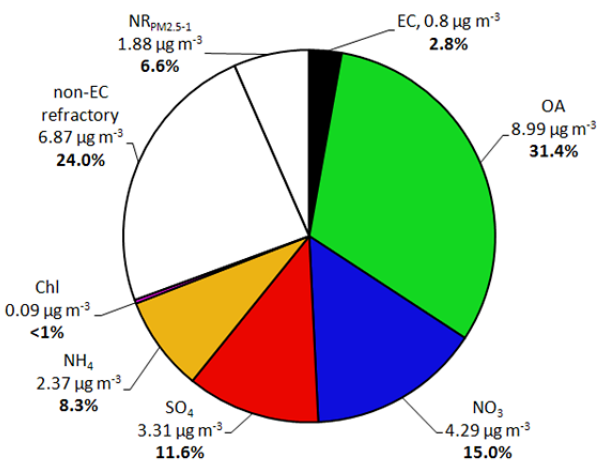

D Results of OA apportionment

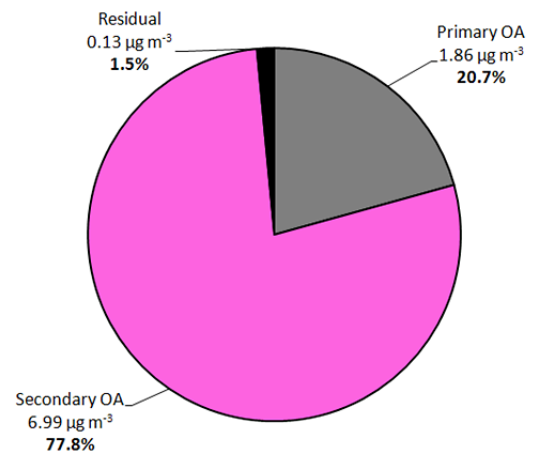

Fig. 13. Average composition of $\mathrm{PM}_{2.5}$ (a-b) and NR-PM 1 OA (c-d) throughout the duration of SOAR-1. (a) shows the average PM 2.5 composition based on $\mathrm{PM}_{2.5}$ measurements while (b) shows the same based on NR-PM 1 measurements. Note that colorless sections (e.g., non-EC refractory material) represent estimated as opposed to measured concentrations. (c) and (d) show the average composition of NR-PM 1 OA and the contribution of POA (composed from the combined HOA, LOA-AC, and LOA-2 components) and SOA (summed contribution of OOA components) to OA during the same period (see text for details).

Finally, the composition of NR-PM 1 OA based on the 7component TD-AMS-PMF solution is summarized in a similar pie chart in Fig. 13c. HOA and the local factors overall have a minor contribution to NR-PM $\mathrm{PM}_{1}$ totaling $20.7 \%$ on average. The remainder of NR-PM 1 OA mass is contributed by the various identified OOA components with the largest single contribution $(31.3 \%)$ coming from the highly oxidized cLV-OOA. Contributions from the remaining OOA components are of the same order and are approximately half of cLV-OOA contributions. If we assume that the sum of HOA and the two LOA components are representative of POA and the sum of OOA components is representative of SOA, SOA contributions are nearly four times larger than POA as shown in Fig. 13d. If MV-OOA-lv is instead considered to be an aged POA component, the SOA is still twice as large as POA.

\section{Conclusions}

Multiple state-of-the-art instruments sampled ambient aerosols during SOAR-1 in order to investigate the sources and composition of $\mathrm{PM}_{f}$ in the inland LA basin. NR-PM measurements from the HR-AMS were consistent with and (for most species) highly correlated to complementary measurements from a suite of $\mathrm{PM}_{2.5}$ instruments. As a result, separate reconstructions of average $\mathrm{PM}_{2.5}$ composition during SOAR-1 were remarkably similar. NR-PM $1+\mathrm{EC}$ accounted for the majority ( $\sim 75 \%$ ) of $\mathrm{PM}_{2.5}$ mass, with nearly a third of NR-PM $\mathrm{PM}_{1}+\mathrm{EC}$ contributed by OA, the single largest component of ambient particles in Riverside. HR-AMS OC is consistent with complementary measurements by available Sunset semi-continuous instruments. Compared to the scatter between the two ToF-AMS instruments, however, the scatter observed between the HR-AMS and Sunset instruments, and between Sunset instruments was substantially higher. The use of a correction designed to account for the presence of SVOC resulted in OC concentrations about $30 \%$ higher than obtained from either the regular Sunset or HR-AMS measurements. Results of elemental analysis of HR-AMS OA indicate that OA in Riverside is slightly less oxidized than in Mexico City. Oxygen is the dominant 
heteroatom in $\mathrm{OA}$, while organic $\mathrm{N}$ and $\mathrm{S}$ are present in at least an-order-of-magnitude lower concentrations. When accounting for the estimated ON and OS concentrations, $\mathrm{H} / \mathrm{C}$ does not change substantially, O/C increases by $21 \%$, N/C increases by a factor of 2 , and $\mathrm{S} / \mathrm{C}$ increases increases by a factor of 24.

The remaining fraction of fine particle mass in Riverside was contributed by inorganics, primarily $\mathrm{NO}_{3}$ and $\mathrm{SO}_{4}$ as their ammonium salts. Diurnal characteristics of each of these components reflect both their different source regions throughout the $\mathrm{SoCAB}$ as well as differences in volatility. $\mathrm{SO}_{4}$ concentrations were relatively stable throughout the day reflecting the consistent presence of $\mathrm{SO}_{4}$ in particles irrespective of changes in the age and origin of air masses thereby highlighting the regional nature of $\mathrm{SO}_{4} . \mathrm{SO}_{4}$ intercomparisons from different instruments had a low degree of correlation potentially due to the presence of a supermicron $\mathrm{SO}_{4}$ fraction. In contrast, particulate $\mathrm{NO}_{3}$ measurements among various instruments were highly correlated and exhibit diurnal profiles different from that of $\mathrm{SO}_{4} . \mathrm{NO}_{3}$ concentrations display two maxima during morning and early afternoon periods and also a profound minimum during the late afternoon concurrent with maximum average daily temperature and minimum humidity. The anions were neutralized by ammonium during SOAR-1 within the uncertainty of this determination. Accounting for the estimated ON and OS improved the quantitative agreement of anions and cations by $\sim 8 \%$, while amines made a very minor impact.

PMF analysis of high resolution HR-AMS OA spectra obtained during SOAR-1 identified several OA components including many having characteristics consistent with corresponding components identified in earlier PMF analyses as well as many that are reported here for the first time. A minor fraction of OA mass $(\sim 20 \%)$ was contributed by directlyemitted and local components. The most significant of these contributions is from HOA, which has been attributed to emissions from mobile sources including diesel and combustion engines. The remainder of the directly-emitted OA mass is contributed by two minor, LOA components. Similar components have been identified in Mexico city (Aiken et al., 2010) and are likely to be unique to individual regions. In this case, the spectrum of LOA-AC shows that this component is strongly associated with amines, which have recently been the subject of increased interest. The characteristics of this component are dissimilar from amine-associated aerosols previously observed in ambient and chamber aerosols and, as a result, we are unable to conclusively identify the chemical identity of this component. However, the widely different volatility characterizations of SOAR-1 amines obtained here and by Pratt et al. (2009a), using the same thermodenuder technique, highlight the need for additional investigations into the chemical identity of similar amine-associated components.
The overwhelming majority $(\sim 80 \%)$ of $\mathrm{NR}-\mathrm{PM}_{1}$ OA mass is contributed by OOA components which are transported into inland regions of the SoCAB from locations closer to the coast and which differ both in the degree to which they are oxidized and their volatility in an inversely proportional relationship. Consistent with earlier PMF analyses of AMS OA spectra, cLV-OOA from SOAR-1 has the highest degree of oxidation and lowest volatility suggesting that this component represents a highly aged aerosol component. SV-OOA lies on the opposite end of this spectrum with a low degree of oxidation and a high volatility. As a result, SV-OOA has been attributed to "fresher" secondary organic aerosols (Jimenez et al., 2009). The spectrum of each of these components (e.g., mass spectrum and correlations with tracer species) is highly similar to corresponding components identified in earlier PMF analyses. Unique to SOAR-1 OA at present, is the identification of two MV-OOA components which have oxidation levels and volatilities intermediate between those of LV-OOA and SV-OOA and may represent a transition between the two as aerosol is aged in the atmosphere. It is worthwhile to note that these two components were successfully separated only in the TD-AMSPMF analysis in which additional variation in the composition of ambient $\mathrm{OA}$ is introduced through the operation of the TD system. The chemical nature of the MV-OOA components and the fate of each are currently unclear at present as is whether similar components exist in other region. Future comparable TD-AMS-PMF analyses are recommended in order to gain greater insight into the sources and fates of these newly identified components.

\section{Supplementary material related to this article is available online at: http://www.atmos-chem-phys.net/11/12387/2011/ acp-11-12387-2011-supplement.pdf.}

Acknowledgements. The research described here was partially supported by EPA STAR grants R831080 and RD-83216101-0, NSF CAREER grant ATM 0449815, NSF/UCAR contract S0539607, by NOAA grant NA08OAR4310565, DOE grants (BER, ASR program) DE-SC0006035 and DE-Fg02-11ER65293 and by CARB award numbers 08-319 and 03-324. ACA, JAH, and IMU acknowledge NASA Earth Science Graduate Fellowships, and PFD acknowledges an EPA STAR Graduate Fellowship. We thank the University of California-Riverside for hosting the site and much logistical support. We would additionally like to thank J. R. Kimmel, M. J. Cubison, E. J. Dunlea, A. Trimborn, and M. Northway for assistance with ToF-AMS operation during SOAR and J. J. Schauer for Sunset1 data.

Edited by: A. Baklanov 


\section{References}

Aiken, A. C., DeCarlo, P. F., and Jimenez, J. L.: Elemental analysis of organic species with electron ionization highresolution mass spectrometry, Anal. Chem., 79, 8350-8358, doi:10.1021/ac071150w, 2007.

Aiken, A. C., DeCarlo, P. F., Kroll, J. H., Worsnop, D. R., Huffman, J. A., Docherty, K. S., Ulbrich, I. M., Mohr, C., Kimmel, J. R., Sueper, D., Sun, Y., Zhang, Q., Trimborn, A., Northway, M., Ziemann, P. J., Canagaratna, M. R., Onasch, T. B., Alfarra, M. R., Prevot, A. S. H., Dommen, J., Duplissy, J., Metzger, A., Baltensperger, U., and Jimenez, J. L.: O/C and OM/OC ratios of primary, secondary, and ambient organic aerosols with high-resolution time-of-flight aerosol mass spectrometry, Environ. Sci. Technol., 42, 4478-4485, doi:10.1021/es703009q, 2008.

Aiken, A. C., Salcedo, D., Cubison, M. J., Huffman, J. A., DeCarlo, P. F., Ulbrich, I. M., Docherty, K. S., Sueper, D., Kimmel, J. R., Worsnop, D. R., Trimborn, A., Northway, M., Stone, E. A., Schauer, J. J., Volkamer, R. M., Fortner, E., de Foy, B., Wang, J., Laskin, A., Shutthanandan, V., Zheng, J., Zhang, R., Gaffney, J., Marley, N. A., Paredes-Miranda, G., Arnott, W. P., Molina, L. T., Sosa, G., and Jimenez, J. L.: Mexico City aerosol analysis during MILAGRO using high resolution aerosol mass spectrometry at the urban supersite (T0) - Part 1: Fine particle composition and organic source apportionment, Atmos. Chem. Phys., 9, 66336653, doi:10.5194/acp-9-6633-2009, 2009.

Aiken, A. C., de Foy, B., Wiedinmyer, C., DeCarlo, P. F., Ulbrich, I. M., Wehrli, M. N., Szidat, S., Prevot, A. S. H., Noda, J., Wacker, L., Volkamer, R., Fortner, E., Wang, J., Laskin, A., Shutthanandan, V., Zheng, J., Zhang, R., Paredes-Miranda, G., Arnott, W. P., Molina, L. T., Sosa, G., Querol, X., and Jimenez, J. L.: Mexico city aerosol analysis during MILAGRO using high resolution aerosol mass spectrometry at the urban supersite (T0) - Part 2: Analysis of the biomass burning contribution and the non-fossil carbon fraction, Atmos. Chem. Phys., 10, 5315-5341, doi:10.5194/acp-10-5315-2010, 2010.

American Lung Association, State of the Air: 2011: http://www. stateoftheair.org/2011/city-rankings/most-polluted-cities.html, last access: 21 November 2011, 2011.

Alfarra, M. R.: Insights into atmospheric organic aerosols using an aerosol mass spectrometer, Ph.D. thesis, University of Manchester, UK, 2004.

Alfarra, M. R., Coe, H., Allan, J. D., Bower, K. N., Boudries, H., Canagaratna, M. R., Jimenez, J. L., Jayne, J. T., Garforth, A. A., Li, S.-M., and Worsnop, D. R.: Characterization of Urban and Rural Organic Particulate in the Lower Fraser Vally using two Aerodyne Aerosol Mass Spectrometers, Atmos. Environ., 38, 5745-5758, 2004.

Allan, J. D., Alfarra, M. R., Bower, K. N., Williams, P. I., Gallagher, M. W., Jimenez, J. L., McDonald, A. G., Nemitz, E., Canagaratna, M. R., Jayne, J. T., Coe, H., and Worsnop, D. R.: Quantitative Sampling Using an Aerodyne Aerosol Mass Spectrometer 2. Measurements of Fine Particulate Chemical Composition in Two U.K. Cities, J. Geophys. Res., 108, 4091-4112, 2003.

Allan, J. D., Delia, A. E., Coe, H., Bower, K. N., Alfarra, M. R., Jimenez, J. L., Middlebrook, A. M., Drewnick, F., Onasch, T. B., Canagaratna, M. R., Jayne, J. T., and Worsnop, D. R.: A generalised method for the extraction of chemically resolved mass spectra from aerodyne aerosol mass spectrometer data, J.
Aerosol. Sci., 35, 909-922, 2004.

Angelino, S., Suess, D. T., and Prather, K. A.: Formation of aerosol particles from reactions of secondary and tertiary alkylamines: Characterization by aerosol time-of-flight mass spectrometry, Environ. Sci. Technol., 35, 3130-3138, 2001.

Appel, B. R., Hoffer, E. M., E.L., K., Wall, S. M., Halk, M., and Knights, R. L.: Analysis of carbonaceous material in Southern California atmospheric aerosols. 2, Environ. Sci. Technol., 13, 98-104, 1979.

Bae, M. S., Schauer, J. J., DeMinter, J. T., Turner, J. R., Smith, D., and Cary, R. A.: Validation of a semi-continuous instrument for elemental carbon and organic carbon using a thermal-optical method, Atmos. Environ., 38, 2885-2893, 2004.

Ban-Weiss, G. A., McLaughlin, J. P., Harley, R. A., M. M., Kirchstetter, T. W., Kean, A. J., Strawa, A. W., Stevenson, E. D., and Kendall, G. R.: Long-term changes in emissions of nitrogen oxides and particulate matter from on-road gasoline and diesel vehicles, Atmos. Environ., 42, 220-232, 2008.

Barsanti, K. C., McMurry, P. H., and Smith, J. N.: The potential contribution of organic salts to new particle growth, Atmos. Chem. Phys., 9, 2949-2957, doi:10.5194/acp-9-2949-2009, 2009.

Canagaratna, M. R., Jayne, J. T., Ghertner, D. A., Herndon, S., Shi, Q., Jimenez, J. L., Silva, P. J., Williams, P., Lanni, T., Drewnick, F., Demerjian, K. L., Kolb, C. E., and Worsnop, D. R.: Chase Studies of Particulate Emissions fron In-Use New York City Vehicles, Aerosol Sci. Technol., 38, 555-573, 2004.

Canagaratna, M., Jayne, J., Jimenez, J., Allan, J., Alfarra, M., Zhang, Q., Onasch, T., Drewnick, F., Coe, H., Middlebrook, A., Delia, A., Williams, L., Trimborn, A., Northway, M., DeCarlo, P., Kolb, C., Davidovits, P., and Worsnop, D.: Chemical and microphysical characterization of ambient aerosols with the Aerodyne Aerosol Mass Spectrometer, Mass Spec. Rev., 26, 185-222, 2007.

California Air Resources Board, Emissions Inventory Data: http:// www.arb.ca.gov/app/emsinv/trends/ems_trends.php, last access: 21 September 2011, 2009.

Cheng, Y., He, K. B., Duan, F. K., Zheng, M., Ma, Y. L., and Tan, J. H.: Measurement of semivolatile carbonaceous aerosols and its implications: A review, Environ. Int., 35, 674-681, doi:10.1016/j.envint.2008.11.007, 2009.

Chhabra, P. S., Flagan, R. C., and Seinfeld, J. H.: Elemental analysis of chamber organic aerosol using an aerodyne high-resolution aerosol mass spectrometer, Atmos. Chem. Phys., 10, 4111-4131, doi:10.5194/acp-10-4111-2010, 2010.

Chow, J. C., Watson, J. G., Fujita, E. M., Lu, Z. Q., Lawson, D. R., and Ashbaugh, L. L.: Temporal and spatial variations of $\mathrm{PM}_{2.5}$ and $\mathrm{PM}_{10}$ in the Southern California air quality study, Atmos. Environ., 28, 2061-2080, 1994.

Christoforou, C. S., Salmon, L. G., Hannigan, M. P., Solomon, P. A., and Cass, G. R.: Trends in fine particle concentration and chemical composition in Southern California, J. Air Waste Manage. Assoc., 50, 43-53, 2000.

Cubison, M. J., Ervens, B., Feingold, G., Docherty, K. S., Ulbrich, I. M., Shields, L., Prather, K., Hering, S., and Jimenez, J. L.: The influence of chemical composition and mixing state of Los Angeles urban aerosol on CCN number and cloud properties, Atmos. Chem. Phys., 8, 5649-5667, doi:10.5194/acp-8-5649-2008, 2008. 
Day, D. A., Liu, S., Russell, L. M., and Ziemann, P. J.: Organonitrate group concentrations in submicron particles with high nitrate and organic fractions in coastal southern California, Atmos. Environ., 44, 1970-1979, doi:10.1016/j.atmosenv.2010.02.045, 2010.

DeCarlo, P. F., Kimmel, J. R., Trimborn, A., Northway, M. J., Jayne, J. T., Aiken, A. C., Gonin, M., Fuhrer, K., Horvath, T., Docherty, K. S., Worsnop, D. R., and Jimenez, J. L.: Field-deployable, high-resolution, time-of-flight aerosol mass spectrometer, Anal. Chem., 78, 8281-8289, 2006.

DeCarlo, P. F., Ulbrich, I. M., Crounse, J., de Foy, B., Dunlea, E. J., Aiken, A. C., Knapp, D., Weinheimer, A. J., Campos, T., Wennberg, P. O., and Jimenez, J. L.: Investigation of the sources and processing of organic aerosol over the Central Mexican Plateau from aircraft measurements during MILAGRO, Atmos. Chem. Phys., 10, 5257-5280, doi:10.5194/acp-10-52572010, 2010.

de Gouw, J. and Jimenez, J. L.: Organic aerosols in Earth's atmosphere, Environ. Sci. Technol., 43, 7614-7618, 2009.

Denkenberger, K. A., Moffet, R. C., Holecek, J. C., Rebotier, T. P., and Prather, K. A.: Real-time, single-particle measurements of oligomers in aged ambient aerosol particles, Environ. Sci. Technol., 41, 5439-5446, doi:10.1021/es0703291, 2007.

Docherty, K. S. and Jimenez, J. L.: Study of Organic Aerosols at Riverside (SOAR) 2005: http://cires.colorado.edu/ jimenez-group/Field/Riverside05/, last access: 15 September 2010, 2005.

Docherty, K. S., Stone, E. A., Ulbrich, I. M., DeCarlo, P. F., Snyder, D. C., Schauer, J. J., Peltier, R. E., Weber, R. J., Murphy, S. N., Seinfeld, J. H., Grover, B. D., Eatough, D. J., and Jiimenez, J. L.: Apportionment of Primary and Secondary Organic Aerosols in Southern California during the 2005 Study of Organic Aerosols in Riverside (SOAR-1), Environ. Sci. Technol., 42, 7655-7662, doi:10.1021/es8008166, 2008.

Drewnick, F., Hings, S. S., DeCarlo, P., Jayne, J. T., Gonin, M., Fuhrer, K., Weimer, S., Jimenez, J. L., Demerjian, K. L., Borrmann, S., and Worsnop, D. R.: A new time-of-flight aerosol mass spectrometer (TOF-AMS) - Instrument description and first field deployment, Aerosol Sci. Technol., 39, 637-658, doi:10.1080/02786820500182040, 2005.

Eatough, D. J., Long, R. W., Modey, W. K., and Eatough, N. L.: Semi-volatile secondary organic aerosol in urban atmospheres: meeting a measurement challenge, Atmos. Environ., 37, 12771292, doi:10.1016/s1352-2310(02)01020-8, 2003.

Eatough, D. J., Grover, B. D., Woolwine, W. R., Eatough, N. L., Prather, K. A., Shields, L., Qin, X., Denkenberger, K., Long, R., and Farber, R.: Source apportionment of $1 \mathrm{hr}$ semi-continuous data during the 2005 Study of Organic Aerosols in Riverside (SOAR) using positive matrix factorization, Atmos. Environ., 42, 2706-2719, doi:10.1016/j.atmosenv.2007.07.038, 2008.

Eckerle, E., Ghasemi, A., Hsaio, K., and Yan, S.: Final 2007 air quality management plan - Appendix III - Base and future year emission inventories, South Coast Air Quality Management District, Diamond Bar, CA, 206 pp., 2007.

Erupe, M. E., Price, D. J., Silva, P. J., Malloy, Q. G. J., Qi, L., Warren, B., and Cocker III, D. R.: Secondary organic aerosol formation from reaction of tertiary amines with nitrate radical, Atmos. Chem. Phys. Discuss., 8, 16585-16608, doi:10.5194/acpd8-16585-2008, 2008.
Facchini, M. C., Decesari, S., Rinaldi, M., Carbone, C., Finessi, E., Mircea, M., Fuzzi, S., Moretti, F., Tagliavini, E., Ceburnis, D., and O'Dowd, C. D.: Important Source of Marine Secondary Organic Aerosol from Biogenic Amines, Environ. Sci. Technol., 42, 9116-9121, doi:10.1021/es8018385, 2008.

Farmer, D. K., Matsunaga, A., Docherty, K. S., Surratt, J. D., Seinfeld, J. H., Ziemann, P. J., and Jimenez, J. L.: Response of an aerosol mass spectrometer to organonitrates and organosulfates and implications for atmospheric chemistry, Proc. Natl. Acad. Sci. U.S.A., 107, 6670-6675, doi:10.1073/pnas.0912340107, 2010.

Favez, O., Cachier, H., Sciare, J., and Le Moullec, Y.: Characterization and contribution to $\mathrm{PM}_{2.5}$ of semi-volatile aerosols in Paris (France), Atmos. Environ., 41, 7969-7976, doi:10.1016/j.atmosenv.2007.09.031, 2007.

Fine, P. M., Jaques, P. A., Hering, S. V., and Sioutas, C.: Performance evaluation and use of a continuous monitor for measuring size-fractionated $\mathrm{PM}_{2.5}$ nitrate, Aerosol Sci. Technol., 37, 342354, doi:10.1080/02786820390125188, 2003.

Fraser, M. P., Yue, Z. W., and Buzcu, B.: Source Apportionment of Fine Particulate Matter in Houston, TX, Using Organic Molecular Markers, Atmos. Environ., 37, 2117-2123, 2003.

Fry, J. L., Kiendler-Scharr, A., Rollins, A. W., Wooldridge, P. J., Brown, S. S., Fuchs, H., Dubé, W., Mensah, A., dal Maso, M., Tillmann, R., Dorn, H.-P., Brauers, T., and Cohen, R. C.: Organic nitrate and secondary organic aerosol yield from $\mathrm{NO}_{3}$ oxidation of $\beta$-pinene evaluated using a gas-phase kinetics/aerosol partitioning model, Atmos. Chem. Phys., 9, 14311449, doi:10.5194/acp-9-1431-2009, 2009.

Gao, S., Keywood, M., Ng, N. L., Surratt, J., Varutbangkul, V., Bahreini, R., Flagan, R. C., and Seinfeld, J. H.: LowMolecular Weight and Oligomeric Components in Secondary Organic Aerosol from the Ozonolysis of Cycloalkenes and alphapinene, J. Phys. Chem. A, 108, 10147-10164, 2004.

Ge, X., Wexler, A. S., and Clegg, S. L.: Atmospheric amines - Part I, A review, Atmos. Environ., 45, 524-546, 2011.

Gray, H. A., Cass, G. R., Huntzicker, J. J., Heyerdahl, E. K., and Rau, J. A.: Characteristics of atmospheric organic and elemental carbon particle concentrations in Los Angeles, Environ. Sci. Technol., 20, 580-589, 1986.

Grover, B. D., Kleinman, M., Eatough, N. L., Eatough, D. J., Hopke, P. K., Long, R. W., Wilson, W. E., Meyer, M. B., and Ambs, J. L.: Measurement of total $\mathrm{PM}_{2.5}$ mass (nonvolatile plus semivolatile) with the Filter Dynamic Measurement System tapered element oscillating microbalance monitor, J. Geophys. Res.-Atmos., 110, D07S03, doi:10.1029/2004JD004995, 2005.

Grover, B. D., Eatough, N. L., Eatough, D. J., Chow, J. C., Watson, J. G., Ambs, J. L., Meyer, M. B., Hopke, P. K., AlHorr, R., Later, D. W., and Wilson, W. E.: Measurement of both nonvolatile and semi-volatile fractions of fine particulate matter in Fresno, CA, Aerosol Sci. Technol., 40, 811-826, doi:10.1080/02786820600615071, 2006.

Grover, B. D., Eatough, N. L., Woolwine, W. R., Cannon, J. P., Eatough, D. J., and Long, R. W.: Semi-continuous mass closure of the major components of fine particulate matter in Riverside, CA, Atmos. Environ., 42, 250-260, doi:10.1016/j.atmosenv.2007.09.037, 2008.

Grover, B. D., Eatough, N. L., Woolwine, W. R., Eatough, D. J., and Cary, R. A.: Modifications to the Sunset Laboratory 
Carbon Aerosol Monitor for the Simultaneous Measurement of $\mathrm{PM}_{2.5}$ Nonvolatile and Semi-Volatile Carbonaceous Material, J. Air Waste Manage. Assoc., 59, 1007-1017, doi:10.3155/10473289.59.8.1007, 2009.

Hallquist, M., Wenger, J. C., Baltensperger, U., Rudich, Y., Simpson, D., Claeys, M., Dommen, J., Donahue, N. M., George, C., Goldstein, A. H., Hamilton, J. F., Herrmann, H., Hoffmann, T., Iinuma, Y., Jang, M., Jenkin, M. E., Jimenez, J. L., Kiendler-Scharr, A., Maenhaut, W., McFiggans, G., Mentel, Th. F., Monod, A., Prévôt, A. S. H., Seinfeld, J. H., Surratt, J. D., Szmigielski, R., and Wildt, J.: The formation, properties and impact of secondary organic aerosol: current and emerging issues, Atmos. Chem. Phys., 9, 5155-5236, doi:10.5194/acp-95155-2009, 2009.

Heald, C. L., Kroll, J. H., Jimenez, J. L., Docherty, K. S., DeCarlo, P. F., Aiken, A. C., Chen, Q., Martin, S. T., Farmer, D. K., and Artaxo, P.: A simplified description of the evolution of organic aerosol composition in the atmosphere, Geophys. Res. Lett., 37, 5, L08803, doi:10.1029/2010gl042737, 2010.

Herndon, S. C., Onasch, T. B., Wood, E. C., Kroll, J. H., Canagaratna, M. R., Jayne, J. T., Zavala, M. A., Knighton, W. B., Mazzoleni, C., Dubey, M. K., Ulbrich, I. M., Jimenez, J. L., Seila, R. de Gouw, J. A., de Foy, B., Fast, J., Molina, L. T., Kold, C. E., Worsnop, D. R.: The correlation of secondary organic aerosol with odd oxygen in a Mexico City, Geophys. Res. Lett., 35, L15804, doi: 10.1029/2008GL034058, 2008.

Hildemann, L. M., Cass, G. R., Mazurek, M. A., and Simoneit, B. R. T.: Mathematical modeling of urban organic aerosol - properties measured by high-resolution gas chromatography, Environ. Sci. Technol., 27, 2045-2055, 1993.

Hings, S. S., Walter, S., Schneider, J., Borrmann, S., and Drewnick, F.: Comparison of a quadrupole and a time-of-flight aerosol mass spectrometer during the Feldberg aerosol characterization experiment 2004, Aerosol Sci. Technol., 41, 679-691, doi:10.1080/02786820701408483, 2007.

Huang, M., Carmichael, G. R., Spak, S. N., Adhikary, B., Kulkarni, S., Cheng, Y., Wei, C., Tang, Y., D’Allura, A., Wennberg, P. O., Huey, G. L., Dibb, J. E., Jimenez, J. L., Cubison, M. J., Weinheimer, A. J., Kaduwela, A., Cai, C., Wong, M., Bradley Pierce, R., Al-Saadi, J. A., Streets, D. G., and Zhang, Q.: Multi-scale modeling study of the source contributions to nearsurface ozone and sulfur oxides levels over California during the ARCTAS-CARB period, Atmos. Chem. Phys., 11, 3173-3194, doi:10.5194/acp-11-3173-2011, 2011.

Huffman, J. A., Ziemann, P. J., Jayne, J. T., Worsnop, D. R., and Jimenez, J. L.: Development and characterization of a fast-stepping/scanning thermodenuder for chemically-resolved aerosol volatility measurements, Aerosol Sci. Technol., 42, 395407, doi:10.1080/02786820802104981, 2008.

Huffman, J. A., Docherty, K. S., Aiken, A. C., Cubison, M. J., U1brich, I. M., DeCarlo, P. F., Sueper, D., Jayne, J. T., Worsnop, D. R., Ziemann, P. J., and Jimenez, J. L.: Chemically-resolved aerosol volatility measurements from two megacity field studies, Atmos. Chem. Phys., 9, 7161-7182, doi:10.5194/acp-9-71612009, 2009.

Iida, K., Stolzenburg, M. R., McMurry, P. H., Smith, J. N., Quant, F. R., Oberreit, D. R., Keady, P. B., Eiguren-Fernandez, A., Lewis, G. S., Kreisberg, N. M., and Hering, S. V.: An ultrafine, water-based condensation particle counter and its evalua- tion under field conditions, Aerosol Sci. Technol., 42, 862-871, doi:10.1080/02786820802339579, 2008.

Jang, M. and McDow, S. R.: Products of benz[a]anthracene photodegradation in the presence of known organic constituents of atmospheric aerosols, Environ. Sci. Technol., 31, 1046-1053, 1997.

Jayne, J. T., Leard, D. C., Zhang, Z., Davidovits, P., Smith, K. A., Kolb, C. E., and Worsnop, D. R.: Development of an Aerosol Mass Spectrometer for Size and Composition Analysis of Submicron Particles, Aerosol Sci. Technol., 33, 49-70, 2000.

Jimenez, J. L., Jayne, J. T., Shi, Q., Kolb, C. E., Worsnop, D. R., Yourshaw, I., Seinfeld, J. H., Flagan, R. C., Zhang, X. F., Smith, K. A., Morris, J. W., and Davidovits, P.: Ambient aerosol sampling using the Aerodyne Aerosol Mass Spectrometer, J. Geophys. Res.-Atmos., 108, D7, 8425, doi:10.1029/2001 JD001213, 2003.

Jimenez, J. L., Canagaratna, M. R., Donahue, N. M., Prevot, A. S. H., Zhang, Q., Kroll, J. H., DeCarlo, P. F., Allan, J. D., Coe, H., Ng, N. L., Aiken, A. C., Docherty, K. S., Ulbrich, I. M., Grieshop, A. P., Robinson, A. L., Duplissy, J., Smith, J. D., Wilson, K. R., Lanz, V. A., Hueglin, C., Sun, Y. L., Tian, J., Laaksonen, A., Raatikainen, T., Rautiainen, J., Vaattovaara, P., Ehn, M., Kulmala, M., Tomlinson, J. M., Collins, D. R., Cubison, M J., Dunlea, E. J., Huffman, J. A., Onasch, T. B., Alfarra, M. R., Williams, P. I., Bower, K., Kondo, Y., Schneider, J., Drewnick, F., Borrmann, S., Weimer, S., Demerjian, K., Salcedo, D., Cottrell, L., Griffin, R., Takami, A., Miyoshi, T., Hatakeyama, S., Shimono, A., Sun, J. Y., Zhang, Y. M., Dzepina, K., Kimmel, J. R., Sueper, D., Jayne, J. T., Herndon, S. C., Trimborn, A. M., Williams, L. R., Wood, E. C., Middlebrook, A. M., Kolb, C. E., Baltensperger, U., and Worsnop, D. R.: Evolution of Organic Aerosols in the Atmosphere, Science, 326, 1525-1529, doi:10.1126/science.1180353, 2009.

Kalberer, M., Paulson, D., Sax, M., Steinbacher, M., Dommen, J., Prèvôt, A. S. H., Fissiha, R., Weingartner, E., Frankevich, V., Zenobi, R., and Baltensperger, U.: Identification of Polymers as Major Components of Atmospheric Organic Aerosols, Science, 303, 1659-1662, 2004.

Kean, A. J., Harley, R. A., Littlejohn, D., and Kendall, G. R.: Onroad measurement of ammonia and other motor vehicle exhaust emissions, Environ. Sci. Technol., 34, 3535-3539, 2000.

Kim, B. M., Teffera, S., and Zeldin, M. D.: Characterization of $\mathrm{PM}_{2.5}$ and $\mathrm{PM}_{10}$ in the South Coast Air Basin of southern California: Part 1 - Spatial variations, J. Air Waste Manage. Assoc., 50, 2034-2044, 2000.

Kim, E. and Hopke, P. K.: Source characterization of ambient fine particles in the Los Angeles basin, J. Environ. Engin. Sci., 6, 343-353, doi:10.1139/s06-054, 2007.

Kleindienst, T. E., Jaoui, M., Lewandowski, M., Offenberg, J. H., Lewis, C. W., Bhave, P. V., and Edney, E. O.: Estimates of the contributions of biogenic and anthropogenic hydrocarbons to secondary organic aerosol at a southeastern US location, Atmos. Environ., 41, 8288-8300, doi:10.1016/j.atmosenv.2007.06.045, 2007.

Kondo, Y., Miyazaki, Y., Takegawa, N., Miyakawa, T., Weber, R. J., Jimenez, J. L., Zhang, Q., and Worsnop, D. R.: Oxygenated and water-soluble organic aerosols in Tokyo, J. Geophys. Res.Atmos., 112, D01203, doi:10.1029/2006JD007056 , 2007.

Kreisberg, N. M., Hering, S. V., Williams, B. J., Worton, 
D. R., and Goldstein, A. H.: Quantification of Hourly Speciated Organic Compounds in Atmospheric Aerosols, Measured by an In-Situ Thermal Desorption Aerosol Gas Chromatograph (TAG), Aerosol Sci. Technol., 43, 38-52, doi:10.1080/02786820802459583, 2009.

Lanz, V. A., Alfarra, M. R., Baltensperger, U., Buchmann, B., Hueglin, C., and Prévôt, A. S. H.: Source apportionment of submicron organic aerosols at an urban site by factor analytical modelling of aerosol mass spectra, Atmos. Chem. Phys., 7, 1503-1522, doi:10.5194/acp-7-1503-2007, 2007.

Lee, T., Sullivan, A. P., Mack, L., Jimenez, J. L., Kreidenweis, S. M., Onasch, T., Worsnop, D. R., Malm, W., Wold, C. E., Hao, W. M., and Collett, J. L.: Chemical smoke marker emissions during flaming and smoldering phases of laboratory open burning of wildland fuels, Aerosol Sci. Technol., 44, i-v, doi:10.1080/02786826.2010.299884, 2010.

Lester, J. C. and Woods, M.: Final staff report: Proposed Rule 1127 - Emissions reductions from livestock waste, South Coast Air Quality Management District, Diamond Bar, CA, 67 pp., 2004. Liu, P. S. K., Deng, R., Smith, K. A., Williams, L. R., Jayne, J. T., Canagaratna, M. R., Moore, K., Onasch, T. B., Worsnop, D. R., and Deshler, T.: Transmission efficiency of an aerodynamic focusing lens system: Comparison of model calculations and laboratory measurements for the Aerodyne Aerosol Mass Spectrometer, Aerosol Sci. Technol., 41, 721-733, doi:10.1080/02786820701422278, 2007.

Lloyd, J. A., Heaton, K. J., and Johnston, M. V.: Reactive Uptake of Trimethylamine into Ammonium Nitrate Particles, J. Phys. Chem. A , 113, 4840-4843, doi:10.1021/jp900634d, 2009.

Lukács, H., Gelencsér, A., Hoffer, A., Kiss, G., Horvth, K., and Hartyni, Z.: Quantitative assessment of organosulfates in sizesegregated rural fine aerosol, Atmos. Chem. Phys., 9, 231-238, doi:10.5194/acp-9-231-2009, 2009.

Malloy, Q. G. J., Li Qi, Warren, B., Cocker III, D. R., Erupe, M. E., and Silva, P. J.: Secondary organic aerosol formation from primary aliphatic amines with $\mathrm{NO}_{3}$ radical, Atmos. Chem. Phys., 9, 2051-2060, doi:10.5194/acp-9-2051-2009, 2009.

Matsunaga, A., Docherty, K. S., Lim, Y. B., and Ziemann, P. J.: Composition and yields of secondary organic aerosol formed from $\mathrm{OH}$ radical-initiated reactions of linear alkenes in the presence of $\mathrm{NO}_{\mathrm{x}}$ : Modeling and measurements, Atmos. Environ., 43, 1349-1357, doi:10.1016/j.atmosenv.2008.12.004, 2009.

McLafferty, F. W. and Turecek, F.: Interpretation of Mass Spectra, 4th edn., University Science Books, Sausalito, CA, 1993.

Middlebrook, A. M., Bahreini, R., Jimenez, J. L., Canagaratna, M. R.,: Evaluation of composition-dependent collection efficiencies for the Aerodyne aerosol mass spectormeter using field data, Aerosol Sci. Technol., 46, 258-271, doi:10.1080/02786826.2011.620041, 2011.

Mohr, C., Huffman, J. A., Cubison, M. J., Aiken, A. C., Docherty, K. S., Kimmel, J. R., Ulbrich, I. M., Hannigan, M., and Jimenez, J. L.: Characterization of Primary Organic Aerosol Emissions from Meat Cooking, Trash Burning, and Motor Vehicles with High-Resolution Aerosol Mass Spectrometry and Comparison with Ambient and Chamber Observations, Environ. Sci. Technol., 43, 2443-2449, doi:10.1021/es8011518, 2009.

Muller, C., Iinuma, Y., Karstensen, J., van Pinxteren, D., Lehmann, S., Gnauk, T., and Herrmann, H.: Seasonal variation of aliphatic amines in marine sub-micrometer particles at the Cape Verde is- lands, Atmos. Chem. Phys., 9, 9587-9597, 2009,

http://www.atmos-chem-phys.net/9/9587/2009/.

Murphy, S. M., Sorooshian, A., Kroll, J. H., Ng, N. L., Chhabra, P., Tong, C., Surratt, J. D., Knipping, E., Flagan, R. C., and Seinfeld, J. H.: Secondary aerosol formation from atmospheric reactions of aliphatic amines, Atmos. Chem. Phys., 7, 2313-2337, doi:10.5194/acp-7-2313-2007, 2007.

Neuman, J. A., Nowak, J. B., Brock, C. A., Trainer, M., Fehsenfeld, F. C., Holloway, J. S., Hubler, G., Hudson, P. K., Murphy, D. M., Nicks, D. K., Orsini, D., Parrish, D. D., Ryerson, T. B., Sueper, D. T., Sullivan, A., and Weber, R.: Variability in ammonium nitrate formation and nitric acid depletion with altitude and location over California, J. Geophys. Res.-Atmos., 108, D17, 4557, doi:10.1029/2003jd003616, 2003.

Ng, N. L., Canagaratna, M. R., Zhang, Q., Jimenez, J. L., Tian, J., Ulbrich, I. M., Kroll, J. H., Docherty, K. S., Chhabra, P. S., Bahreini, R., Murphy, S. M., Seinfeld, J. H., Hildebrandt, L., Donahue, N. M., DeCarlo, P. F., Lanz, V. A., Prévôt, A. S. H., Dinar, E., Rudich, Y., and Worsnop, D. R.: Organic aerosol components observed in Northern Hemispheric datasets from Aerosol Mass Spectrometry, Atmos. Chem. Phys., 10, 46254641, doi:10.5194/acp-10-4625-2010, 2010.

Ng, N. L., Canagaratna, M. R., Jimenez, J. L., Chhabra, P. S., Seinfeld, J. H., and Worsnop, D. R.: Changes in organic aerosol composition with aging inferred from aerosol mass spectra, Atmos. Chem. Phys. Discuss., 11, 7095-7112, doi:10.5194/acpd11-7095-2011, 2011.

Paatero, P. and Hopke, P. K.: Discarding or downweighting highnoise variables in factor analytic models, Anal. Chim. Acta, 490, 277-289, 2003.

Paatero, P. and Tapper, U.: Positive matrix factorization - a nonnegative factor model with optimal utilization of error estimates of data values, Environmetrics, 5, 111-126, 1994.

Pandis, S. N., Harley, R. A., Cass, G. R., and Seinfeld, J. H.: Secondary organic aerosol formation and transport, Atmos. Environ. A-Gen, 26, 2269-2282, 1992.

Paredes-Miranda, G., Arnott, W. P., Jimenez, J. L., Aiken, A. C., Gaffney, J. S., and Marley, N. A.: Primary and secondary contributions to aerosol light scattering and absorption in Mexico City during the MILAGRO 2006 campaign, Atmos. Chem. Phys., 9, 3721-3730, doi:10.5194/acp-9-3721-2009, 2009.

Park, K., Chow, J. C., Watson, J. G., Trimble, D. L., Doraiswamy, P., Arnott, W. P., Stroud, K. R., Bowers, K., Bode, R., Petzold, A., and Hansen, A. D. A.: Comparison of continuous and filterbased carbon measurements at the Fresno Supersite, J. Air Waste Manage. Assoc., 56, 474-491, 2006.

Patashnik, H., Rupprecht, G., Ambs, J. L., and Meyer, M. B.: Development of a reference standard for particular matter mass in ambient air, Aerosol Sci. Technol., 34, 42-45, 2001.

Peltier, R. E., Weber, R. J., and Sullivan, A. P.: Investigating a liquid-based method for online organic carbon detection in atmospheric particles, Aerosol Sci. Technol., 41, 1117-1127, 2007.

Phuleria, H. C., Fine, P. M., Zhu, Y. F., and Sioutas, C.: Air quality impacts of the October 2003 Southern California wildfires, J. Geophys. Res.-Atmos., 110, 11, D07S20, doi:10.1029/2004JD004626, 2005.

Pratt, K. A. and Prather, K. A.: Real-time, single-particle volatility, size, and chemical composition measurements of aged urgan aerosols, Environ. Sci. Technol., 43, 8276-8282, 2009. 
Pratt, K. A., Hatch, L. E., and Prather, K. A.: Seasonal Volatility Dependence of Ambient Particle Phase Amines, Environ. Sci. Technol., 43, 5276-5281, doi:10.1021/es803189n, 2009a.

Pratt, K. A., Mayer, J. E., Holecek, J. C., Moffet, R. C., Sanchez, R. O., Rebotier, T. P., Furutani, H., Gonin, M., Fuhrer, K., Su, Y. X., Guazzotti, S., and Prather, K. A.: Development and Characterization of an Aircraft Aerosol Time-of-Flight Mass Spectrometer, Anal. Chem., 81, 1792-1800, doi:10.1021/ac801942r, 2009 b.

Qin, X., Pratt, K. A., Shields, L. G., Toner, S. M., and Prather, K. A.: Seasonal comparisons of the single particle mixing state in Riverside, CA, Atmos. Environ., submitted, 2011.

Reemtsma, T., These, A., Venkatachari, P., Xia, X. Y., Hopke, P. K., Springer, A., and Linscheid, M.: Identification of fulvic acids and sulfated and nitrated analogues in atmospheric aerosol by electrospray ionization Fourier transform ion cyclotron resonance mass spectrometry, Anal. Chem., 78, 82998304, doi:10.1021/ac061320p, 2006.

Salcedo, D., Dzepina, K., Onasch, T. B., Canagaratna, M. R., Zhang, Q., Huffman, A.. R., DeCarlo, P. F., Jayne, J. T., Mortimer, P., Worsnop, D. R., Kolb, C. E., Johnson, K. S., Zuberi, B., Marr, L. C., Molina, L. T., Molina, M. J., Bernab, R. M., Cardenas, B., Mrquez, C., Gaffney, J. S., Marley, N. A., Laskin, A., Shutthanandan, V., and Jimenez, J. L.: Characterization of ambient aerosols in Mexico City during the MCMA-2003 campaign with Aerosol Mass Spectrometry - Part I: quantification, shape-related collection efficiency, and comparison with collocated instruments, Atmos. Chem. Phys. Discuss., 5, 4143-4182, doi:10.5194/acpd-5-4143-2005, 2005.

Salcedo, D., Onasch, T. B., Dzepina, K., Canagaratna, M. R., Zhang, Q., Huffman, J. A., DeCarlo, P. F., Jayne, J. T., Mortimer, P., Worsnop, D. R., Kolb, C. E., Johnson, K. S., Zuberi, B., Marr, L. C., Volkamer, R., Molina, L. T., Molina, M. J., Cardenas, B., Bernab, R. M., Mrquez, C., Gaffney, J. S., Marley, N. A., Laskin, A., Shutthanandan, V., Xie, Y., Brune, W., Lesher, R., Shirley, T., and Jimenez, J. L.: Characterization of ambient aerosols in Mexico City during the MCMA-2003 campaign with Aerosol Mass Spectrometry: results from the CENICA Supersite, Atmos. Chem. Phys., 6, 925-946, doi:10.5194/acp-6-9252006, 2006.

Schade, G. W. and Crutzen, P. J.: Emissions of aliphatic amines from animal husbandry and their reactions - potential source of $\mathrm{N}_{2} \mathrm{O}$ and HCN, J. Atmos. Chem., 22, 319-346, 1995.

Schauer, J. J., Rogge, W. F., Hildemann, L. M., Mazurek, M. A., and Cass, G. R.: Source apportionment of airborne particulate matter using organic compounds as tracers, Atmos. Environ., 30, 3837-3855, 1996.

Schauer, J. J., Fraser, M. P., Cass, G. R., and Simoneit, B. R. T.: Source reconciliation of atmospheric gas-phase and particlephase pollutants during a severe photochemical smog episode, Environ. Sci. Technol., 36, 3806-3814, 2002.

Schauer, J. J., Fraser, M. P., Cass, G. R., Simoneit, B. R. T.: Source reconciliation of atmospheric gas-phase and particle-phase pollutants during a severe photochemical smog episode, Environ. Sci. Technol., 36, 3806-3814, 2002.

Schauer, J. J., Mader, B. T., Deminter, J. T., Heidemann, G., Bae, M. S., Seinfeld, J. H., Flagan, R. C., Cary, R. A., Smith, D., Huebert, B. J., Bertram, T., Howell, S., Kline, J. T., Quinn, P., Bates, T., Turpin, B., Lim, H. J., Yu, J. Z., Yang, H., and Keywood, M. D.: ACE-Asia intercomparison of a thermal-optical method for the determination of particle-phase organic and elemental carbon, Environ. Sci. Technol., 37, 993-1001, 2003.

Schwab, J. J., Felton, H. D., Rattigan, O. V., and Demerjian, K. L.: New York state urban and rural measurements of continuous $\mathrm{PM}_{2.5}$ mass by FDMS, TEOM, and BAM, J. Air Waste Manage. Assoc., 56, 372-383, 2006.

Shilling, J. E., Chen, Q., King, S. M., Rosenoern, T., Kroll, J. H., Worsnop, D. R., DeCarlo, P. F., Aiken, A. C., Sueper, D., Jimenez, J. L., and Martin, S. T.: Loading-dependent elemental composition of a-pinene SOA particles, Atmos. Chem. Phys., 9, 771-782, doi:10.5194/acp-9-771-2009, 2009.

Silva, P. J., Erupe, M. E., Price, D., Elias, J., Malloy, Q. G. J., Li, Q., Warren, B., and Cocker, D. R.: Trimethylamine as precursor to secondary organic aerosol formation via nitrate radical reaction in the atmosphere, Environ. Sci. Technol., 42, 4689-4696, doi:10.1021/es703016v, 2008.

Smith, J. N., Barsanti, K. C., Friedli, H. R., Ehn, M., Kulmala, M., Collins, D. R., Scheckman, J. H., Williams, B. J., and McMurry, P. H.: Observations of aminium salts in atmospheric nanoparticles and possible climatic implications, Proc. Natl. Acad. Sci. U.S.A., 107, 6634-6639, doi:10.1073/pnas.0912127107, 2010.

Snyder, D. C. and Schauer, J. J.: An inter-comparison of two black carbon aerosol instruments and a semi-continuous elemental carbon instrument in the urban environment, Aerosol Sci. Technol., 41, 463-474, doi:10.1080/02786820701222819, 2007.

Snyder, D. C., Dallmann, T. R., Schauer, J. J., Holloway, T., Kleeman, M. J., Geller, M. D., and Sioutas, C.: Direct observation of the break-up of a nocturnal inversion layer using elemental mercury as a tracer, Geophys. Res. Lett., 35, 5, L17812, doi:10.1029/2008gl034840, 2008.

Sorooshian, A., Murphy, S. M., Hersey, S., Gates, H., Padro, L. T., Nenes, A., Brechtel, F. J., Jonsson, H., Flagan, R. C., and Seinfeld, J. H.: Comprehensive airborne characterization of aerosol from a major bovine source, Atmos. Chem. Phys., 8, 5489-5520, doi:10.5194/acp-8-5489-2008, 2008.

South Coast Air Quality Management District, Traffic and Vehicle Data: http://www.aqmd.gov/smog/o3trend.html, last access: 15 September 2010.

Stolzenburg, M. R. and Hering, S. V.: Method for the automated measurement of fine particle nitrate in the atmosphere, Environ. Sci. Technol., 34, 907-914, 2000.

Stone, E. A., Zhou, J. B., Snyder, D. C., Rutter, A. P., Mieritz, M., and Schauer, J. J.: A Comparison of Summertime Secondary Organic Aerosol Source Contributions at Contrasting Urban Locations, Environ. Sci. Technol., 43, 3448-3454, doi:10.1021/es8025209, 2009.

Sueper, D.: ToF-AMS Analysis Software - available online at: http://cires.colorado.edu/jimenez-group/wiki/index.php/ ToF-AMS __\}Analysis __\}Software, 2009.

Sun, Y.-L., Zhang, Q., Schwab, J. J., Demerjian, K. L., Chen, W.N., Bae, M.-S., Hung, H.-M., Hogrefe, O., Frank, B., Rattigan, O. V., and Lin, Y.-C.: Characterization of the sources and processes of organic and inorganic aerosols in New York city with a high-resolution time-of-flight aerosol mass spectrometer, Atmos. Chem. Phys., 11, 1581-1602, doi:10.5194/acp-11-15812011, 2011.

Surratt, J. D., Kroll, J. H., Kleindienst, T. E., Edney, E. O., Claeys, M., Sorooshian, A., Ng, N. L., Offenberg, J. H., Lewandowski, M., Jaoui, M., Flagan, R. C., and Seinfeld, J. H.: Evidence 
for organosulfates in secondary organic aerosol, Environ. Sci. Technol., 41, 517-527, 2007.

Surratt, J. D., Gomez-Gonzalez, Y., Chan, A. W. H., Vermeylen, R., Shahgholi, M., Kleindienst, T. E., Edney, E. O., Offenberg, J. H., Lewandowski, M., Jaoui, M., Maenhaut, W., Claeys, M., Flagan, R. C., and Seinfeld, J. H.: Organosulfate formation in biogenic secondary organic aerosol, J. Phys. Chem. A, 112, 8345-8378, doi:10.1021/jp802310p, 2008.

Takegawa, N., Miyazaki, Y., Kondo, Y., Komazaki, Y., Miyakawa, T., Jimenez, J. L., Jayne, J. T., Worsnop, D. R., Allan, J. D., and Weber, R. J.: Characterization of an Aerodyne Aerosol Mass Spectrometer (AMS): Intercomparison with other aerosol instruments, Aerosol Sci. Technol., 39, 760-770, 2005.

Tolocka, M. P., Jang, M., Ginter, J. M., Cox, F. J., Kamens, R. M., and Johnston, M. V.: Formation of Oligimers in Secondary Organic Aerosol, Environ. Sci. Technol., 38, 1428-1434, 2004.

Turpin, B. J. and Huntzicker, J. J.: Identification of secondary organic aerosol episodes and quantitation of primary and secondary organic aerosol concentrations during SCAQS, Atmos. Environ., 29, 3527-3544, 1995.

Turpin, B. J. and Lim, H. J.: Species contributions to $\mathrm{PM}_{2.5}$ mass concentrations: Revisiting common assumptions for estimating organic mass, Aerosol Sci. Technol., 35, 602-610, 2001.

Ulbrich, I. M., Canagaratna, M. R., Zhang, Q., Worsnop, D. R., and Jimenez, J. L.: Interpretation of organic components from Positive Matrix Factorization of aerosol mass spectrometric data, Atmos. Chem. Phys., 9, 2891-2918, doi:10.5194/acp-9-2891-2009, 2009.

Vutukuru, S. and Dabdub, D.: Modeling the effects of ship emissions on coastal air quality: A case study of southern California, Atmos. Environ., 42, 3751-3764, doi:10.1016/j.atmosenv.2007.12.073, 2008.

Wang, L., Lal, V., Khalizov, A. F., and Zhang, R. Y.: Heterogeneous Chemistry of Alkylamines with Sulfuric Acid: Implications for Atmospheric Formation of Alkylaminium Sulfates, Environ. Sci. Technol., 44, 2461-2465, doi:10.1021/es9036868, 2010.

Weber, R. J., Sullivan, A. P., Peltier, R. E., Russell, A., Yan, B., Zheng, M., de Gouw, J., Warneke, C., Brock, C., Holloway, J. S., Atlas, E. L., and Edgerton, E.: A study of secondary organic aerosol formation in the anthropogenic-influenced southeastern United States, J. Geophys. Res.-Atmos., 112, D13302, doi:10.1029/2004JD008408, 2007.

Westerholm, R., Li, H., and Almen, J.: Estimation of aliphatic amine emissions in automobile exhausts, Chemosphere, 27, 1381-1384, 1993.
Williams, B. J., Goldstein, A. H., Kreisberg, N. M., and Hering, S. V.: An in-situ instrument for speciated organic composition of atmospheric aerosols: Thermal Desorption Aerosol GC/MS-FID (TAG), Aerosol Sci. Technol., 40, 627-638, 2006.

Williams, B. J., Goldstein, A. H., Kreisberg, N. M., Hering, S. V., Worsnop, D. R., Ulbrich, I. M., Docherty, K. S., and Jimenez, J. L.: Major components of atmospheric organic aerosol in southern California as determined by hourly measurements of source marker compounds, Atmos. Chem. Phys. Discuss., 10, 65676639, doi:10.5194/acpd-10-6567-2010, 2010.

Wilson, W. E., Grover, B. D., Long, R. W., Eatough, N. L., and Eatough, D. J.: The measurement of fine-particulate semivolatile material in urban aerosols, J. Air Waste Manage. Assoc., 56, 384-397, 2006.

Zhang, Q., Alfarra, M. R., Worsnop, D. R., Allan, J. D., Coe, H., Canagaratna, M. R., and Jimenez, J. L.: Deconvolution and quantification of hydrocarbon-like and oxygenated organic aerosols based on aerosol mass spectrometry, Environ. Sci. Technol., 39, 4938-4952, 2005a.

Zhang, Q., Canagaratna, M. R., Jayne, J. T., Worsnop, D. R., and Jimenez, J. L.: Time- and size-resolved chemical composition of submicron particles in Pittsburgh: Implications for aerosol sources and processes, J. Geophys. Res.-Atmos., 110, 19, D07S09, doi:10.1029/2004JD004649, 2005b.

Zhang, Q., Jimenez, J. L., Canagaratna, M. R., Allan, J. D., Coe, H., Ulbrich, I., Alfarra, M. R., Takami, A., Middlebrook, A. M., Sun, Y. L., Dzepina, K., Dunlea, E., Docherty, K., DeCarlo, P. F., Salcedo, D., Onasch, T., Jayne, J. T., Miyoshi, T., Shimono, A., Hatakeyama, S., Takegawa, N., Kondo, Y., Schneider, J., Drewnick, F., Borrmann, S., Weimer, S., Demerjian, K., Williams, P., Bower, K., Bahreini, R., Cottrell, L., Griffin, R. J., Rautiainen, J., Sun, J. Y., Zhang, Y. M., and Worsnop, D. R.: Ubiquity and dominance of oxygenated species in organic aerosols in anthropogenically-influenced Northern Hemisphere midlatitudes, Geophys. Res. Lett., 34, L13801, doi:10.1029/2007GL029979, 2007.

Zheng, M., Cass, G. R., Schauer, J. J., and Edgerton, E. S.: Source apportionment of PM2.5 in the southeastern United States using solvent-extractable organic compounds as tracers, Environ. Sci. Technol., 36, 2361-2371, 2002. 